\title{
Versicherungs-Statistik für 1903
}

\section{Einleitung.}

Durch die vorliegende Statistik soll der Vorschrift des $\$ 83$ des Versicherungsaufsichtsgesetzes, wonach das Kaiserliche Aufsichtsamt jährlich Mitteilungen über den Stand der seiner Aufsicht unterliegenden Versicherungsunternehmungen zu veröffentlichen hat, für das Jahr 1903, das zweite der Aufsichtsführung des Amtes, genügt werden.

Unter der Aufsicht des Kaiserlichen Aufsichtsamts stehen 1. diejenigen inländischen privaten Versicherungsunternehmungen, deren Geschäftsbetrieb nicht durch die Satzung oder der Reichi die sonstigen Geschäftsunterlagen auf das Gebiet eines Bundesstaats beschränkt ist (§2 des aufsicht Versicherungsaufsichtsgesetzes), soweit sie nicht etwa bei sachlich, örtlich oder hinsichtlich des Personenkreises eng begrenzter Ausdehnung vom Reichskanzler im Einvernehmen mit den beteiligten Landesbehörden der Aufsicht einer Landesbehörde überwiesen worden sind (§ 3 Abs. 2 a. a. O.), 2. diejenigen inländischen privaten Unternehmungen, deren Geschäftsbetrieb auf das Gebiet eines Bundesstaats beschränkt ist, deren Beaufsichtigung aber auf Antrag dieses Bundesstaats mit Zustimmung des Bundesrats durch Kaiserliche Verordnung dem Aufsichtsamt übertragen ist (§3 Abs. 1 a. a. O.), 3. die ausländischen Versicherungsunternehmungen, die im Inlande durch Vertreter, Bevollmächtigte, Agenten oder sonstige Vermittler das Versicherungsgeschäft betreiben ( $\$ 91$ a. a. 0. ).

Ausgenommen von der Reichsaufsicht sind 1. Unternehmungen, welche die Versicherung gegen Kursverluste oder die Transportversicherung oder ausschließlich die Rückversicherung zum Gegenstande haben, mit Ausnahme von Versicherungsvereinen auf Gegenseitigkeit (§116 a. a. O.), 2. die eingeschriebenen Hülfskassen, die auf Grund landesrechtlicher Vorschriften errichteten Hülfskassen, die auf Grund der Gewerbeordnung von Innungen oder Innungsverbänden errichteten Unterstützungskassen sowie die auf Grund berggesetzlicher Vorschriften errichteten Knappschaftskassen (§ 122 a. a. 0.).

I)er hierdurch umgrenzte Teil des privaten Versicherungswesens im Deutschen Reiche Der Beob ist in dieser Statistik, soweit das auf Grund der dem Aufsichtsamte für das Jahr $\mathrm{r}_{903}{ }_{\text {sohtungs. }}^{\text {stoff }}$ zugegangenen Berichte der Unternehmungen möglich ist, ausführlich dargestellt. Grundsätzlich ausgeschlossen sind nur diejenigen Unternehmungen, deren Beaufsichtigung auf Grund des erwähnten $\$ 3$ Abs. 1 dem Aufsichtsamt überwiesen ist; es waren das im Jahre ${ }_{1903}$ Versicherungsunternehmungen, die ihren Sitz im Großherzogtum Hessen, im Fürstentume Schaumburg-Lippe und in der freien Hansestadt Bremen haben und ihren Geschäftsbetrieb über ihren Heimatstaat nicht hinauserstrecken. Ihre Zahl ist zwar nicht klein, dennoch aber fallen sie ihrer ganzen Bedeutung nach nicht ins Gewicht; ihre statistische Bearbeitung wird sich erst empfehlen, wenn eine entsprechende Arbeit für alle oder doch für die größeren Bundesstaaten eingeleitet wird. 
Das in den Tabellen zusammengestellte Zahlenmaterial beruht auf den Jahresabschlüssen, die dem Amte gemäß seinen „Vorschriften über die Rechnungslegung der vom Kaiserlichen Aufsichtsamte für Privatversicherung beaufsichtigten größeren Versicherungsunternehmungen" (Veröffentlichungen des Amtes 1902 S. 23 ff.) eingereicht werden. Diese Vor'schriften, die je besonders für Lebensversicherungsunternehmungen, für Unfall- und Haftpflichtversicherungsunternehmungen, für Hagel- und Viehversicherungsunternehmungen und für Feuerversicherungsunternehmungen erlassen worden sind, hatten zuerst für das nach dem 31. Dezember r 90 I beginnende Geschäftsjahr Anwendung zu finden; demgemäß ist bei Unternehmungen, deren Geschäftsjahr nicht mit dem Kalenderjahre zusammenfällt, in dieser Statistik dasjenige Geschäftsjahr zu Grunde gelegt, dessen Beginn in das Jahr 1903 fällt.

In der Einleitung zu der vorjährigen Statistik ist darauf hingewiesen worden, daß der damals verwendete Beobachtungsstoff nicht überall die wünschenswerte Vollkommenheit besaß. Die Nachprüfung der Abschlüsse für das Geschäftsjahr 1903 hat denn auch noch eine ganze Reihe von Zahlen der vorjährigen Abschlüsse als unrichtig erwiesen, die bei der Bearbeitung der Statistik für 1902 für richtig gehalten werden mußten. Diese Zahlen sind in den Anmerkungen zu den Tabellen für 1903 gekennzeichnet und außerdem unter den Berichtigungen am Schlusse dieses Bandes aufgeführt.

Die sämtlichen Unternehmungen sind nach der Natur ihres Betriebs hier in dieselben fünf Gruppen eingeteilt, auf welche gemäß § 8 der Kaiserlichen Verordnung, betreffend das Verfahren und den Geschäftsgang des Kaiserlichen Aufsichtsamts für Privatversicherung, vom 23. Dezember 190 r die Mitglieder des Versicherungsbeirats verteilt sind, nämlich:

1. Lebensversicherung und Krankenversicherung,

2. Unfall- und Haftpflichtversicherung,

3. Viehversicherung, Hagelversicherung und sonstige landwirtschaftliche Versicherung,

4. Feuerversicherung, Versicherung gegen Sturmschäden, Wasserschäden und Diebstahl,

5. Sonstige Versicherungszweige.

Innerhalb dieser Gruppen sind die Unternehmungen nach den von ihnen betriebenen Versicherungszweigen getrennt, folgen sich aber in allen Tabellen in derselben Reihe und stets unter derselben Nummer.

\section{Allgemeiner Überblick.}

Die Unter- Diese Statistik berichtet eingehend über 199 deutsche und 54 ausländische Unter-

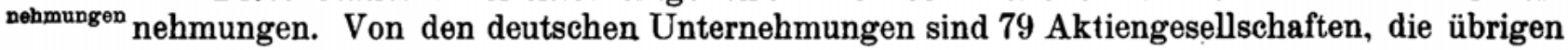
beruhen, abgesehen von wenigen Einzelunternehmern und Genossenschaften mit beschränkter Haftpflicht, auf dem Grundsatze der Gegenseitigkeit.

Während von den Gegenseitigkeitsvereinen sich nur 5 gleichzeitig in verschiedenen .Versicherungszweigen betätigten, betrieben von den 79 Aktiengesellschaften 43 deren mehrere. Namentlich die kleineren Versicherungszweige: Die Sturmschäden-, die Wasserleitungsschäden-, die Einbruchdiebstahlversicherung, die Glas-, die Kautions-, Garantieund Kreditversicherung sowie die Maschinenversicherung, aber auch die Unfall- und Haftpflichtversicherung werden fast ausschließlich von Aktiengesellschaften gepflegt. Die Maschinenversicherung, der jüngste Sproß auf den Versicherungsgebiete, ist erst in dem Berichtsjahr und zwar von nur einer Gesellschaft in Betrieb genommen worden; sie will die Schäden ersetzen, welche an Maschinen und maschinellen Einrichtungen entstehen 
infolge von Unfällen durch den Betrieb, infolge Ungeschicklichkeit, Fahrlässigkeit oder Böswilligkeit der Arbeiter oder anderer Personen, oder durch Sturm, Wolkenbruch oder Eisgang, infolge von Kurzschluß oder endlich bei Montage oder Demontage innerhalb des Betriebsgrundstücks. Es darf hier vorgreifend bemerkt werden, daß sich im Jahre r904 noch zwei weitere Aktiengesellschaften der Maschinenversicherung zugewandt haben.

Von den in dieser Statistik ausführlich behandelten deutschen Unternehmungen haben im Berichtsjahre gearbeitet in der Lebensversicherung 28 Aktiengesellschaften und 38 Gegenseitigkeitsvereine, in der Unfallversicherung 29 Aktiengesellschaften und 1 Gegenseitigkeitsverein, in der Haftpflichtversicherung 25 Aktiengesellschaften und 1 Verein, in der Viehversicherung nur Gegenseitigkeitsvereine und zwar 24, in der Hagelversicherung 5 Aktiengesellschaften (sämtlich mit Ausschluß anderer Versicherungsgeschäfte) und 11 Gegenseitigkeitsvereine (drei von diesen betrieben auch die Feuerversicherung und einer die Glasversicherung ', in der Feuerversicherung 33 Aktiengesellschaften und 16 Vereine, in der Versicherung gegen Einbruchdiebstahl 32 Aktiengesellschaften, und endlich in der Glasversicherung 19 Aktiengesellschaften und 4 Vereine. Von 31 Kaskoversicherungsunternehmungen sei in diesem Überblick nur erwähnt, daß bei ihnen am Schlusse des Berichtsjahrs reichlich 7000 Fahrzeuge im Werte von 45,79 Millionen Mark versichert waren.

Von den 54 ausländischen zum Geschäftsbetrieb im Deutschen Reiche befugten Unternehmungen - solche mit weniger als 100000 , Jl Jahresprämieneinnahme sind bei der Sachversicherung nicht berücksichtigt worden - hatten 3 in den Vereinigten Staaten von Amerika ihren Hauptsitz, 3 in Belgien, 3 in Dänemark, 15 in England, 3 in Frankreich, 5 in den Niederlanden, 11 in Österreich, 2 in Schweden und 9 in der Schweiz. Gearbeitet haben von ihnen 22 in der Lebensversicherung, 7 in der Unfall- und Haftpflichtversicherung, 25 in der Feuerversicherung, 9 in der Einbruchdiebstahl- und 5 in der Glasversicherung. Die geringfügigen Kautions- und Garantieversicherungsgeschäfte, die von 2 dieser Gesellschaften abgeschlossen sind, haben keine weitere Behandlung gefunden.

Der Lmfang des Versicherungsgeschäfts der deutschen Unternehmungen soll durch die folgenden Übersichten in großen Zügen veranschaulicht werden. Dabei ist die Lebensversicherung von den anderen Zweigen getrennt, einmal, weil es zweckmäBig erschien, hier die Nebenarten für sich darzustellen, dann auch, um schon äußerlich daran zu erinnern, daß Versicherungssumme in der Lebensversicherung einen anderen Sinn hat als in der Sachversicherung. In der Lebensversicherung ist es derjenige Wert, der beim Eintritt des Versicherungsfalls voll zahlbar werden soll, in der Sachversicherung aber ist es meistens der Höchstwert, der bei völliger V'ernichtung des versicherten Gegenstandes fällig werden kann.

Umfang des Geschäfts der deutschen Versicherungsunternehmungen

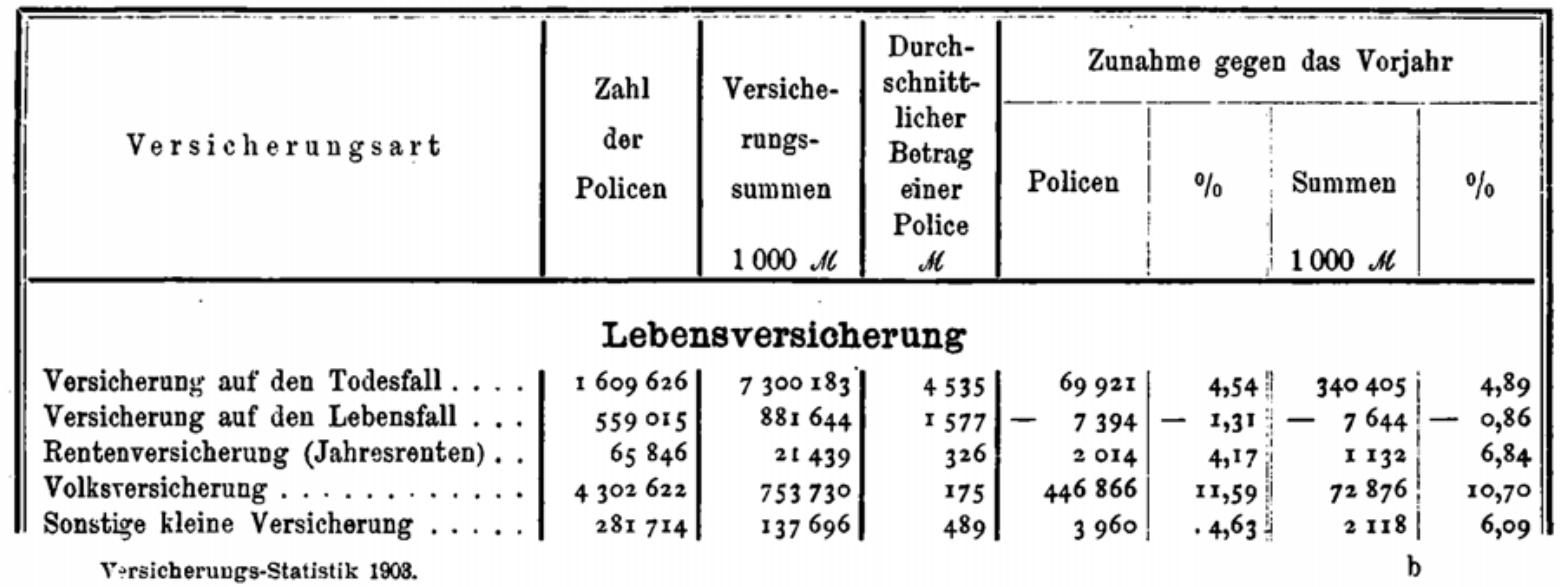


In dem Berichtsjahre sind von den Versicherten an Prämien und Policegebühren an die Unternehmungen gezahlt worden: in der großen Lebensversicherung einschl. der "sonstigen kleinen Versicherung" 341,16 Millionen Mark und in der Volksversicherung 56,32 Millionen, in demselben Jahre sind für eingetretene Versicherungsfälle und für vorzeitig aufgelöste Versicherungen in der großen Versicherung nebst der sonstigen kleinen Versicherung 181,04 Millionen Mark und in der Volksversichernng 8,75 Millionen Mark bar an die Versicherten zurückgeflossen und außerdem 158,3s und 28,46 Millionen Mark den Prämienreserven zugeführt worden. Die Prämien der Versicherten sind aber nicht mit den Gegenleistungen der Unternehmungen in ein und demselben Jahre vergleichbar, weil in der Lebensversicherung einerseits dio Jahresprämien nur zum Teil für die Versicherungsleistungen des Jahres verwendet werden dürfen, zum Teil als Prämienreserve für künftige Versicherungsleistungen zurückgestellt werden müssen, und andererseits der Nettozuwachs der Prämienreserven nicht nur durch die Entnahmen für Versicherungsleistungen und die Überweisungen aus der Prämieneinnahme sondern auch durch Zinseneinnahmen bedingt wird. Daß die baren Auszahlungen in der Volksversicherung so niedrig sind, hat seinen Hauptgrund darin, daß die Volksversicherung bei uns erst im letzten Jahrzehnt zu großer Entwickelung gekommen ist, und mithin vorläufig noch keine nennenswerten Beträge fällig werden können.

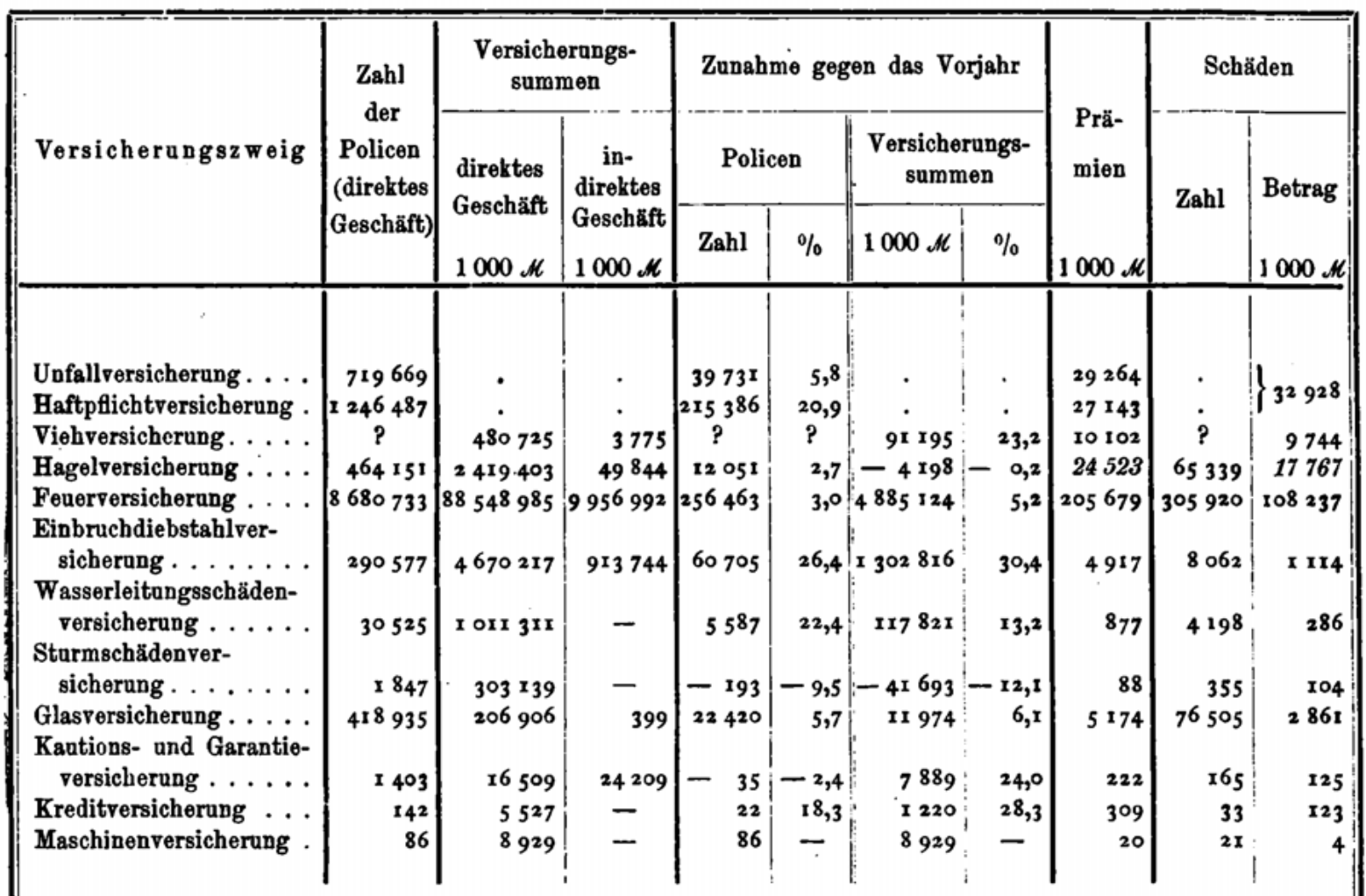

Dio Prämien und Schäden verstehen sich einschließlich der Anteile der Rückversicherer, nur bei der Hagelversicherung sind diese Anteile ausgeschlossen.

Diese Zahlen, die einer Erläuterung wohl nicht bedürfen, mögen noch durch folgende ergänzt werden. Von dem gesamten bei den deutschen Lebensversicherungsgesellschaften Ende 1903 versicherten Kapital (9073,25 Millionen Mark) kommen 8519,55 Millionen Mark auf das deutsche Geschäft, dagegen hatten die ausländischen Lebensversicherungsgesellschaften zu derselben Zeit im Deutschen Reiche 781,25 Millionen Mark versichert, ihr Anteil an dem gesamten deutschen Kapitalversicherungsgeschäfte (9300,so Millionen Mark) stellt sich danach auf 8,4 Prozent. 
Beurteilt man in der Unfall- und Haftpflichtversicherung den Umfang des Geschäfts nach der Jahrespränieneinnahme, so kommen von dem mit 56,41 Millionen Mark zu bewertenden gesamten Geschäfte der deutschen Gesellschaften 49,87 Millionen Mark auf das deutsche Geschäft, die ausländischen Gesellschaften haben aus ihrem deutschen Geschäfte 10,96 Millionen Mark eingenommen, sie sind also an dem gesamten deutschen Geschäfte (60,83 Millionen Mark) mit 18,0 Prozent beteiligt gewesen.

Von den 88549 Millionen Mark, welche die deutschen Unternehmungen Ende 1903 gegen Feuersgefahr versichert hatten, kamen 85327 Millionen auf ihr deutsches Geschäft, wogegen die ausländischen Gesellschaften 11418 Millionen Mark versichert hatten, auf sie entfielen danach 11,8 Prozent von dem gesamten deutschen Geschäfte (96745 Millionen Mark).

Die folgende Übersicht soll einen Einblick in die finanziellen Ergebnisse des Berichtsjahrs und in den Vermögensstand an Schlusse des Geschäftsjahrs für die Gesamtheit der deutschen Unternehmungen mit Ausnahme der Kaskoversicherungsvereine gewähren.

Deutsche Unternehmungen - Gesamtgeschäft

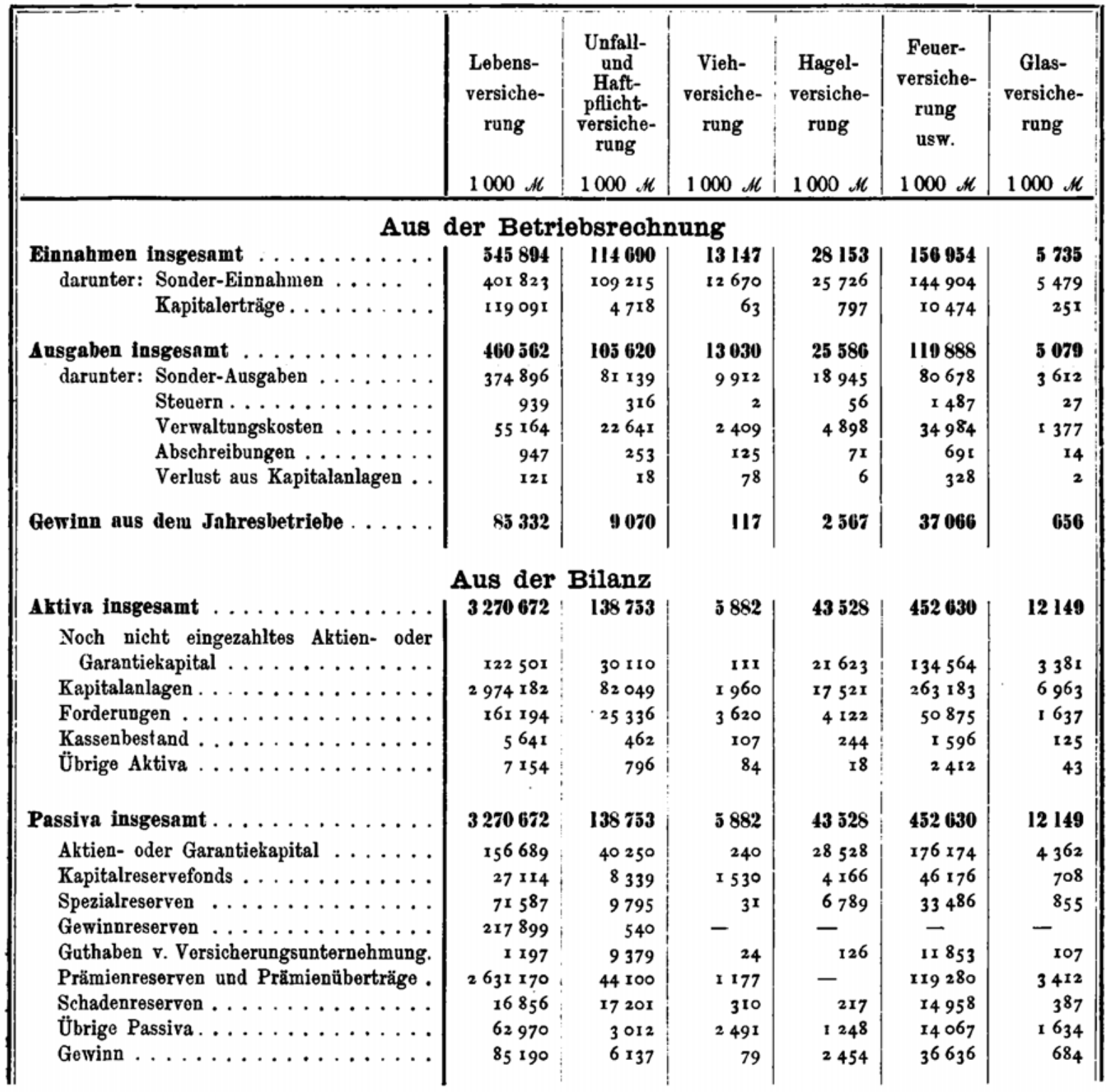


Hierzu ist folgendes zu bemerken. Es ist schon erwähnt, daß die meisten Aktiengesellschaften mehrere Versicherungszweige betreiben. Die Gesellschaften dieser Art trennen dann entweder die verschiedenen Geschäftszweige in allen Posten der Einnahmen und Ausgaben und geben für jeden Zweig eine besondere Übersicht über den Betrieb, oder sie trennen nur bestimmte Posten nach Zweigen und führen andere wie Kapitalerträge und Verwaltungskosten als gemeinsame Kosten aller Zweige. Im ersteren Falle ist in den vorstehenden Angaben über die Einnahmen und Ausgaben die Gesellschaft bei jedem Versicherungszweige mit demjenigen Teile ihrer Einnahmen und Ausgaben berücksichtigt, der auf den betreffenden Zweig entfällt; im zweiten Falle dagegen, wo in der Betriebsrechnung nur eine teilweise Trennung der Posten vorgenommen wird, ist die Gesellschaft mit den Einnahmen und Ausgaben aller ihrer Geschäftszweige nur bei einer Gruppe, nämlich bei derjenigen aufgeführt, welcher der Hauptzweig des Geschäfts - von der Transportversicherung abgesehen, weil sie dem Versicherungsaufsichtsgesetze nicht untersteht - einzuordnen ist. - Eine Bilanz wird dagegen immer nur für das Gesamtgeschäft aufgestellt und bei dem Hauptversicherungszweige der Gesellschaft nachgewiesen. Die Angaben über die Einnahmen und Ausgaben sind also in der vorstehenden Übersicht schärfer nach Versicherungszweigen zerlegt als die über die Aktiva und Passiva. Ferner sind die unter den Einnahmen und Ausgaben mitgeteilten Zahlen das Ergebnis lediglich des Betriebs des Geschäftsjahrs, während in der Bilanz auch der Gewinn- oder Verlustvortrag aus dem Vorjahr eingeschlossen ist (bei einer Sterbekasse z. B., die aus dem Berichtsjahr einen Gewinn von $1 / 8$ Million erzielt hat, war aus den Vorjahren ein Verlust von 4,6 Millionen Mark vorzutragen). Darin hat es seinen Grund, daß in der Betriebsrechnung ein anderer Gewinn erscheint wie in der Bilanz.

In der Verschiedenheit der Rechnungslegung, die sich bei den Gesellschaften unit gemischtem Betriebe findet, ist auch die ZweckmäBigkeit begründet, gewisse den einzelnen Geschäftszweigen eigentümliche Einnahme- und Ausgabeposten als Sonder-Einnahmen und Sonder-Ausgaben zusammenzufassen. Als Sonder-Einnahmen sind verrechnet die Prämien, die Nebenleistungen der Versicherten (Policegebühren, Eintrittsgelder usw.), die Ersparnis aus der Schadenreserve, bei der Viehversicherung auch der Erlös aus verwertetem Vieh, als Sonder-Ausgaben die Zahlungen für Versicherungsfälle und für vorzeitige Auflösungen von Versicherungen, die Regulierungskosten sowie ein etwaiger Zuschuß zur Schadenreserve, bei der Lebens- sowie der Unfall- und Haftpflichtversicherung auch die Zunahme der Prämienreserve. Bei diesen beiden Zweigen verstehen sich die Zahlen einschließlich des Anteils der Rückversicherer, bei den andern ohne diesen.

In der Bilanz stehen 79 Aktiengesellschaften mit 401,66 Millionen Mark Aktienkapital, wovon 93,01 Millionen Mark eingezahlt sind, und 89 Gegenseitigkeitsvereine mit einem Garantiekapitale von 4,58 Millionen, mit 0,94 Millionen Einzahlung. Die für die Aktiengesellschaften in $\S 262$ des Handelsgesetzbuchs und für die Gegenseitigkeitsvereine in $\S 37$ des Versicherungsaufsichtsgesetzes vorgeschriebenen Kapitalreservefonds beliefen sich Ende r 1903 für alle Unternehmungen zusammen auf 88,03 Millionen; dazu kamen 122,54 Millionen Mark Spezialreserven, die freilich zum Teil nicht als freie Reserven betrachtet werden können. Die Prämienreserven und die Prämienüberträge, 2 799,14 Millionen, meist auch die Gewinnreserven, 218,44 Millionen, sowie die Schadenreserven, 49,93 Millionen, sind als gebunden zu betrachten, da sie so gut wie vollständig zur Deckung bereits entstandener Ansprüche der Versicherten dienen.

Die Kapitalanlagen aller dieser 168 Unternehmungen haben Ende rgo3 insgesamt 3 345,s6 Millionen Mark betragen, davon entfallen 96,12 Millionen auf Grundbesitz, 2714,4o Millionen auf Hypotheken und Grundschulden, 3,97 Millionen auf Darlehen auf Wertpapiere, 315,92 Millionen auf Wertpapiere, 200,60 Millionen auf Policedarlehen, 8,,0 Millionen auf Wechsel und 6,30 Millionen auf sonstige Anlagen. Die Verteilung auf diese Wertarten 
ist aber bei den verschiedenen Versicherungszweigen und zum Teil auch bei den beiden Gesellschaftsformen eine recht verschiedene.

Von 1000 ،// Kapitalanlagen kommen nämlich bei den deutschen Versicherungsunternehmungen auf

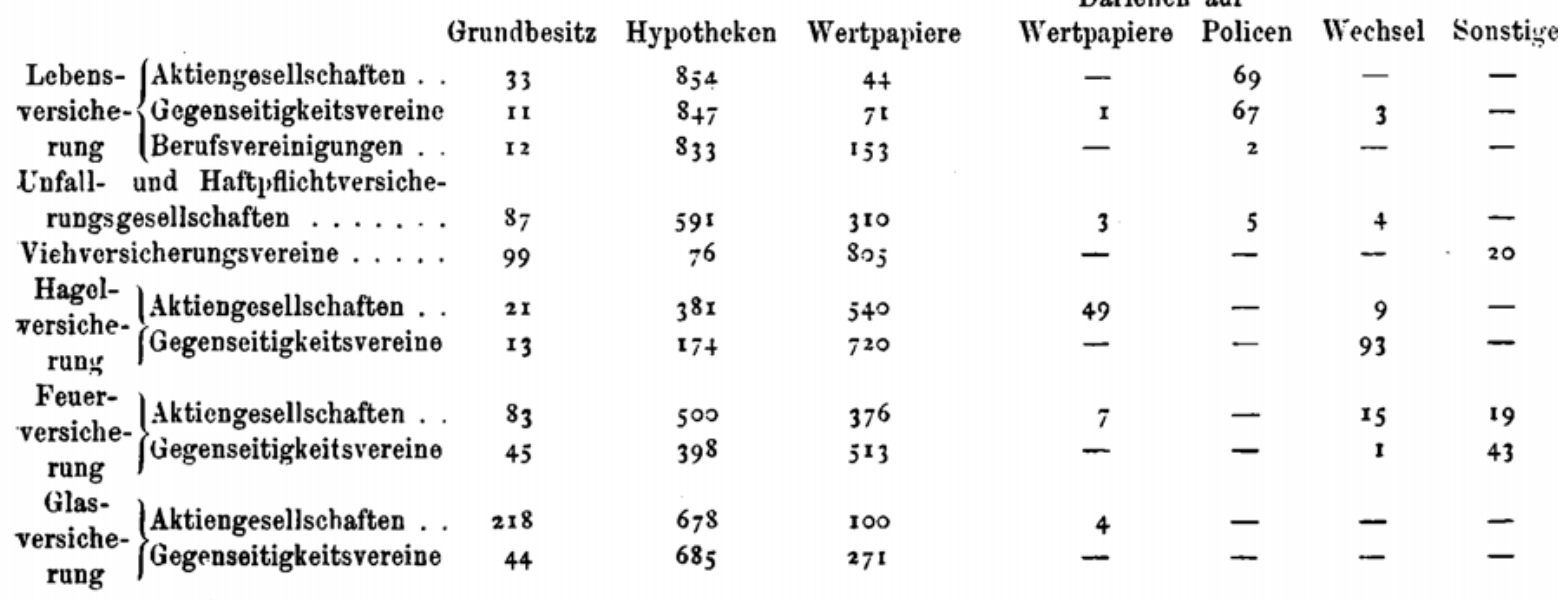

Die deutschen Lebensversicherungsunternehmungen bevorzugen in allererster Linie Hypotheken, in denen sie durchschnittlich 80 bis 90 Prozent ihrer Kapitalien angelegt haben. Die Unternehmungen der anderen Zweige können viel leichter in die Lage kommen, einen im Verhältnisse zum ganzen Besitze beträchtlichen Teil der Anlagen flüssig machen zu müssen. Daher treten bei ihnen die Wertpapiere in den Vordergrund, und zwar umsomehr, je kleiner die Reserven für unvorhergesehene Fälle im Verhältnisse zu dem Risiko sind; das tritt besonders deutlich bei der Hagelversicherung hervor.

Ganz allgemein werden Anlagen in Wertpapieren von den deutschen Gegenseitigkeitsvereinen mehr gesucht als von den Aktiengesellschaften. Die für alle Kreise der Bevölkerung bestimmten Gegenseitigkeitsvereine in der Lebensversicherung unterscheiden sich von den Lebensversicherungsaktiengesellschaften allerdings nur wenig; die ebenfalls auf dem Grundsatze der Gegenseitigkeit beruhenden Versicherungseinrichtungen von Berufsvereinigungen haben schon verhältnismäßig mehr Wertpapiere in ihrem Besitz, und wenn hierfür zur Zeit auch keine Zahlen angegeben werden können, so kann doch das Kaiserliche Aufsichtsamt aus seiner Erfahrung sagen, daß das in noch viel höherem Maße bei den meisten kleinen Pensions- und Sterbekassen der Fall ist, die in dieser Statistik nur nebenher erwähnt werden. Diese Versicherungseinrichtungen werden vielfach von Personen geleitet, die in Geldgeschäften wenig bewandert sind, es ist daher nur zu begrüßen, wenn sie sich mit dem schwierigeren und gefahrvolleren, wenn auch mehr Ertrag versprechenden Hypothekengeschäfte nicht befassen.

Bei den Gegenseitigkeitsvereinen der anderen Versicherungszweige mag es gelegentlich auch vorkommen, daß sie aus demselben Grunde vorzugsweise Wertpapiere ankaufen, in der Regel aber wird die Ursache darin liegen, daß sie ihre Mittel leichter müssen flüssig machen können als die Aktiengesellschaften, weil ihre Deckungsmittel verhältnismäßig kleiner zu sein pflegen und auch sein dürfen, da die Vereine ihre Hauptsicherheit in der gegenseitigen Haftung ihrer Mitglieder finden.

Die Kapitalanlagen der deutschen Versicherungsunternehmungen haben im Laufe des Berichtsjahrs um netto 202,69 Millionen Mark zugenommen, und zwar ist gewachsen der Grundbesitz um 2,84 Millionen, der Hypothekenbesitz um 226,52 Millionen, die Wertpapiere um 10,74 Millionen und die Policedarlehen um 14,15 Millionen, dagegen haben abgenommen die Darlehen auf Wertpapiere um 0,76 Millionen, der Besitz an Wechseln um 0,49 Millionen und die sonstigen Anlagen um 0,31 Millionen. Sieht man von den Darlehen auf Wertpapiere und von den Policedarlehen ab, die den öffentlichen Geldverkehr nicht direkt 
berühren, so haben die deutschen Versicherungsunternehmungen, soweit sie der Reichsaufsicht unterstehen, dem Geldmarkt im Jahre 1903 nach Absetzung aller Veräußerungen 239,30 Millionen Mark zugeführt.

Die

Verteilung

des

Gewinns

In der Bilanz sind bei jedem Versicherungszweige die Verluste, die einige Unternehmungen zu verzeichnen hatten, gegen die Gewinne der anderen Gesellschaften aufgerechnet, so ergiebt sich für die Gesamtheit der deutschen Versicherungsunternehmungen ein Netto-Gewinn von 131,180 Millionen Mark. Fragt man aber nach der Verteilung des Gewinns, und diese Frage muß hier aufgeworfen werden, schon um zu erfahren, in wie hohem Maße die Versicherten an dem Gewinne teilnehmen, so kommt der Gewinn aller derjenigen Gesellschaften in Betracht, die mit finanziellem Erfolge gearbeitet haben, das sind 136,246 Millionen Mark. Wie dieser Betrag verteilt worden ist, zeigt folgende Übersicht.

\begin{tabular}{|c|c|c|c|c|c|c|c|c|}
\hline $\begin{array}{l}\text { Von dem Gewinne sind in } 1000 \AA \\
\text { abgeführt an }\end{array}$ & $\begin{array}{c}\text { den } \\
\text { Kapital- } \\
\text { reserve- } \\
\text { fonds }\end{array}$ & $\begin{array}{l}\text { sonstige } \\
\text { Rèserven }\end{array}$ & $\begin{array}{l}\text { den } \\
\text { Be- } \\
\text { amten- } \\
\text { fonds }\end{array}$ & $\begin{array}{c}\text { die } \\
\text { Aktionäre } \\
\text { oder } \\
\text { Garanten }\end{array}$ & $\begin{array}{l}\text { Tan- } \\
\text { tiemen }\end{array}$ & $\begin{array}{c}\text { die } \\
\text { Ver- } \\
\text { sicherten }\end{array}$ & $\begin{array}{l}\text { ander- } \\
\text { weit }\end{array}$ & $\begin{array}{l}\text { Vortrag } \\
\text { auf nene } \\
\text { Rechnang }\end{array}$ \\
\hline Lebensversicherung . . . . . . & I 472 & $5 \times 54$ & 430 & 4688 & 1992 & 74688 & I 815 & 234 \\
\hline Unfall- und Haftpflichtversicherung . & 99 & 679 & 150 & I 944 & 560 & 1880 & 113 & 401 \\
\hline Viehversicherung $\ldots \ldots \ldots \ldots$ & 63 & 12 & $\mathbf{I}$ & - & - & - & - & 3 \\
\hline Hagelversicherung . . . . . . & 277 & 659 & 80 & 167 & 189 & - & - & 86 \\
\hline Fenerversicherung usw. . . . . . . & 1270 & 3639 & 890 & $1 \times 136$ & I 502 & 16595 & 836 & 858 \\
\hline Glasversicherung $\ldots \ldots \ldots$ & 40 & 82 & 23 & 301 & 120 & 26 & 61 & $3 \mathbf{x}$ \\
\hline Zusammen . . . & 3221 & 10225 & 1574 & 10236 & 4303 & 03189 & 2825 & 1613 \\
\hline
\end{tabular}

Das ausländische Geschät

der deutschen und das deutsche Geschäft landische Gesell-

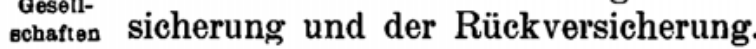

Schließlich mag hier noch eine Gegenüberstellung der Prämien und S̈chäden, beide einschließlich der Anteile der Rückversicherer, bei den verschiedenen Versicherungszweigen Platz finden, die einerseits das ausländische Geschäft der deutschen Gesellschaften und andererseits das deutsche Geschäft der ausländischen Gesellschaften betreffen. Es sei aber besonders hervorgehoben, $\mathrm{da} \beta$ aus den Endzahlen keineswegs Schlüsse auf die Verhältnisse des Versicherungsgeschäfts überhaupt gezogen werden dürfen; dazu fehlen die Zahlen für die ganz vornehmlich internationalen Betriebe der Transportver-

\begin{tabular}{|c|c|c|c|c|}
\hline & \multicolumn{2}{|c|}{$\begin{array}{l}\text { Ausländisches Geschäft der } \\
\text { deutschen Gesellschaften }\end{array}$} & \multicolumn{2}{|c|}{$\begin{array}{l}\text { Deutsches Geschäft der aus } \\
\text { ländischen Gesollschaften }\end{array}$} \\
\hline & $\begin{array}{l}\text { Prämien } \\
1000 \mathscr{A}\end{array}$ & $\begin{array}{l}\text { Schäden } \\
1000 \mathscr{A l}\end{array}$ & $\begin{array}{l}\text { Prämien } \\
1000 \mathscr{M}\end{array}$ & $\begin{array}{l}\text { Schäden } \\
1000 \varkappa \mathscr{K}\end{array}$ \\
\hline Lebensversicherung $\ldots \ldots \ldots \ldots$ & 25379 & 10287 & 32699 & 17028 \\
\hline Unfall- und Haftpflichtversicherung. . . & 6532 & 3982 & 10961 & 5339 \\
\hline Viehversicherung. . . . . . . . & 117 & 87 & - & - \\
\hline Hagelversicherung $\ldots \ldots \ldots \ldots$ & 1208 & 641 & - & - \\
\hline Feuerversicherung $\ldots \ldots \ldots \ldots$ & 60403 & 36532 & 20879 & 10722 \\
\hline Einbruchdiebstahlversicherung . . . . . & 627 & 163 & 322 & 48 \\
\hline Glasversicherung . . . . . . . . . & 394 & 217 & 159 & 149 \\
\hline Sonstige Versicherungen $\ldots \ldots \ldots$ & 66 & 62 & - & - \\
\hline Zusammen ... & .94726 & $5 \times 97 \times$ & 65020 & 33286 \\
\hline
\end{tabular}




\section{Gruppe I, Lebens- und Krankenversicherung.}

Im Jahre 1903 standen auf dem Gebiete der Lebens- und der Krankenversicherung Die Unter: 28 deutsche Aktiengesellschaften und eine große Zahl deutscher Gegenseitigkeitsvereine $\underset{\text { gen }}{\text { nehmur }}$ unter Reichsaufsicht.

Während die 28 Aktiengesellschaften und 22 große Gegenseitigkeitsvereine ihren Geschäftsbetrieb für alle Kreise der Bevölkerung offen halten, versichert die Mehrzahl der Gegenseitigkeitsvereine nur die Angehörigen mehr oder weniger eng begrenzter Kreise, weshalb sie meist von verhältnismäßig geringer Bedeutung sind. Nur einer von diesen (der Preußische Beamtenverein in Hannover) hat einen so großen Versicherungsbestand gewonnen, daß man ihn den anderen Gegenseitigkeitsvereinen, die eine Beschränkung auf bestimmte Kreise nicht kennen, an die Seite stellen kann; deshalb ist er diesen auch hier wie in früheren Statistiken zugesellt worden. Von den anderen Gegenseitigkeitsvereinen für beschränkte Personengruppen sind in die Tabellen dieser Statistik nur einige der größeren Versicherungseinrichtungen von Berufsvereinigungen aufgenommen. Die große Mehrzahl von Pensions- und Sterbekassen, die vielfach nur die Versicherung der Angestellten bestimmter gewerblicher Firmen bezwecken, sind wie im vorigen Jahre aus den Tabellen ganz fortgelassen; sie werden auch weiter unten im Texte nur ganz summarisch behandelt werden.

Auch die größeren Pensions- und Sterbekassen gehören ihrem ganzen Betriebe nach eher zu der kleinen Versicherung, als zu der großen (regulären), sie sind hier aber für sich behandelt und in Abweichung von der vorjährigen Statistik als Versicherungseinrichtungen von Berufsvereinigungen zusammengefaßt. Die Bezeichnung „kleine Versicherung“ ist im Folgenden lediglich für die kleine Versicherung der im allgemeinen allen Bevölkerungsklassen zugänglichen Unternehmungen verwendet.

Von allen dem Aufsichtsant unterstehenden deutschen Lebensversicherungsvereinen auf Gegenseitigkeit sind in den Tabellen dieser Statistik 22 allgemeinen Charakters, oder, wenn man will, größere Gegenseitigkeitsvereine, und 16 Versicherungsvereine von Berufsvereinigungen behandelt.

Eine Aktiengesellschaft - Nordstern, Unfall- und Alters-Versicherungs-Aktien -Gesellschaft in Berlin - und ein Gegenseitigkeitsverein - Allgemeine Deutsche Versicherungs-Gesellschaft Schutz und Trutz (Sterbekasse) in Dresden - haben sich, soweit Versicherungen der Gruppe I in Frage kommen, nur an der kleinen Versicherung beteiligt.

Gegenüber der vorjährigen Statistik sind in der diesmaligen hinsichtlich der behandelten Unternehmungen folgende Änderungen eingetreten. Die Hannovera ist auf die Arminia übergegangen, ebenso ist die Dresdener Allgemeine Versicherungsanstalt mit der Augusta und die Leipziger Gegenseitigkeit, Versicherungs-Gesellschaft von 1855, mit der Teutonia fusioniert. Neu hinzugekommen ist bei den allgemeinen Unternehmungen die Alemannia, Versicherungs-Gesellschaft auf Gegenseitigkeit zu Leipzig, die bei der vorjährigen Statistik fortgelassen werden mußte, weil die für das Jahr 1902 gelieferten Geschäftsausweise unzulänglich waren. Bei den Versicherungseinrichtungen von Berufsvereinigungen sind neu aufgenommen die Altersversorgungs- und Invaliditätskasse des Verbandes deutscher Handlungsgehülfen zu Leipzig, die Witwen- und Waisenkasse desselben Verbandes, die Pensionskasse des Deutschen Privat-Beamten-Vereins in Magdeburg und die Witwenkasse desselben Vereins.

Von den ausländischen Lebensversicherungsgesellschaften haben im Jahre $190323 \mathrm{der}$ Reichsaufsicht unterstanden. Die vorliegende Statistik berichtet indessen nur über 22; zu den in der vorjährigen Arbeit behandelten ist The Mutual Life Insurance Company in 
New-York hinzugekommen, sie ist im Jahre 1842 gegründet worden und hat den Geschäftsbetrieb in Deutschland im Jahre 1886 aufgenommen, ihn aber im Jahre 1904 eingestellt. Die Equitable in New-York hat aus demselben Grunde wie im Vorjahr, auch in diesem Jahre noch fortgelassen werden müssen.

Von den 28 deutschen Aktiengesellschaften betrieben im Jahre r903 neben der Lebensversicherung 13 auch die Unfallversicherung, 9 die Haftpflichtversicherung, je 2 die Feuerversicherung, die Kautionsversicherung (in ganz bescheidenem Umfange), die Transportversicherung und 1 die Versicherung gegen Einbruchdiebstahl. Von den Gegenseitigkeitsvereinen hat nur einer (der Allgemeine Deutsche Versicherungs-Verein in Stuttgart) außer in der Lebensversicherung noch in der Unfall- und Haftpflichtversicherung und in einigen kleinen Nebenzweigen Geschäfte gemacht.

Von den für alle Kreise der Bevölkerung zugänglichen deutschen Unternehmungen unter Reichsaufsicht sind im Jahre 1903 tätig gewesen

\section{in der großen Versicherung}

in der Versicherung anf den Todesfall 27 Aktiengesellschaften und 20 Gegenseitigkeitsvereine zus. 47 Unternehmungen

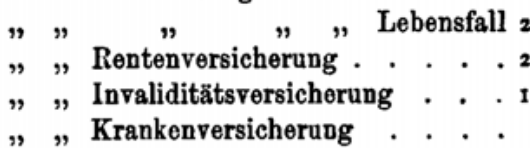

" $\quad$ "1

$\begin{array}{lrr}\Rightarrow & 44 & \\ \Rightarrow & 36 & \\ " & 22 & \end{array}$

in der kleinen Versicherung

in der Volksversicherung . . . $9^{\prime}$ Aktiengesellschaften und 3 Gegenseitigkeitsvereine zusammen 2 Unternehnungen in der sonstigen kleinen Versich.

Die Nürnberger Lebensversicherungs-Bank hat die kleine Versicherung erst in dem Berichtsjahr aufgenommen.

Von den 22 ausländischen Gesellschaften, über welche diese Statistik berichtet, haben im Deutschen Reiche gearbeitet 21 in der Versicherung auf den Todesfall, 21 in der Versicherung auf den Lebensfall, 18 in der Rentenversicherung, 2 in der Invaliditätsversicherung und 5 in der Volksversicherung; von diesen hat jedoch eine im Laufe des Berichtsjahrs ihr kleines deutsehes Volksversicherungsgeschäft eingestellt.

Trennung der groBen von der kleinen Veraicherung,

Volksversicherung
Vom Standpunkte der Statistik ist eine möglichst vollständige Trennung des großen (regulären) Geschäfts von dem kleinen Gẻschäfte wünschenswert; denn der Betrieb ist verschieden, und die Versicherungen dienen zum Teil verschiedenen Zwecken.

Nun haben nach den Rechnungsvorschriften diejenigen Versicherungsunternehmungen, deren Geschäftsbetrieb die sogenannte kleine Lebensversicherung (Volksversicherung, Arbeiter-, Sterbekassen- usw. Versicherung) ohne ärztliche Untersuchung oder mit unvollständiger ärztlicher Untersuchung in der Art umfaßt, daß für diesen Versicherungsbetrieb besondere geschäftliche Einrichtungen und besondere von den sonstigen Geschäftsgrundsätzen der Unternehmung wesentlich abweichende allgemeine Versicherungsbedingungen, Tarife, Provisionssätze eingeführt sind, für diesen Versicherungsbetrieb besondere Nachweisungen namentlich auch über den Versicherungsbestand und seine Bewegung sowie hinsichtlich der Gewinn- und Verlustrechnung aufzustellen. Sofern aber die Unternehmungen für diesen Versicherungsbetrieb keine derartige besondere Einrichtungen haben, sind sie berechtigt, die kleine Versicherung mit der großen zusammen nachzuweisen.

Unter diesen Umständen ist es nicht möglich gewesen, die kleine Versicherung vollständig aus dem gesamten Geschäfte herauszuschälen. Mehrfach konnte das nur bei dem Versicherungsbestande, nicht aber auch bei der Bewegung des Bestandes während des Berichtsjahrs geschehen. Wo die Scheidung mit Hilfe der dem Aufsichtsamt eingereichten Übersichten möglich war, ist sie vorgenommen worden, obwohl dadurch eine gewisse Unebenheit zwischen den einzelnen Abschnitten dieser Statistik entstehen mußte; es erschien aber besser, das mit in den Kauf zu nehmen, als auf eine strenge Scheidung 
der beiden Betriebsarten vollständig zu verzichten. Da, wo die Unternehmungen für die kleine Versicherung besondere geschäftliche Einrichtungen obengenannter Art geschaffen haben, und wo mithin das kleine Geschäft von dem großen vollständig getrennt gehalten werden kann, wird in dieser Statistik die kleine Versicherung als "Volksversicherung" bezeichnet, wo das nicht der Fall, als "sonstige kleine Versicherung“.

Der Beobachtungsstoff ist wie in vorigen Jahre folgendermaken angeordnet:

Es ist zunächst der Versicherungsbestand am Ende des Jahres 1903 behandelt ${ }^{\text {des Stoffes }}$ (Tabellen I 1 bis I 20), danach folgt die Bewegung des Versicherungsbestandes im Berichtsjahre (Tabellen 121 bis I 32), dann die Gewinn- und Verlustrechnung (Tabellen I 33 bis I 41) und endlich die Bilanz für Fnde 1903 (Tabellen I 42 bis I 54).

In den einzelnen Abschnitten werden nacheinander vorgeführt: die großen deutschen Unternehmungen (Aktiengesellschaften und Gegenseitigkeitsvereine) zuerst mit ihrer grolen, dann mit ihrer kleinen Versicherung, danach die Versicherungseinrichtungen einiger größerer deutscher Berufsvereinigungen (Pensionskassen und Sterbekassen) und endlich die ausländischen Unternehmungen. Dabei wird von den deutschen Unternehmungen in den ersten drei Abschnitten immer das Lebensversicherungsgeschäft (inländisches und ausländisches) für sich betrachtet, während die Bilanz nur für das Gesamtgeschäft (Lebensversicherung nebst allen Nebenzweigen) gegeben wird. Ton den ausländischen Unternehmungen wird der Bestand und die Bewegung nur für das deutsche Geschäft zusammengestellt, die Betriebsrechnung dagegen bezieht sich auf das ganze Lebensversicherungsgeschäft und die Bilanz auf das Gesamtgeschäft.

Hieran schließt sich eine Vergleichung des ausländischen Geschäfts der deutschen Unternehmungen mit dem deutschen Geschäfte der ausländischen Gesellschaften in den wichtigsten Punkten und endlich folgen einige Angaben über die dem Aufsichtsamt unterstehenden kleinen Pensions- und Sterbekassen.

\section{Der Versicherungsbestand am Ende des Jahres 1903.}

Über den gesamten Bestand der unter Reichsaufsicht stehenden deutschen Lebens- Deutsche versicherungsunternehmungen, die dem großen Publikum dienen, geben die Tabellen I 1 allgemeine bis I 11 ausführlichen Aufschluß; hier sollen daraus nur die hauptsächlichsten für die as das unGesamtheit der Anstalten geltenden Ergebnisse zusammengestellt werden.

Am Ende des Berichtsjahrs waren an überhaupt übernommenen Versicherungen mittelbare in Kraft:

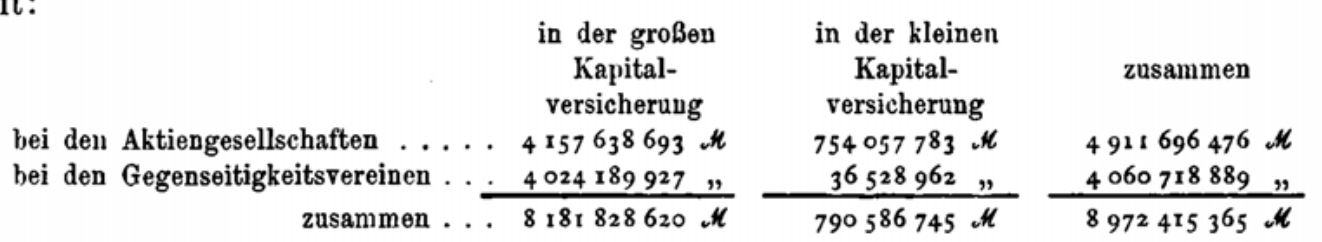

Hierin sind alle diejenigen Beträge doppelt gerechnet, welche von einer Gesellschaft, die in unserer Statistik behandelt wird, versichert und bei einer ebensolchen Gesellschaft in Rückdeckung gegeben sind; diese Beträge selbst zu bestimmen ist nicht angängig, wohl aber lassen sich alle in Rückdeckung gegebenen Summen bestimmen; setzt man diese ab, so erhält man diejenigen Versicherungssummen, welche die Gesellschaften unserer Statistik auf eigenes Risiko behalten haben:

\begin{tabular}{|c|c|c|c|}
\hline & $\begin{array}{l}\text { große Kapital- } \\
\text { rersicherung }\end{array}$ & $\begin{array}{l}\text { kleine Kapital- } \\
\text { versicherung }\end{array}$ & zusammen \\
\hline $\begin{array}{l}\text { Aktiengesellschaften } \ldots \ldots \ldots \ldots \ldots \\
\text { Gegenseitigkeitsvereine } \ldots \ldots \ldots \ldots\end{array}$ & $\begin{array}{l}3969071870 \mathrm{NK} \\
4002524394 \pi \\
\end{array}$ & $\begin{array}{r}738525539 \boldsymbol{M} \\
36528962 \mu \\
\end{array}$ & $\begin{array}{l}4707597409 \\
4039053356\end{array}$ \\
\hline $\begin{array}{r}\text { zusammen } \ldots \ldots \\
\text { Versicherungs-Statistik } 1903 .\end{array}$ & 7971596264 ok & $775054501 \mu$ & 8746650765 \\
\hline
\end{tabular}


Mehr Interesse gewinnt die Behandlung des Verhältnisses der Versicherungen für eigene Rechnung zu den überhaupt übernommenen Versicherungen, wenn man die Kapitalversicherung in Todesfallversicherung und in Versicherung auf den Lebensfall trennt; dabei soll die Rentenversicherung (ohne Invaliditätsrenten) gleich mit in Betracht gezogen werden. Nach. Tabelle I 2 waren in der großen Versicherung folgende Beträge überhaupt in Versicherung genommen:

$$
\begin{aligned}
& \text { in der Kapitalversicherung auf in der Renten- } \\
& \text { den T'odesfall den Lebensfall versicherung } \\
& \text { von den Aktiengesellschaften... } 37293^{83} 621 \text { oth } \quad 428253181 \text { oh } \quad 1333^{8} 309 \text { oth }
\end{aligned}
$$

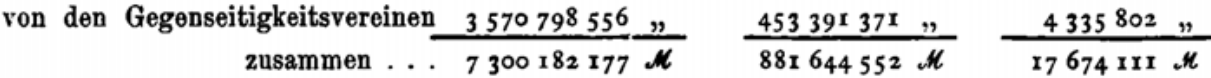

Für eigene Rechnung waren nach Tabelle I 3 davon behalten:

von den Aktiengesellsch. . $3547422453 \boldsymbol{N}$ oder $95,2 \% \quad 421649417 \boldsymbol{k}$ oder $98,5 \% \quad 12991480 \boldsymbol{N}$ oder $97,4 \%$

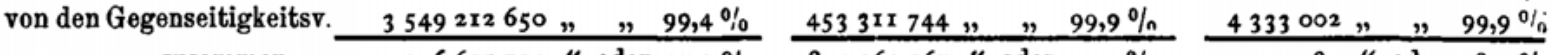
zusammen . . . $7096635103 \mu$ oder $97,2 \% \frac{}{87496116 \times \mu \text { oder } 99,2 \%} 17324482 \mu$ oder $98,0 \%$

In der kleinen Versicherung haben nur einige Aktiengesellschaften bei einem Teile ihrer Volksversicherungen von der Rückversicherung Gebrauch gemacht. Von $724555272 \mathscr{M}$ Versicherungssumme auf den Todesfall sind 709334979 CH. oder 97,9\% und von 1632085 c Versicherungssumme auf den Lebensfall sind $1320134 \mathscr{N}$. oder 80,9\% für eigene Rechnung behalten worden.

Die Rückversicherung spielt hiernach in der Lebensversicherung im ganzen nur eine bescheidene Rolle, zumal bei den Gegenseitigkeitsvereinen. Am meisten findet sie in dem großen Geschäfte bei der Kapitalversicherung auf den Todesfall Anwendung, am wenigsten bei der Lebensfallversicherung, ungefähr in der Mitte steht die Rentenversicherung. Daß sie bei der Lebensfall- und der Rentenversicherung selten in Anspruch genommen wird, liegt in der Natur dieser Versicherungsarten, und daß sie bei der kleinen Versicherung nicht häufiger vorkommt, in der Kleinheit der einzelnen Risiken. Man würde sie hier noch seltener finden, wenn nicht einige Gesellschaften vertragsmäßig verpflichtet wären, von jeder von ihnen abgeschlossenen Versicherung einen bestimmten Prozentsatz in Rückdeckung zu geben (sog. Quotenrückversicherung).

Fragt man dann weiter, wie sich der Versicherungsbestand der deutschen Gesellschaften aus selbst abgeschlossenen und aus in Rückdeckung übernommenen Versicherungen zusammensetzt, so ersieht man aus den Tabellen I 4 bis I 8 und I 10 und I 11, daß die Gegenseitigkeitsvereine Ende 1903 nur vereinzelt einige Versicherungen auf den "Todesfall und geringfügige Jahresrenten in Rückdeckung genommen, und daß auch die Aktiengesellschaften im großen und ganzen nur wenig mittelbare Geschäfte abgeschlossen haben.

Eine Zusammenstellung der für die Rückversicherung überhaupt in Betracht kommenden Beträge zeigt für die deutschen Unternehmungen folgendes Bild:

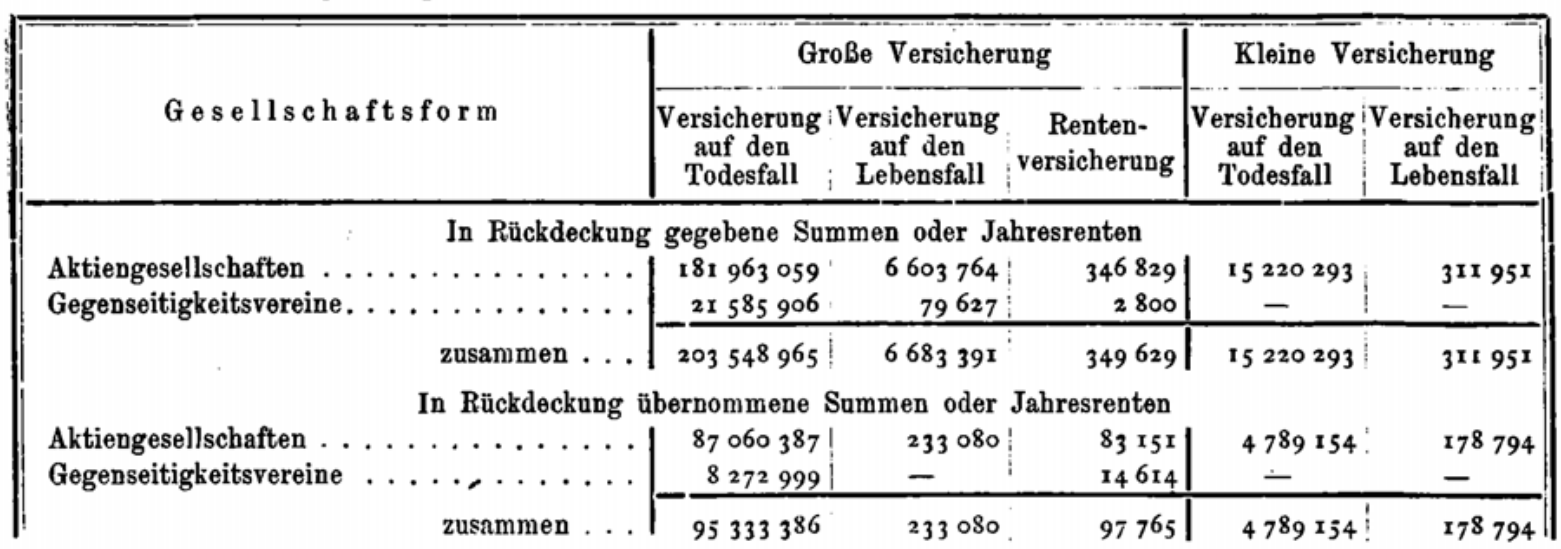


In der großen Todesfallversicherung und in der kleinen Versicherung ist hiernach von den unter Reichsaufsicht stehenden Unternehmungen doppelt bis dreimal so viel in Rückdeckung gegeben wie von denselben Gesellschaften in Rückdeckung übernommen ist, bei den anderen großen Versicherungen treten die in Rückdeckung übernommenen Beträge noch mehr zurück.

In unseren Tabellen sind auch die mit Gewinnbeteiligung abgeschlossenen Ver-b) die versicherungen von denen ohne Gewinnbeteiligung vollständig getrennt, um zunächst zu gicherunzeigen, wie sich das Verhältnis der einen zu den anderen bei den einzelnen Versicherungs- und ohne arten gestaltet, und um mit Hilfe der späteren Statistiken die weitere Entwickelung dieses cewinnVerhältnisses verfolgen zu können. Daß Versicherungen mit Gewinnbeteiligung bei den Aktiengesellschaften verhältnismäß̣ig weniger vertreten sind als bei den Gegenseitigkeitsvereinen liegt in der Natur der Gesellschaften. Trotzdem scheint es nicht überflüssig, wenigstens für die selbst abgeschlossenen Versicherungen die V'erhältniszahlen hier zusammenzustellen.

\begin{tabular}{|c|c|c|c|c|c|c|c|c|}
\hline \multirow{3}{*}{$\begin{array}{l}\text { Ani Gewinne waren beteiligt bei den } \\
\text { selbst abgeschlossenen Verträgen }\end{array}$} & \multicolumn{4}{|c|}{ Aktiengesellschaften } & \multicolumn{4}{|c|}{ Gegenseitigkeitsvereine } \\
\hline & \multicolumn{2}{|c|}{$\begin{array}{c}\text { ron } \\
1000)^{\text {Policen }}\end{array}$} & \multicolumn{2}{|c|}{$\begin{array}{l}\text { von } \\
1000 \text { d }\end{array}$} & \multicolumn{2}{|c|}{$\begin{array}{c}\text { von } \\
1000 \text { Policen }\end{array}$} & \multicolumn{2}{|c|}{$\begin{array}{c}\text { von } \\
1000 \mathrm{~A}\end{array}$} \\
\hline & 1902 & 1903 & 1902 & I903 & 1902 & 1903 & 1902 & 1903 \\
\hline der Todesfallversicherung & 815 & 818 & 897 & 900 & 993 & 993 & 998 & 998 \\
\hline der Versicherung auf den Lebensfall . & 325 & 333 & 286 & 282 & 923 & 920 & 888 & 885 \\
\hline der Rentenversicherung . . . . . . & $\circ$ & $\circ$ & $\circ$ & $\circ$ & 863 & 852 & 730 & 720 \\
\hline 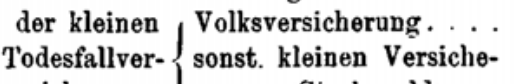 & 650 & 661 & 715 & 732 & 976 & 957 & 995 & 987 \\
\hline sicherung $\mid$ rung (Sterbegeld)... & 192 & 205 & 256 & $27^{2}$ & $29 t$ & 293 & 191 & 191. \\
\hline
\end{tabular}

Die Unterschiede zwischen beiden Jahren sind nicht beträchtlich. Bei den Aktiengesellschaften ist die Gewinnbeteiligung bei den Versicherungen auf den Lebensfall etwas zurückgegangen, sonst hat sie überall zugenommen. Bei den Gegenseitigkeitsvereinen ist sie bei der Versicherung auf den Todesfall und bei der Sterbegeldversicherung so gut wie ungeändert geblieben, bei den übrigen Versicherungsarten hat sie abgenommen. Im übrigen gilt für Ende 1903 dasselbe wie für Ende r9o2. Die Versicherungen mit Gewinnbeteiligung sind, wenn man von der kleinen Versicherung zunächst absieht, am häufigsten bei der Versicherung auf den Todesfall, und hier nähern sich die Verhältniszahlen der Aktiengesellschaften denen der Gegenseitigkeitsvereine in bemerkenswerter Weise. Bei den Versicherungen auf den Lebensfall findet sich die Gewinnbeteiligung schon seltener, wenn auch der Unterschied bei den Gegenseitigkeitsvereinen sehr viel kleiner ist, als bei den Aktiengesellschaften. Beachtenswert ist vielleicht, daß bei beiden Arten von Unternehmungen in der Todesfallversicherung die Policen der Zahl nach mit einem etwas geringeren Prozentsatz am Gewinn Anteil haben als die Versicherungssummen, während bei der Versicherung auf den Lebensfall das Entgegengesetzte der Fall ist. Die Versicherungen mit Anteil am Gewinne liegen also hinsichtlich der Höhe der Versicherung beim Todesfall über, beim Lebensfall unter dem Durchschnitte. Rentenversicherungen werden von den Aktiengesellschaften nur ausnahmsweise mit Gevinnbeteiligung der Versicherten abgeschlossen; von den Gegenseitigkeitsvereinen geschieht das naturgemäß sehr viel mehr; doch finden sich - soweit unsere Zahlen, bei denen Angaoen über die zahlreichen Versicherungen steigender Renten fehlen, ein Urteil zulassen - bei dieser Versicherungsart verhältnismäßig immer noch viel Versicherungen ohne Gewinnanteil. Bei der kleinen Versicherung fällt besonders der geringe Prozentsatz der an Gewinne beteiligten sonstigen kleinen Versicherung (Sterbegeldversicherung) bei den Gegenseitigkeitsvereinen auf; sie unterscheiden sich in dieser Beziehung kaum merklich von den Aktiengesellschaften. 
$\mathrm{XX}$

Gruppe I

c) Durch- Der durchschnittliche Betrag einer Versicherung ist nur für selbst abgeschlossene sobnitts-
werte Versicherungen berechnet, da es hier nur darauf ankommt, zu erfahren, auf welche Summen die Versicherungen durchschnittlich lauten, die von den Versicherten mit dem Erstversicherer abgeschlossen werden.

Durchschnittliche Versicherungssuṃme für eine selbst abgeschlossene

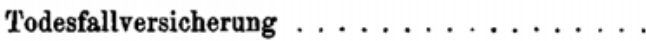

Lebensfallversicherung . . . . . . . . . . .

Rentenversicherung (Jahresrente) . . . . . . . . .

kleine Todes- (Volksversicherung . . . . . . . .

fallversicherung sonstige kleine Vers. (Sterbegeld)

kleine Lebens- Volksversicherung . . . . . . . . .

fallversicherung sonstige kleine Versicherung . .

\begin{tabular}{|c|c|c|c|}
\hline Akti & eng & lschaft & \\
\hline 190 & & 1903 & \\
\hline $40+9$ & $\mathscr{M}$ & 4031 & th \\
\hline 726 & $"$ & 1722 & " \\
\hline 480 & " & $47^{8}$ & " \\
\hline $1 ; 6$ & " & 175 & $\eta$ \\
\hline $43 \mathrm{I}$ & $"$ & 437 & $"$ \\
\hline 270 & $"$ & 260 & $\eta$ \\
\hline 491 & $"$ & $49 \mathrm{I}$ & " \\
\hline
\end{tabular}

\begin{tabular}{|c|c|c|c|}
\hline \multicolumn{4}{|c|}{ Gegenseitigkeitsvereine } \\
\hline 190 & & 1903 & \\
\hline 5 ror & $M$ & 5188 & H \\
\hline I 446 & $\eta$ & I $46 \mathrm{I}$ & \\
\hline 183 & $"$ & 193 & , \\
\hline 181 & $\eta$ & 175 & \\
\hline 315 & $"$ & 318 & \\
\hline- & $"$ & - & \\
\hline- & $"$ & - & \\
\hline
\end{tabular}

Im Vergleiche mit dem Vorjahr ist danach im Berichtsjahre die durchschnittlich auf eine Police entfallende Summe bei den Aktiengesellschaften mit Ausnahme der Sterbegeldversicherung etwas herabgegangen; bei den Gegenseitigkeitsvereinen hat sie dagegen mit Ausnahme der Volksversicherung in etwas höherem Maße zugenommen.

d) In-

validitats-

Die Versicherung auf den Invaliditätsfall wird nur von wenigen Gesellschaften als

ver- selbständige Versicherung betrieben, zumeist tritt sie als Zusatzversicherung zu der ge-

sicherung mischten Kapitalversicherung auf, indem für den Fall der Invalidität Prämienfreiheit oder Prämienfreiheit in Verbindung mit einer Rente (meist 5 oder 10 Prozent des versicherten Kapitals) bis zum Ablaufe der Versicherung ausbedungen wird. Diese Art des Betriebs hat es mit sich gebracht, daß über den Unfang der Invaliditätsversicherung der großen Gesellschaften auch jetzt noch nur sehr mangelhafte Angaben gemacht werden können. Einige Unternehmungen führten bis dahin die mit Invaliditätsversicherung verbundenen Kapitalversicherungen in ihren Büchern nicht getrennt von den reinen Kapitalversicherungen, und deshalb konnten sie den Stand und die Bewegung der Invaliditätsversicherung überhaupt nicht oder doch nur unvollständig nachweisen. Besonders unzuverlässig sind die Angaben über den Jahresbetrag der für den Invaliditätsfall versicherten Renten, da dieser nicht überall in derselben Weise festgestellt worden ist. Als Jahresbetrag der versicherten Invaliditätsrente sollte bei Kapitalversicherungen, die im Invaliditätsfalle nur Prämienfreiheit gewähren, die Bruttoprämie der Versicherung gelten und bei Kapitalversicherungen, die im Invaliditätsfall außer der Befreiung von der Prämienzahlung noch eine Rente gewähren, diese Rente vermehrt um die Bruttoprämie. Trotz der mancherlei Bedenken, die hiernach gegen die Zahlen in unseren Tabellen über die Invaliditätsversicherung erhoben werden können, und die erst allmählich werden fortgeräumt werden können, schien es doch zweckmäßig, sie mit zu veröffentlichen.

•)Krankon- Die Krankenversicherung eignet sich weniger als jede andere Versicherungsart zum ver- Betriebe von einer Zentrale aus, sie erfordert, wenn sie ohne übergroße Gefährdung des Versicherers durchgeführt werden soll, eine weitgehende Kontrolle der Versicherten, die ohne großen Verwaltungsapparat nicht $\mathrm{zu}$ ermöglichen ist. Tatsächlich befaßten sich im Jahre 1903 denn auch nur wenige Gesellschaften mit der Krankenversicherung, und ihr Bestand an Versicherungen war nur ein ganz geringer. Infolge der Fusion der Dresdener Allgemeinen Versicherungs-Anstalt mit der Augusta und der Leipziger Gegenseitigkeit mit der Teutonia erscheinen auf dem Gebiete der Krankenversicherung in der vorliegenden Statistik (Tabelle 18) vier Aktiengesellschaften und nur noch ein Gegenseitigkeitsverein. Bei diesen Unternehmungen waren Ende r9o3 insgesamt 9601 Versicherungen über $32549 \mathscr{M}$ tägliches Krankengeld und 623 sogenannte Gesundheitspflegeversicherungen in Kraft, bei denen im Krankheitsfalle kein Krankengeld, sondern nur freie Arznei und freie 
ärztliche Behandlung gewährt wird. Ein kleiner 'Teil der Krankenversicherungen ist mit Sterbegeldversicherungen verbunden; ein anderer Teil, insgesamt 506 Policen, erstreckt sich gleichzeitig auf den Invaliditätsfall.

Von den für begrenzte Kreise bestimmten Versicherungsunternehmungen sind, wie schon oben erwähnt, in die Tabellen dieser Statistik nur die einiger größerer Berufsvereinigungen aufgenommen. Obwohl sie in ihrer ganzen Einrichtung sehr erhebliche Unterschiede zeigen und sich deshalb nicht ohne Zwang gemeinsam behandeln lassen, sind zı den in der vorjährigen Statistik bearbeiteten zwölf Unternehmungen noch vier andere hinzugenommen worden. Dabei ist versucht worden, den Stoff übersichtlicher als im Vorjahr anzuordnen (Tabelle I 12), wenn dadurch die Vergleichung mit den früheren Mitteilungen auch etwas erschwert werden mag.

Von den fraglichen 16 Unternehmungen betrieben zehn die Versicherung auf den Todesfall (meist Sterbekassen), davon zwei auch die Versicherung auf den Lebensfall und drei die Rentenversicherung (meist Pensionsversicherung); die übrigen sechs befaßten sich lediglich mit der Pensionsversicherung.

Im ganzen waren bei diesen Versicherungseinrichtungen Ende r 903 in Kraft 192115 Policen über $100241944 \mathscr{H}$. auf den Todesfall (durchschnittlich 522 , /l für eine Versicherung), 123 Policen über $597700 \mathscr{M}$ auf den Lebensfall (durchschnittlich 4859 th für eine Versicherung) und 15503 Policen über 3764632 d Jahresrente (durchschnittlich 243 NI. Jahresrente für eine Versicherung). Außerdem liefen noch 2230 Versicherungen auf den Todesfall mit veränderlicher Höhe und 14530 Pensionsversicherungen mit steigender Rente.

Bei der einfachen Verwaltung der meisten solcher Versicherungsvereine ist es erklärlich, daß sie keine Versicherungen in Rückdeckung übernommen und auch nur geringfügige Beträge in Rückdeckung gegeben haben.

Von den ausländischen Versicherungsunternehmungen, die im Jahre 1903 im Deutschen Reiche Versicherungsgeschäfte betrieben haben, soll hier nur der Bestand ihres deutschen Geschäfts erörtert werden, wie er in den Tabellen I 13 bis I 20 erscheint. Dabei ist von einer Trennung zwischen Aktiengesellschaften und Gegenseitigkeitsvereinen abgesehen worden.

Was zunächst die Versicherungssummen betrifft, so waren am Ende des Berichtsjahrs

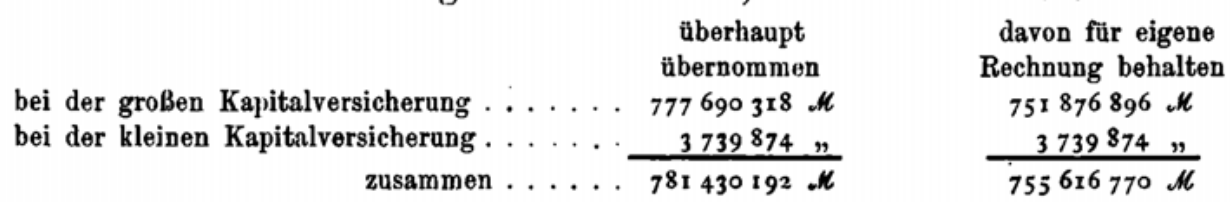

In welchen Maße hieran die Todesfall- und die Lebensfallversicherung beteiligt sind, ist aus folgender Übersicht zu entnehmen, in der zugleich auch die Rentenversicherung mit aufgeführt ist.

\begin{tabular}{|c|c|c|c|c|c|}
\hline Es waren & $\begin{array}{l}\text { überhaupt } \\
\text { übernommen }\end{array}$ & \multicolumn{4}{|c|}{$\begin{array}{l}\text { davon für eigene } \\
\text { Rechnung behalten }\end{array}$} \\
\hline Große $\quad$ in der Todesfallversicherung. & $654440633 \mu$ & 629499547 & $M$ & oder & $96,2 \%$ \\
\hline Versicherung $\left(\begin{array}{l}\text { in der Lebensfallversicherung } \ldots \ldots \ldots \\
\text { in der Rentenversicherung (Jahresrenten) } \ldots \ldots\end{array}\right.$ & 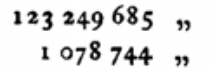 & $\begin{array}{r}122377349 \\
1066214\end{array}$ & $"$ & $"$ & $\begin{array}{l}99,3 " \\
98,9,\end{array}$ \\
\hline 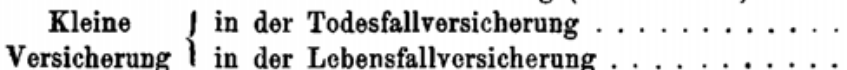 & $\begin{array}{r}355^{8} 548, \\
181326\end{array}$ & $\begin{array}{r}355^{8} 548 \\
\times 81326\end{array}$ & $"$ & $"$ & $100 "$ \\
\hline
\end{tabular}

Danach haben die ausländischen Gesellschaften nur in der großen Todesfallversicherung einen nennenswerten Betrag in Rückdeckung gegeben. Aus der kleinen . Versicherung haben sie im Gegensatze zu den deutschen Gesellschaften überhaupt nichts rückversichert, weil bei ihnen Quotenrückversicherungsverträge nicht vorkommen. In Rückdeckung übernommen haben die ausländischen Gesellschaften an deutschen Versicherungen nach den dem Aufsichtsamte vorgelegten Nachweisungen nur $2455604 \mathrm{Nl}$. in der Todesfallversicherung und $200 \mathrm{~d}$. Jahresrente in der Rentenversicherung. Es ist 
aber nicht ausgeschlossen, daß dieser Betrag zu klein ist, denn wie es den Anschein hat, ist die Auffassung darüber, was von dem inclirekten Geschäft als deutsches zu bezeichnen ist, noch keine gleichmäßige.

Über das Verhältnis der mit Gewinnbeteiligung der Versicherten abgeschlossenen Verträge zu den überhaupt abgeschlossenen ergibt sich bei der Beschränkung auf das direkte Geschäft, daß

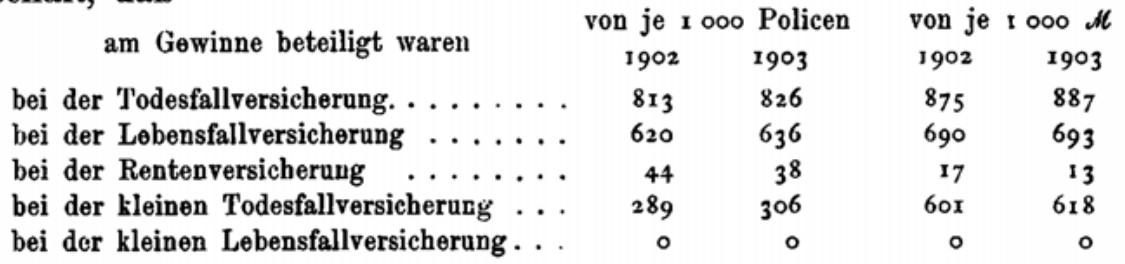

Die Beteiligung am Gewinne war hiernach bei den ausländischen Gesellschaften bei allen Versicherungsarten, mit alleiniger Ausnahme der Rentenversicherung, Ende ${ }_{1903}$ ziemlich viel beträchtlicher als Ende 1902, was sich nur zum Teil dadurch erklärt, daß in dem Berichtsjahre die Mutual eingeschlossen werden konnte. Wie bei den deutschen Gesellschaften ist auch bei den ausländischen Unternehmungen die Gewinnbeteiligung der Versicherten bei der Todesfallversicherung am häufigsten. Das vorjährige Ergebnis, daß Todesfallversicherungen mit Gewinnbeteiligung bei den ausländischen Gesellschaften, obwohl bei ihnen die Aktiengesellschaften nicht von den Gegenseitigkeitsvereinen getrennt sind, noch seltener seien als bei den deutschen Aktiengesellschaften, läßt sich nach den diesjährigen Erfahrungen nur noch hinsichtlich der Versicherungssummen, nicht auch hinsichtlich der Policenzahl aufrecht erhalten.

In der Volksversicherung sind bei uns aus dem Auslande nur Aktiengesellschaften vertreten; es ist also ein Vergleich der obigen Zahlen mit denen der deutschen Aktiengesellschaften ohne weiteres zulässig, und ein solcher führt zu dem Ergebnisse, daß die ausländischen Gesellschaften bei uns verhältnismäßig sehr viel weniger Volksversicherungen mit Gewinnbeteiligung erwerben als die deutschen Unternehmungen.

Die durchschnittliche Versicherungssumme oder Jahresrente einer selbst abgeschlossenen Versicherung stellt sich für das deutsche Geschäft der ausländischen Gesellschaften auf folgende Beträge:

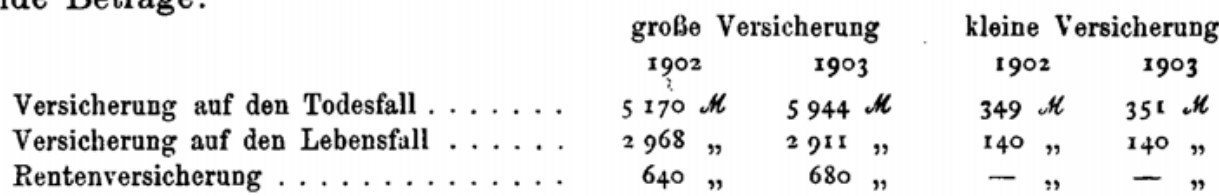

Diese Zahlen sind für die große Versicherung nicht unbeträchtlich größer als die entsprechenden, die oben für die Versicherung der deutschen Unternehmungen angeführt sind. In der kleinen Versicherung ist der Unterschied nur bei der Lebensfallversicherung von größerem Belang; die ausländischen Gesellschaften weisen hier einen sehr niedrigen Durchschnittssatz auf.

\section{Die Bewegung in dem Versicherungsbestand im Jahre 1903.}

Die Bewegung in dem Versicherungsbestande hat in diesem Jahre etwas ausführlicher und richtiger dargestellt werden können als in der vorjährigen Statistik, weil die Zerlegung der überhaupt abgeschlossenen Versicherungen nach unmittelbarem und mittelbarem Geschäfte sowohl für den Schluß des Vorjahrs wie auch für den Schluß des Berichtsjahrs vorgenommen werden konnte, was im Vorjahre nicht angängig war. Die Tabellen geben jetzt den Bestand Ende 1902, den Bestand Ende 1903 und den reinen Zugang nicht nur für die überhaupt übernommenen Versicherungen, sondern daneben auch für die selbst abgeschlossenen und für die in Rückdeckung übernommenen Versicherungen; 
sie behandeln ferner den Abgang an selbst abgeschlossenen Versicherungen insofern eingehender als im Vorjahr, als der vorzeitige Abgang zerlegt ist in Abgang durch Rückkauf, durch Verfall, Verzicht, Nichteinlösung der Police und durch Unwandlung oder Übertragung: endlich sind die auf die verschiedenen Ursachen des Abganges bezüglichen Zahlen zu dem mittleren Bestand an selbst abgeschlossenen Versicherungen ins Verhältnis gesetzt und nicht, wie aus dem eben erwähnten Grunde im Vorjahre geschehen mußte, zu dem mittleren Bestand an überhaupt übernommenen Versicherungen.

Die Bewegung in dem Versicherungsbestande der deutschen Unternehmungen, die Deutsche allen Kreisen der Bevölkerung offen stehen, ist in den Tabellen I 21 bis I 27 ausführlich allgemeine behandelt. Dabei hat aber, wie schon oben bemerkt ist, die Trennung zwischen großer mungen und kleiner Versicherung nicht so vollständig durchgeführt werden können wie bei der Beschreibung des Bestandes am Ende des Berichtsjahrs, insbesondere war die Bewegung der Sterbegeldversicherung bei einigen Unternehmungen nicht aus der der großen Versicherung auszuscheiden.

Die folgende C̈bersicht gibt zunächst einen Überblick über die Bewegung der wichtigsten Versicherungsarten in dem gesamten Geschäfte der großen deutschen Unternehmungen, dabei ist bei der Volksversicherung und bei der sonstigen kleinen Versicherung die Todesfall- mit der Lebensfallversicherung zusammengefaßt. In Betracht gezogen sind nur die selbst abgeschlossenen Versicherungen.

Bewegung im Versicherungsbestande der deutschen Unternehmungen

\begin{tabular}{|c|c|c|c|c|c|c|c|c|c|c|}
\hline \multirow{7}{*}{$\begin{array}{l}\text { Bestand Ende } 1902 \\
\text { Zugang . . . . . . } \\
\text { Abgang . . . . . } \\
\text { Reiner Zugang . } \\
\text { Bestand Ende } 1903\end{array}$} & \multicolumn{2}{|c|}{$\begin{array}{c}\text { Versicherung } \\
\text { auf } \\
\text { den T'odesfall } \\
\text { Policen } 1000 ، \mathbb{H}\end{array}$} & \multicolumn{2}{|c|}{$\begin{array}{c}\text { Versicherung } \\
\text { auf } \\
\text { den Lebensfall } \\
\text { Policen } 1000 \mathrm{~J}\end{array}$} & \multicolumn{2}{|c|}{$\begin{array}{c}\text { Renten- } \\
\text { versicherung } \\
\text { Policen } 1000\end{array}$} & \multicolumn{2}{|c|}{$\begin{array}{c}\text { Volks- } \\
\text { versicherung } \\
\text { Policen } 1000 \mathscr{M}\end{array}$} & \multicolumn{2}{|c|}{$\begin{array}{l}\text { Sonstige kleine } \\
\text { Versicherung } \\
\text { Policen } 1000 \text { A }\end{array}$} \\
\hline & \multicolumn{8}{|c|}{$\begin{array}{l}\text { Aktiengesellschaften } \\
26 \text { Gesellschaften } \mid 23 \text { Gesellschaften| \& Gesells }\end{array}$} & \multicolumn{2}{|c|}{4 Gesellschaften } \\
\hline & 1020509 & 3465385 & $23^{1} 3^{8} 9$ & 408826 & 26023 & 12368 & 3562617 & 631545 & 5619 & 2640 \\
\hline & $13814 x$ & 421719 & 36319 & 53996 & 2926 & 1475 & 801852 & 157956 & I 973 & 922 \\
\hline & 78895 & 204614 & 19131 & 34802 & I 293 & 588 & 366214 & 85346 & 927 & 413 \\
\hline & 59246 & 1) 217088 & $17 \times 88$ & 19194 & 1633 & 887 & 435638 & 72610 & $x 046$ & 509 \\
\hline & $|\mathrm{x} 079755|$ & 3682473 & 248577 & 428020 & 27656 & 13255 & $|3998255|$ & 704155 & 6665 & $3 \times 49$ \\
\hline \multicolumn{11}{|c|}{ Gegenseitigkeitsvereine } \\
\hline & \multicolumn{2}{|c|}{20 Vereine } & $18 \mathrm{Ve}$ & reine & $12 \mathrm{Ver}$ & reine & \multicolumn{2}{|c|}{3 Vereine } & \multicolumn{2}{|c|}{3 Vereine } \\
\hline Bestand Ende 1902 & 670619 & 3428583 & 315693 & 456307 & 22316 & 4084 & 146521 & $26 \times 55$ & $2296 r$ & 7242 \\
\hline Zugang ........ & 51697 & 276104 & 15849 & 27679 & I 164 & 406 & 34204 & 6235 & $5^{87}$ & 255 \\
\hline 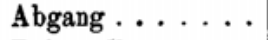 & 29908 & 140426 & $2 \times 151$. & 30595 & 1039 & 169 & $23 \times 33$ & 4847 & 1054 & 247 \\
\hline Reiner Zugang . . & 21789 & r 35678 & -5302 & -2916 & 125 & 237 & $1107 x$ & × 388 & -467 & 8 \\
\hline Bestand Ende 1903 & 692408 & 3564261 & 310391 & 453391 & 22441 & 4321 & 157592 & 27543 & 22494 & 7250 \\
\hline \multicolumn{11}{|c|}{ Sämtliche Unternehmungen } \\
\hline & \multicolumn{2}{|c|}{\begin{tabular}{l|}
47 Unter- \\
nehmungen
\end{tabular}} & \multicolumn{2}{|c|}{\begin{tabular}{|}
44 Unter- \\
nehmungen
\end{tabular}} & \multicolumn{2}{|c|}{\begin{tabular}{|}
35 Unter- \\
nehmungen
\end{tabular}} & \multicolumn{2}{|c|}{$\begin{array}{l}11 \text { Unter- } \\
\text { nehmungen }\end{array}$} & \multicolumn{2}{|c|}{$\begin{array}{l}7 \text { Unter- } \\
\text { nehmungen }\end{array}$} \\
\hline Bestand Ende 1902 & 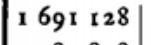 & 6893968 & 547082 & $865 \times 33$ & $4^{8} 339$ & $1645^{2}$ & 3709138 & 657700 & 28580 & 9882 \\
\hline Zugang . . . . . & 189838 & 697823 & $52 \times 68$ & 81675 & 4090 & I $88 \mathrm{x}$ & 836056 & $164 r 9 x$ & 2560 & $1 \mathrm{I} 77$ \\
\hline 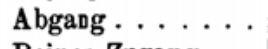 & 108803 & 345040 & 40282 & 65397. & 2332 & 757 & 389347 & 90193 & I 981 & 660 \\
\hline Reiner Zugang . . & 81035 & 352766 & II 886 & $\times 6278$ & I $75^{8}$ & 1124 & 446709 & 73998 & 579 & 517 \\
\hline Bestand Ende 1903 & $|1772 \times 63|$ & 7246734 & $55^{8} 968$ & 881411 & 50097 & I7576 & $4 \times 55847$ & $73 \times 698$ & 29159 & 10399 \\
\hline
\end{tabular}


Man ersieht hieraus, daß ein Rückgang des Geschäfts nur bei den Gegenseitigkeitsvereinen in der Versicherung auf den Lebensfall und in der ..sonstigen kleinen Versicherung" und hier auch nur in der Zahl der Policen zu verzeichnen ist, daß diese Abnahme des Geschäfts aber vollständig überdeckt wird durch die Zunahme, die bei den Aktiengesellschaften auch bei diesen Versicherungsarten eingetreten ist, so daß sich als Gesamtergebnis für alle Unternehmungen ein Anwachsen sowohl der Zahl der Policen als auch der Versicherungssumme herausstellt.

Des näheren ist bei den einzelnen Versicherungsarten folgendes zu bemerken:

In der Versicherung auf den Todesfall sind beim unmittelbaren Geschäft 189838 Policen und 697,823 Millionen Mark oder 11,2 und 10,1 Prozent des Bestandes zu Anfang des Jahres zugegangen, davon kommen 179458 Policen (94,5 Prozent) über 661,898 Millionen Mark (94,9 Prozent) auf neue Abschlüsse; abgegangen sind 108803 Policen über 345,040 Millionen Mark oder 6,3 und 4,9 Prozent des mittleren Bestandes des Berichtsjahrs. Der reine Zugang hat 81035 Policen und 352,766 Millionen Mark oder 4,8 und 5,1 Prozent des anfänglichen Bestandes betragen.

Bei den in Rückdeckung übernommenen Versicherungen hat bei der Gesamtheit der Unternehmungen die Zahl der Policen um 95 abgenommen, die Versicherungssumme dagegen um 4,283 Millionen Mark zugenommen.

In der Versicherung auf den Lebensfall sind bein unmittelbaren Geschäft 52168 Policen und 81,675 Millionen Mark oder 9,5 und 9,4 Prozent des Bestandes zu Anfang des Jahres zugegangen, davon kommen 51157 Policen (98,1 Prozent) über 80,192 Millionen Mark (98,2 Prozent) auf neue Abschlüsse; abgegangen sind 40282 Policen über 65,,397 Millionen Mark oder 7,3 und 7,j Prozent des mittleren Bestandes des Berichtsjahrs. Der reine Zugang hat 11886 Policen und 16,278 Millionen Mark oder 2,2 und 1,9 Prozent des anfänglichen Bestandes betragen.

Bei den in Rückdeckung übernommenen Versicherungen hat bei den Aktiengesellschaften die Zahl der Policen nur um fünf und die Versicherungssumme um 0,063 Millionen Mark zugenommen; bei den Gegenseitigkeitsvereinen sind keine Versicherungen in Rückdeckung übernommen worden.

In der Rentenversicherung sind beim unmittelbaren Geschäfte 4090 Policen und 1.881 Millionen Mark oder 8,5 und 11,4 Prozent des Bestandes zu Anfang des Jahres zugegangen, davon kommen 3885 Policen (95,0 Prozent) über 1.812 Millionen Mark (96,3 Prozent) auf neue Abschlüsse; abgegangen sind 2332 Policen über 0,757 Millionen Mark oder 4,7 und 2,2 Prozent des mittleren Bestandes des Berichtsjahrs. Der reine Zugang hat 1758 Policen und 1,124 Millionen Mark oder 3,6 und 6,8 Prozent des anfänglichen Bestandes betragen.

Bei den in Rückdeckung übernommenen Versicherungen hat bei der Gesamtheit der Unternehmungen die Zahl der Policen um fünf abgenommen, die Versicherungssumme dagegen um 0,008 Millionen Mark zugenommen.

In der Volksversicherung sind 836056 Policen und 164,191 Millionen Mark oder 22,5 und 25,0 Prozent des Bestandes zu Anfang des Jahres zugegangen, davon kommen 800397 Policen (95,7 Prozent) über 156,535 Millionen Mark (95,3 Prozent) auf neue Abschlüsse; abgegangen sind 389347 Policen über 90,193 Millionen Mark oder 10,0 und 6,5 Prozent des mittleren Bestandes des Berichtsjahrs. Der reine Zugang hat 446709 Policen und 73,998 Millionen Mark oder 12,0 und 11,3 Prozent des anfänglichen Bestandes betragen.

Bei den in Rückdeckung übernommenen Versicherungen hat bei den Aktiengesellschaften die Zahl der Policen um 88 und die Versicherungssumme um 0,017 Millionen Mark abgenommen; bei den Gegenseitigkeitsvereinen sind keine Versicherungen in Rückdeckung übernommen worden.

Wegen der großen Bedeutung, die den verschiedenen Ursachen des Abgangs von Versicherungen zukommt, sind in unseren Tabellen für die Hauptversicherungsarten nicht nur die absoluten Zahlen für die Häufigkeit der einzelnen Crsachen angegeben, sondern 
es ist auch berechnet, wie viel von 1000 Policen oder von $1000 \mathrm{dt}$ Versicherungssumme des durchschnittlichen Bestandes durch Tod, wie viel durch Ablauf usw. in Wegfall gekommen sind. Für einen allgemeinen Überblick wird es genügen, die Ursachen des Wegfalls hier in drei Gruppen zusammenzufassen: ordentlicher Abgang (Abgang durch Tod oder Ablauf), Storno mit Vergütung (Abgang durch Rückkauf, Reduktion, Umwandlung oder C'bertragung) und Storno ohne Vergütung (Abgang durch Verfall, Verzicht, Nichteinlösung der Police).

\begin{tabular}{|c|c|c|c|c|c|c|}
\hline \multirow[b]{2}{*}{ Versicherung auf den Todesfall } & \multicolumn{2}{|c|}{ ordentlicher Abgang } & \multicolumn{2}{|c|}{ Storno mit Vergütung } & \multicolumn{2}{|c|}{ Storno ohne Vergütung } \\
\hline & Policen & Summen & Policen & Summen & Policen & Summen \\
\hline Aktiengesellschaften. . . . & 18 & 17 & II & 13 & 46 & 27 \\
\hline Gegenseitigkeitsvereine . . . & 19 & 18 & 12 & 12 & $x_{3}$ & 10 \\
\hline Versicherung auf den Lebensfall & & & & & & \\
\hline Aktiengesellschaften . . . . . & 29 & $3 \mathrm{I}$ & 22 & 27 & 29 & 25 \\
\hline Gegenseitigkeitsvereine ... & 42 & 38 & 13 & 16 & 12 & 13 \\
\hline Rentenversicherung & & & & & & \\
\hline Aktiengesellschaften.... & 39 & 37 & 6 & 6 & 3 & 3 \\
\hline Gegenseitigkeitsvereine . . . & 39 & $3^{1}$ & 7 & 8 & $\mathbf{I}$ & 1 \\
\hline Volksversicherung & & & & & & \\
\hline Aktiengesellschaften . . . . & 15 & $x_{4}$ & 2 & $\mathrm{I}_{4}$ & 80 & 99 \\
\hline Gegenseitigkeitsvereine . . . & 12 & 13 & 3 & 14 & 136 & 153 \\
\hline
\end{tabular}

An diesen Zahlen wird $u$. a. beachtenswert sein, daß bei der Volksversicherung ein höherer Satz der Versicherungssummen als der Policenzahl durch Storno ohne Entschädigung ausscheidet und daß bei der großen Todesfallversicherung das Entgegengesetzte statt hat. Bei der Volksversicherung verfallen danach mehr verhältnismäßig hohe, bei der großen Versicherung mehr verhältnismäßig niedrige Versicherungen.

Die außerordentlich kleinen Zahlen für den vorzeitigen Abgang bei der Rentenversicherung finden ihren einfachen Grund darin, daß es sich hier in der überwiegenden Zahl der Fälle um sofort beginnende Renten gegen einmalige Prämien handelt; ein vorzeitiger Abgang kann also nur bei den verhältnismäßig seltenen Versicherungen von aufgeschobenen Renten gegen Jahresprämien vorkommen.

Wie man auch die Bewegung beurteilen mag, nach dem Zugange, dem Abgang oder dem reinen Zuwachs, immer zeigt sie sich in der Volksversicherung verhältnismäßig am größten. Ebenso steht hinsichtlich der vorzeitigen Auflösung der Versicherungsverträge ohne Vergütung die Volksversicherung an der Spitze.

Von einer Betrachtung der Bewegung in der Invaliditätsversicherung wird hier mit Rücksicht auf die Ungenauigkeit der betreffenden Angaben abgesehen. Was die Krankenversicherung anlangt, so mag es genügen anzuführen, daß bei den diesen Zweig betreibenden Unternehmungen der ganze Versicherungsbestand von 5072 Versicherungen über $21346 \mathscr{A C}$ Krankengeld pro Tag am Ende des Vorjahrs auf 9601 Versicherungen über 32549 At Krankengeld pro Tag am Ende des Berichtsjahrs gestiegen ist.

Wenn man bei den Versicherungseinrichtungen der größeren Berufsvereinigungen ver(Pensionskassen und Sterbekassen) diejenigen außer Betracht läßt, bei welchen die Ver- ${ }_{\text {einchichtun- }}^{\text {sichos- }}$ sicherungssummen nicht angegeben werden können, weil sie veränderlich sind, dann gen von erhält man folgendes Bild:

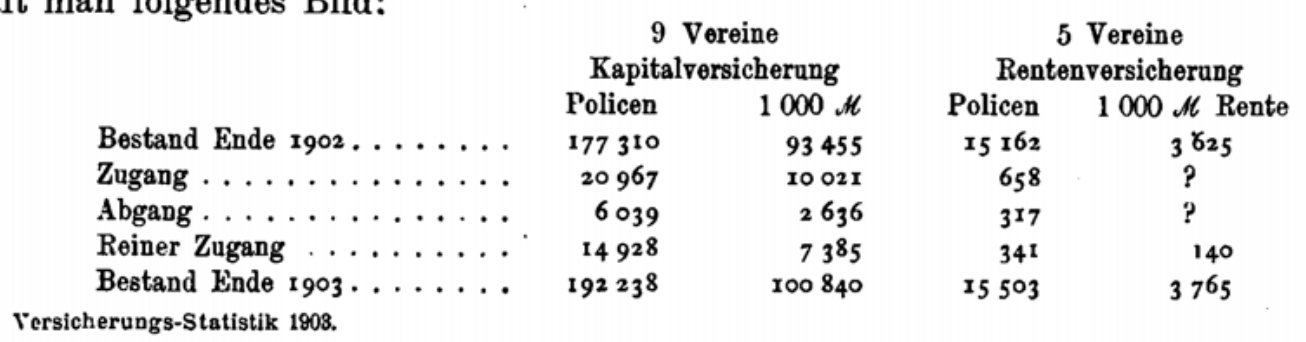


Die Zunahme in der Kapitalversicherung (Todesfall- und Lebensfallversicherung) hat danach bei den Policen 8,4 und bei den Versicherungssummen 7.9 Prozent des Bestandes zu Anfang des Jahres betragen. Von dem Zugange kommen 20743 Policen (99,0 Prozent) über 9,922 Millionen Mark (99,0 Prozent) auf neue Abschlüsse; ausgeschieden sind durch ordentlichen Abgang von den Policen 13 und von den Versicherungssummen ebenfalls 13 Promille des durchschnittlichen Bestandes, durch Storno mit Vergütung von den Policen 1 und von den Summen 2 vom Tausend, durch Storno ohne Vergütung von den Policen 19 und von den Summen 12 vom Tausend. Der reine Zugang belief sich bei den Policen auf 8,1 Prozent und bei den Summen auf 7,9 Prozent des anfänglichen Bestandes.

In der Rentenversicherung sind bei den Policen 4,3 Prozent des Bestandes zu Anfang des Jahres zugegangen; von dem Zugange kommen 637 Policen oder 96,8 Prozent auf neue Abschlüsse. Der Abgang belief sich auf 2,1 Prozent des mittleren Bestandes. Der reine Zugang machte bei den Policen 2,2 Prozent und bei den Summen der Jahresrenten 3,9 Prozent aus.

Die Bewegung in dem deutschen Versicherungsbestande der in dieser Statistik behandelten ausländischen Lebensversicherungsunternehmungen ist in den Tabellen I 29 bis I 32 ausführlich dargestellt. Aus diesen Tabellen ist folgende Übersicht hergeleitet:

Bewegung im deutschen Versicherungsbestand ausländischer Gesellschaften

\begin{tabular}{|c|c|c|c|c|c|c|c|c|}
\hline * & \multicolumn{2}{|c|}{$\begin{array}{c}\text { Versicherung auf den } \\
\text { Todesfall } \\
21 \text { Gesellschaften }\end{array}$} & \multicolumn{2}{|c|}{$\begin{array}{l}\text { Versicherung auf den } \\
\text { Lebensfall } \\
21 \text { Gesellschaften }\end{array}$} & \multicolumn{2}{|c|}{$\begin{array}{c}\text { Renten- } \\
\text { versicherung } \\
18 \text { Gesellschaften }\end{array}$} & \multicolumn{2}{|c|}{$\begin{array}{c}\text { Volks- } \\
\text { versicherung } \\
3 \text { Gesellschaften }\end{array}$} \\
\hline Bestand Ende rgor $\ldots \ldots \ldots$ & ro4 738 & 594750 & 40429 & 121491 & I 493 & 999 & II 205 & 3625 \\
\hline Zugang $\ldots \ldots \ldots \ldots$ & 13088 & ror 310 & 5 OI 3 & 10833 & 149 & 112 & I 423 & 544 \\
\hline Abgang $\ldots \ldots \ldots \ldots$ & 7726 & $44 \circ 16$ & 3097 & 9074 & 56 & 32 & I 584 & 488 \\
\hline Reiner Zugang . . . . . . . . & 5362 & 57294 & I 916 & I 759 & 93 & 80 & $-16 r$ & 56 \\
\hline Bestand Ende $1903 \ldots \ldots \ldots$ & 110100 & 652044 & 42345 & 123250 & I $5^{86}$ & I 079 & II 044 & $368 r$ \\
\hline
\end{tabular}

Danach hat das deutsche Geschäft der ausländischen Gesellschaften auch im Jahre $19 \circ 3$ überall Fortschritte gemacht, nur in der Volksversicherung ist die Zahl der Policen ein wenig zurückgegangen. Im besonderen sind in der großen Versicherung auf den Todesfall im unmittelbaren Geschäfte 13088 Policen und 101,310 Millionen Mark oder 12,z und 17,0 Prozent des Bestandes zu Anfang des Jahres zugegangen, davon kommèn 12758 Policen über 97,253 Millionen Mark (96,0 Prozent) auf neue Abschlüsse; abgegangen sind 7726 Policen über 44,016 Millionen Mark oder 7,2 und 3, standes des Berichtsjahrs. Der reine Zugang hat 5362 Policen und 57,294 Millionen Mark oder 5,1 und 9,6 Prozent des anfänglichen Bestandes betragen.

Bei den in Rückdeckung übernommenen Versicherungen hat die Zahl der Policen um 28 und die Versicherungssumme um 0,247 Millionen Mark zugenommen.

In der Versicherung a uf den Lebensfall sind 5013 Policen und 10,833 Millionen Mark oder 12,3 und 8,9 Prozent des Bestandes zu Anfang des Jahres zugegangen, davon kommen 4933 Policen über 10,745 Millionen Mark (99,2 Prozent) auf neue Abschlüsse; abgegangen sind 3097 Policen über 9,074 Millionen Mark oder 7,5 und 7,4 Prozent des mittleren Bestandes des Berichtsjahrs. Der reine Zugang hat 1916 Policen und 1,759 Millionen Mark oder 4,7 und 1,4 Prozent des anfänglichen Bestandes betragen.

In Rückdeckung sind Versicherungen auf den Lebensfall nicht übernommen worden. 
In der Rentenversicherung sind 149 Policen und 0,112 Millionen Mark oder 10,0 und 11,2 Prozent des Bestandes zu Anfang des Jahres zugegangen, davon kommen 144 Policen über 0,108 Millionen Mark (82,4 Prozent) auf neue Abschlüsse; abgegangen sind 56 Policen üher 0,032 Millionen Mark oder 3,6 und 3,1 Prozent des mittleren Bestandes des Berichtsjahrs. Der reine Zugang hat 93 Policen und 0,080 Millionen Mark oder 6,2 und 8,0 Prozent des anfänglichen Bestandes betragen.

Der Bestand der in Rückdeckung übernommenen Rentenversicherungen hat sich gegen das Vorjahr nicht geändert.

In der Volksversicherung sind 1423 Policen und 0, öt Millionen Mark oder 12,7 und 15,0 Prozent des Bestandes zu Anfang des Jahres zugegangen, davon kommen 1377 Policen über 0,542 Millionen Mark (99,6 Prozent) auf neue Abschlüsse; abgegangen sind 1584 Policen über 0,488 Millionen Mark oder 14,2 und 13,4 Prozent des mittleren Bestandes des Berichtsjahrs. Der reine Zugang hat - 161 Policen und 0,056 Millionen Mark oder 1,t und 1,5 Prozent des anfänglichen Bestandes betragen.

In Rückdeckung übernommene Volksversicherungen sind nicht vorgekommen.

Die Verhältniszahlen für den Zugang in der großen Versicherung sind hiernach überall bei den ausländischen Unternehmungen größer als bei den inländischen, für den Zugang in der Volksversicherung gilt das Entgegengesetzte.

Über die Häufigkeit der verschiedenen Ursachen des Abganges seien hier die folgenden Zahlen angeführt, die den oben für die deutschen Unternehmungen angegebenen entsprechen.

Abgang in Tausendsteln des durchschnittlichen Bestandes

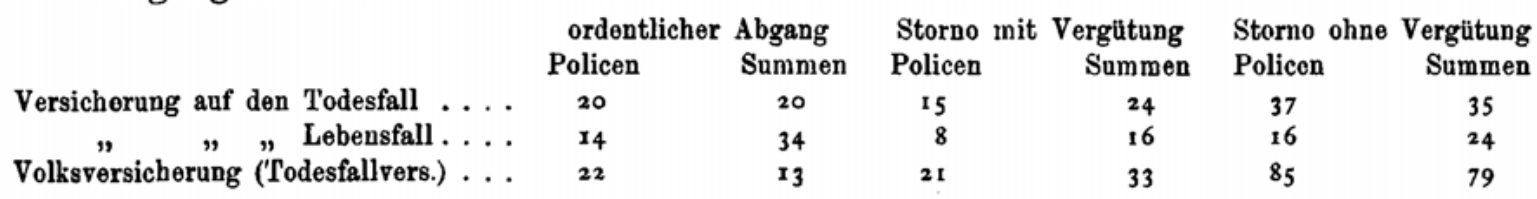

Unterschiede, wie sie sich bei den deutschen Unternehmungen zwischen den Policen und Summen zeigten, scheinen hier nicht zu bestehen.

\section{Die Betriebsrechnung für 1903.}

Die Gewinn- und Verlustrechnung, wie sie in den Tabellen I 33, 36, 38 und 40 gegeben ist, unterscheidet sich von den Aufstellungen, welche die Unternehmungen nach dem Formulare L 1 der Rechnungsvorschriften dem Aufsichtsamt einzureichen haben, dadurch, daß die veränderlichen Fonds (Prämienreserve, Prämienüberträge, Reserven für schwebende Versicherungsfälle, Gewinnreserven und sonstige Reserven) nicht mit ihren Beständen zu Anfang des Jahres in Einnahme und mit ihren Werten am Ende des Jahres in Ausgabe gestellt sind, sondern daß nur ihre Änderungen durch den Jahresbetrieb verbucht sind; denn nur diese beeinflussen das Ergebnis der Jahresrechnung; außerdem wird die Rechnung durchsichtiger. Der Stand der Fonds am Ende des Jahres findet sich in der Bilanz. Sodann ist der Vortrag aus dem Überschusse des Vorjahrs hier fortgelassen worden,- um das Ergebnis des Geschäftsbetriebs lediglich aus dem Berichtsjahre zu erhalten.

Die Betriebsrechnung bezieht sich auf das ganze (deutsche und ausländische) Lebensversicherungsgeschäft der Unternehmungen. Für die Volksversicherung ist bei den deutschen Gesellschaften eine besondere Gewinn- und Verlustrechnung (Tabelle I 36) aufgestellt; die sonstige kleine Versicherung und bei den ausländischen Gesellschaften auch die Volksversicherung ist mit dem Geschäft in der großen Versicherung zusammen verrechnet (Tabelle I 33 und 40). Auch sind beim Atlas und bei der Wilhelma die geringen Einnahmen und Ausgaben der Kautionsversicherung hier mitverbucht (vgl. die Anmerkungen 4 und $5 \mathrm{zu}$ Tabelle I 34 S. 109). 
Die in den Übersichten über die Gewinn- und Verlustrechnung nur summarisch eingestellten Prämien und Vergütungen der Rückversicherer einerseits und die Zahlungen für selbstabgeschlossene und für in Rückdeckung übernommene Versicherungen sowie die Rückversicherungsprämien andererseits sind in den Tabellen I 34, 37 und 39 erläutert. Dentsche
allgemeine
Onter-
nesondere Gewinn- und Verlustrechnung aufgestellt, es hat daher auch hier nur das
nehmungen
gesamte Geschäft nachgewiesen werden können, und zwar ist das der Providentia bei
Gruppe I und das der Thuringia bei Gruppe IV gegeben. Dabei ist jedoch so verfahren, daß
in Tabelle I 33 bei den Einnahmen aus Prämien, Policegebühren, Vergütungen der Rück-
versicherer sowie aus dem Unterschiede zwischen der Schadenreserve und den Zahlungen
und Rückstellungen für Versicherungsfälle der Vorjahre und bei den Ausgaben für Ver-
sicherungsleistungen, für Rückversicherung und für Vermehrung der Prämienreserve nur die
aus der Lebensversicherung herrührenden Posten eingestellt sind. Bei der Providentia sind
die aus den übrigen Geschäftszweigen stammenden entsprechenden Beträge, die bei den
Sondereinnahmen und Sonderausgaben der Gruppen II und IV erläutert sind, bei den
sonstigen Einnahmen und sonstigen Ausgaben in Tabelle I 33 verrechnet, bei der'Thuringia
dagegen sind die in Tabelle I 33 für die Lebensversicherung angegebenen Einnahmen und
Ausgaben in der bei Gruppe IV gegebenen Gewinn- und Verlustrechnung in der Summe
der Sondereinnahmen einerseits und in der Summe der Sonderausgaben andererseits
enthalten. betragen:

Bei Ausschluß der Thuringia haben in der Lebensversicherung ohne Volksversicherung

\begin{tabular}{|c|c|c|c|}
\hline 501. & $\begin{array}{l}\text { bei der Gesamtheit } \\
\text { der Unternehmungen }\end{array}$ & $\begin{array}{l}\text { bei } 26 \text { Aktien- } \\
\text { gesellschaften }\end{array}$ & $\begin{array}{l}\text { bei } 21 \text { Gegen- } \\
\text { seitigkeitsvereinen }\end{array}$ \\
\hline $\begin{array}{l}\text { die Summe der Einnabmen } \ldots \ldots \\
\text { die Summe der Ausgaben } \ldots \ldots \ldots\end{array}$ & $\begin{array}{l}473671399 M \\
400723986 \leadsto\end{array}$ & $\begin{array}{l}254211831 \mu \\
221516848, \mu\end{array}$ & $\begin{array}{l}219459568 \mu \\
179207138 \mu\end{array}$ \\
\hline der Jahresüberschuß & $72947413 \mu$ & $32694983 \mu$ & $40252430 \mu$ \\
\hline
\end{tabular}

Von den gesamten Einnahmen entfallen bei den Aktiengesellschaften $179041421 \mathscr{M}$ oder 70,4 Prozent auf Prämien und Policegebühren, $8053035 \mathscr{A}$ oder 3,2 Prozent auf Vergütungen der Rückversicherer, 529724 N․ oder 0,2 Prozent auf Verminderung der sonstigen Reserven $53375829 \mathscr{M}$ oder 21,0 Prozent auf Ertrag und Gewinn aus den Kapitalanlagen und 13211822 \% oder 5,2 Prozent auf sonstige Einnahmen; von den gesamten Ausgaben entfallen 89837541 Nh oder 40,6 Prozent auf Versicherungsleistungen, 88285337 Nl oder 39,8 Prozent auf Vermehrung der Prämienreserven, 8237773 oder 3,7 Prozent auf Rückversicherungsprämien, $4930159 \mathscr{M}$ oder 2,2 Prozent auf Vermehrung der sonstigen Reserven (Spezialreserven) $26456643 \mathscr{M}$ oder 11,9 Prozent auf Verwaltungskosten und Steuern, 435955 , Ml oder 0,2 Prozent auf Abschreibungen und Verluste aus Kapitalanlagen und endlich $3333430, \|$ oder 1, 5 Prozent auf sonstige Ausgaben.

Bei den Gegenseitigkeitsvereinen kommen von den gesamten Einnahmen $155586852 \mathbb{A l}$ oder 70,9 Prozent auf Prämien und Policegebühren, 534748 $\mathscr{M}$ oder 0,3 Prozent auf Vergütungen der Rückversicherer, $232559 \mathscr{M}$ oder 0,1 Prozent auf Verminderung der Spezialreserven, 56208919 AN oder 25,6 Prozent auf Ertrag oder Gewinn aus Kapitalanlagen und $6827442 \mathscr{M}$ auf sonstige Einnahmen; von den gesamten Ausgaben kommen $89344486 \mathscr{M}$ oder 49,9 Prozent auf Versicherungsleistungen, $65105110 \mathscr{A l}$ oder 36,3 Prozent auf Vermehrung der Prämienreserven, $962439 \mathscr{M}$ oder 0,5 Prozent auf Rückversicherungsprämien, $2581149 \mathscr{M}$ oder 1,4 Prozent auf Vermehrung der Spezialreserven, 14550107 chl oder 8,1 Prozent auf Verwaltungskosten und Steuern, 440803 AN oder 0,2 Prozent auf Abschreibungen und Verluste aus Kapitalanlagen, $393223 \mathscr{H}$ oder 0,2 Prozent auf Vergütungen für übernommene Rückversicherungen und endlich 6223044 \% oder 6,4 Prozent auf sonstige Ausgaben. 
Das Geschäftsjahr 1903 hat einer der 26 Aktiengesellschaften im großen Versicherungsgeschäft einen Verlust von 435846 . von zusammen $33130829 \cdot \mathscr{K}$ gebracht; drei der 21 Gegenseitigkeitsvereine haben einen Verlust von zusammen 82729 $\mathscr{N}$ erlitten und 17 einen Gewinn von $40335159 \mathrm{Nl}$ erzielt. Von dem durch die Bilanz ausgewiesenen Gewinn und dessen Verteilung wird unten gesprochen werden.

Was die kleine Versicherung betrifft, so ergeben sich dafür nach Ausschluß des bei Gruppe II mit nachgewiesenen Geschäfts des Nordstern, Unfall- und Alters-VersicherungsAktiengesellschaft, also für die Volksversicherung im Sinne dieser Statistik folgende Zahlen:

\begin{tabular}{|c|c|c|c|}
\hline Volksversicherung & Gesamtheit & 9 Aktiengesellschaften & $\begin{array}{c}3 \text { Gegenseitigkeits } \\
\text { vereine }\end{array}$ \\
\hline r Ein & $64759295 \cdot N$ & $62506785 \mu$ & $2252510 M$ \\
\hline umme der Ausgabe & 53992082, & $52040136 "$ & I 951946 \\
\hline Jahresüberschuß $\ldots$ & $10767213 \mu$ & го $466649 M$ & $300564 N$ \\
\hline
\end{tabular}

Von den gesamten Einnahmen kornmen bei den Aktiengesellschaften 54371142 Al oder 87,1 Prozent auf Prämien und Policegebühren, 252602 , $/ 1$ oder 0,4 Prozent auf Vergütungen der Rückversicherer und $7788125 \mathscr{N}$ oder 12,5 Prozent auf Ertrag und Gewinn aus Kapitalanlagen: von den gesamten Ausgaben kommen 8483922 M oder 16,3 Prozent auf Versicherungsleistungen, 27490141 , Nh oder 52,8 Prozent auf Vermehrung der Prämienreserve, $286167 \mathscr{H}$ oder 0,5 Prozent auf Rüekversicherungsprämien, 1592183 cN oder 3,1 Prozent auf Vermehrung der Spezialreserven, $13965175 \mathscr{M}$ oder 26,8 Prozent auf Verwaltungskosten und Steuern und 127072 a'll oder 0,2 Prozent auf Abschreibungen und Verlust aus Kapitalanlagen.

Bei den Gegenseitigkeitsvereinen kommen von den gesamten Einnahmen $1975431 \mathcal{M}$ oder 87, 7 Prozent auf Prämien- und Policegebühren und $271110 \mathscr{M}$. oder 12,0 Prozent auf Ertrag und Gewinn aus Kapitalanlagen; Vergütungen der Rückversicherer sind nicht eingenommen; von den gesamten Ausgaben entfallen $293376 \mathscr{M}$ oder 15,0 Prozent auf Versicherungsleistungen, $965495 \mathscr{W}$ oder 49,j Prozent auf Vermehrung der Prämienreserve, $4385 \mathscr{M}$ oder 0,2 Prozent auf Rückversicherungsprämien, $8269 \mathscr{M}$ oder 0,4 Prozent auf Vermehrung der Spezialveserven, 650502 , N oder 33,3 Prozent auf Verwaltungskosten und Steuern und endlich 29054 , $/$ oder 1,5 Prozent auf Abschreibungen und Kursverlust.

Von den 9 Aktiengesellschaften, deren Betriebsrechnung für das Geschäft in der Volksversicherung in Tabelle I 36 aufgestellt ist, hat im Jahre 1903 eine mit einem Verlust von 25284 \& gearbeitet, an dem Gewinne von $10401933 \mathscr{N}$. sind 8 von ihnen beteiligt. Von den 3 Gegenseitigkeitsvereinen haben zwei einen $\ddot{\text { berschuß }}$ von zusammen 300564 ، $/$ erzielt, ein Verein hat weder einen Gewinn noch einen Verlust nachgewiesen, indem er den geringfügigen Überschuß bereits auf Abschreibung früherer Verluste verrechnet hat.

Stellt man aus den Tabellen I 34 und 37 die Hauptzahlen zusammen, so erhält man folgende Angaben für das Lebensversicherungsgeschäft aller deutschen allgemeinen Versicherungsunternehmungen, wobei die Kriegs- und Extraprämien mit den Prämien für selbst abgeschlossene Versicherungen bezw. mit den Rückversicherungsprämien vereinigt worden sind, weil sie nicht überall in befriedigender Weise ausgeschieden werden konnten. (Tabelle umstehend.)

Von den Ansprüchen auf Versicherungsleistungen aus selbst abgeschlossenen Versicherungen wurden im Berichtsjabr in der großen Versicherung von der Gesamtheit der Unternehmungen 94,0 Prozent des Wertes befriedigt und 6,0 Prozent zurückgestellt, im besondern von den Aktiengesellschaften 96,1 und 3,9 Prozent, von den Gegenseitigkeitsvereinen 91,9 und 8,1 Prozent; in der Volkversicherung wurden von der Gesamtheit der Unter- 


\begin{tabular}{|c|c|c|c|c|c|c|c|c|c|}
\hline \multirow[b]{2}{*}{ Im Jahre 1903 haben betragen } & \multicolumn{3}{|c|}{$\begin{array}{l}\text { in der großen Ver- } \\
\text { sicherung }\end{array}$} & \multicolumn{3}{|c|}{$\begin{array}{c}\text { in der } \\
\text { Volksversicherung }\end{array}$} & \multicolumn{3}{|c|}{$\begin{array}{c}\text { in der sonstigen kleinen } \\
\text { Versicherung }\end{array}$} \\
\hline & \begin{tabular}{|c|} 
bei der \\
Ge- \\
sant- \\
heit von \\
48 \\
Unter- \\
neh- \\
mungen
\end{tabular} & \begin{tabular}{|c|} 
bei 27 \\
Aktien- \\
gesell- \\
schaf- \\
ten
\end{tabular} & $\begin{array}{c}\text { bei } 21 \\
\text { Gegen- } \\
\text { seitig- } \\
\text { keits- } \\
\text { ver- } \\
\text { einen }\end{array}$ & \begin{tabular}{|c|} 
boi der \\
Ge- \\
samt- \\
heit von \\
11 \\
Unter- \\
neh- \\
mungen
\end{tabular} & $\begin{array}{c}\text { bei } 8 \\
\text { Aktien- } \\
\text { gesell- } \\
\text { schaf- } \\
\text { ten }\end{array}$ & $\begin{array}{c}\text { bei } 3 \\
\text { Gegen- } \\
\text { seitig- } \\
\text { keits- } \\
\text { ver- } \\
\text { einen }\end{array}$ & \begin{tabular}{|c|} 
bei der \\
Ge- \\
samt- \\
heit von \\
13 \\
Unter- \\
neh- \\
mungen
\end{tabular} & \begin{tabular}{|c|} 
bei 9 \\
Aktien- \\
gesell- \\
schaf- \\
ten
\end{tabular} & $\begin{array}{l}\text { bei } 4 \\
\text { Gegen- } \\
\text { seitig- } \\
\text { keits- } \\
\text { ver- } \\
\text { einen }\end{array}$ \\
\hline 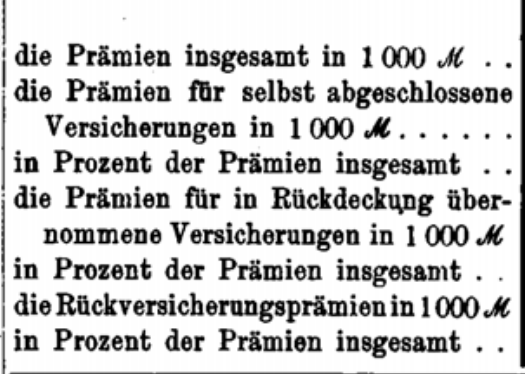 & $\begin{array}{c}33^{8} 149 \\
334226 \\
98,8 \\
3923 \\
1,2 \\
9209 \\
2,7\end{array}$ & $\begin{array}{c}183032 \\
179525 \\
98,1 \\
\\
3507 \\
1,9 \\
8247 \\
4,5\end{array}$ & $\begin{array}{c}155 \times 17 \\
154701 \\
99,7 \\
4 \times 6 \\
0,3 \\
962 \\
0,6\end{array}$ & \begin{tabular}{c|}
55809 \\
55802 \\
100,0 \\
7 \\
7 \\
0,013 \\
290 \\
0,5
\end{tabular} & \begin{tabular}{c|}
53859 \\
53852 \\
100,0 \\
\\
7 \\
0,013 \\
286 \\
0,5
\end{tabular} & $\begin{array}{c}1950 \\
1950 \\
100,0\end{array}$ & \begin{tabular}{c|c|}
1 & 333 \\
1 & 253 \\
$94: 0$ & \\
80 \\
6,0 \\
52 \\
3,9
\end{tabular} & \begin{tabular}{cc|}
1 & 105 \\
1 & 025 \\
92,8 & \\
80 \\
7,2 \\
52 \\
4,7
\end{tabular} & $\begin{array}{r}228 \\
228 \\
100,0 \\
- \\
- \\
- \\
-\end{array}$ \\
\hline $\begin{array}{l}\text { die Versicherangsleistungen insgesamt } \\
\text { in } 1000 \AA \ldots \ldots \ldots \ldots \ldots \ldots \ldots \\
\text { die Leistangen für selbst abgeschlossene } \\
\text { Versicherungen in } 1000 \ldots \ldots \ldots \ldots\end{array}$ & $\begin{array}{r}182411 \\
178624\end{array}$ & $\begin{array}{l}93290 \\
89896\end{array}$ & $\begin{array}{l}89121 \\
88728\end{array}$ & $\begin{array}{l}8777 \\
8774\end{array}$ & $\begin{array}{l}8484 \\
848 x\end{array}$ & 293 & 317 & 245 & 223 \\
\hline $\begin{array}{r}\text { insgessmt . . . . . . . . . . . . . . . } \\
\text { die Leistungen für in Rückdeckung über- }\end{array}$ & 97,9 & 96,4 & 99,6 & 100,0 & roo,o & 100,0 & 67,7 & 38,4 & 100,0 \\
\hline $\begin{array}{r}\text { nommene Versicherungen in } 1000 \mu \\
\text { in Prozent der Versicherungsleistungen }\end{array}$ & 3787 & 3394 & 393 & 3 & 3 & - & $x 51$ & $15 x$ & - \\
\hline die Vergütungen der Rückversicherer & $2, \mathbf{r}$ & 3,6 & $\circ, 4$ & 0,034 & 0,035 & - & 32,3 & $6 r, 6$ & - \\
\hline $\begin{array}{l}\text { in } 1000 \mu \ldots \ldots \ldots \ldots \ldots \\
\text { in Prozent der Versicherungsleistungen }\end{array}$ & 8604 & 8069 & 535 & 253 & 253 & - & 44 & 44 & - \\
\hline insgesamt. $\ldots \ldots \ldots \ldots \ldots$ & 4,7 & 8,6 & 0,6 & 2,9 & 3,0 & - & 9,4 & 18,0 & - \\
\hline
\end{tabular}

nehmungen 99,5 Prozent gezahlt und 0,5 Prozent zurückgestellt, im besondern von den Aktiengesellschaften 99,5 und 0,5 Prozent, von den Gegenseitigkeitsvereinen 97,8 und 2,2 Prozent.

Ver- Über die Gewinn- und Verlustrechnung der in diese Statistik aufgenommenen Vereinriohtun- sicherungseinrichtungen der größeren Berufsvereinigungen geben die Tabellen I 38 und gen von I 39 Aufschluß. Daraus sind folgende Zahlen zu entnehmen:

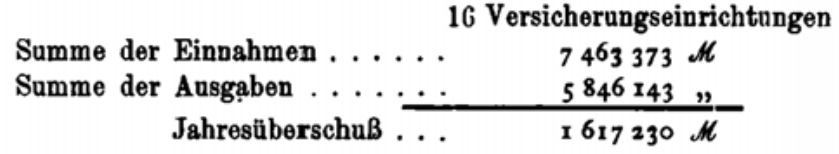

Von den gesamten Einnahmen kommen $5848154 \mathscr{M}$ oder 78,4 Prozent auf Prämien und Policegebühren (Eintrittsgelder), $51642 \mathscr{M}$ oder 0,7 Prozent auf Vergütungen der Rückversicherer, $42481 \mathscr{M}$ oder 0,6 Prozent auf Verminderung der sonstigen Reserven und $1447088 \mathscr{M}$ oder 19,4 Prozent auf Ertrag und Gewinn aus Kapitalanlagen; von den gesamten Ausgaben kommen $1753301 \mathscr{M}$ oder 30,0 Prozent auf Versicherungsleistungen, 3337607 $\mathscr{H}$ oder 57,1 Prozent auf Vermehrung der Prämienreserven, $63121 \mathscr{H}$ oder

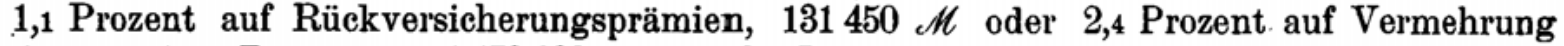
der sonstigen Reserven und $479689 \mathscr{M}$ oder 8,2 Prozent auf Verwaltungskosten und Steuern. 
Zur Beurteilung des Geschäftsbetriebs derjenigen ausländischen Lebensversicherungsgesellschaften, über welche diese Statistik berichtet, ist in Tabelle I 40 die Gewinn- und Verlustrechnung für das ganze in- und ausländische Geschäft möglichst nach denselben Grundsätzen dargestellt, die bei den deutschen Gesellschaften zur Anwendung gekommen sind. Daneben sind aber in Tabelle I 41 die Einnahmen und Ausgaben der ausländischen Gesellschaften allein aus dem deutschen Geschäfte zusammengestellt. Danach haben betragen die Einnahmen an Prämien im deutschen Geschäft insgesamt $32566383 \mathrm{~N}$. Davon kommen 32382605 , N. oder 99,4 Prozent auf selbst abgeschlossene Versicherungen und $183778 \mathscr{M}$ oder 0,6 Prozent auf in Rückdeckung übernommene Versicherungen. Die Rückversicherungsprämien beliefen sich auf $962489 \mathscr{N}$, sodaß von der gesamten Prämieneinnahme 3,0 Prozent für Rückversicherung wieder ausgegeben wurden. Für Versicherungsleistungen waren bereit zu stellen insgesamt $17028176 \mathscr{M}$, davon $16898733 \mathfrak{N}$ oder 99,2 Prozent für selbst abgeschlossene Versicherungen und 129443 Al oder 0,8 Prozent für in Rückdeckung übernommene Versicherungen. An Vergütungen der Rückversicherer sind 671513 . 11 eingenommen, das sind 3,9 Prozent der gesamten für Versicherungsleistungen bereit $\mathrm{zu}$ haltenden Summen.

\section{Die Bilanz für den Schlufs des Jahres 1903.}

Die Bilanz ist in dieser Statistik grundsätzlich für das Gesamtgeschäft einer jeden Gesellschaft aufgestellt und bei derjenigen Gruppe nachgewiesen, zu der das Unternehmen seinem Hauptbetriebe nach gehört.

Dementsprechend sind in Tabelle I 42 die Bilanzen für 26 Aktiengesellschaften und Deutsohe 21 Gegenseitigkeitsvereine aufgeführt; faßt man sie zusammen, so schließt die Gesamt- ${ }^{\text {allgemein }}$ Unter bilanz für Ende ${ }_{1903}$ bei" den Aktiengesellschaften mit $1783980901 \mathcal{N}$ und bei den nehmungen Gegenseitigkeitsvereinen mit 1448424457 / ab; die Hauptposten stehen darin mit folgenden Beträgen:

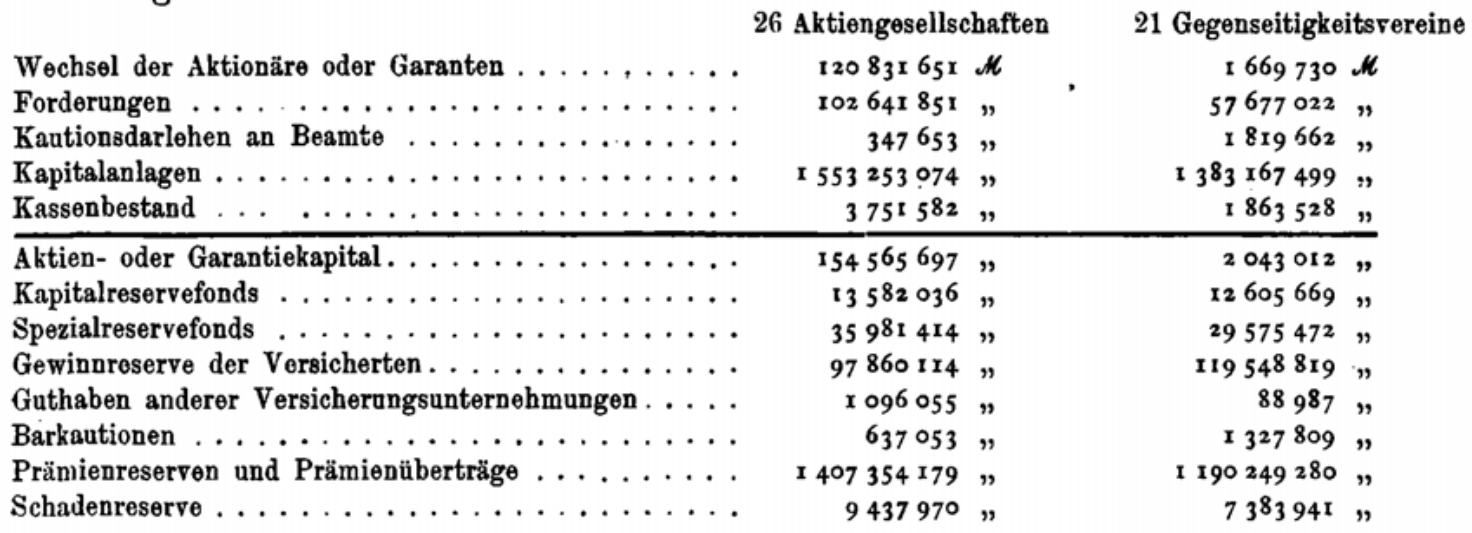

Bei den Aktiengesellschaften kommen nach den vorstehenden Zahlen

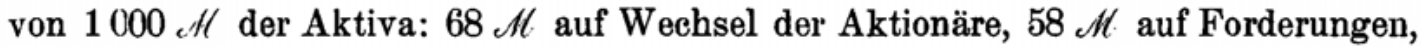
0,20 th auf Kautionsdarlehen an Beamte, $871 \mathrm{cH}$ auf Kapitalanlagen und $2 \mathscr{M}$ auf Kassenbestand;

von $1000 \mathscr{H}$ der Passiva: $87 \mathscr{M}$. auf Aktienkapital, $8 \mathscr{H}$ auf Kapitalreserve, $20 \mathscr{N}$ auf Spezialreserven, $55 \mathscr{M}$ auf Gewinnreserve der Versicherten, 0,61 $\mathscr{M}$ auf Guthaben anderer Versicherungsunternehmungen, 0,36 $\mathscr{M}$ auf Barkautionen, 789 , 1 auf Prämienreserve und Prämienüberträge und 5 \% auf Schadenreserve. 
Bei den Gegenseitigkeitsvereinen kommen dagegen

von 1000 , N/ der Aktiva: 1 . /l auf Wechsel der Garanten, 40 d/ auf Forderungen, $1 . / 1$ auf Kautionsdarlehen an Beamte, 955 , /l auf Kapitalanlagen und 1 , $/$ suf Kassenbestand;

von $1000 \mathrm{dH}$ der Passiva: $1 \mathrm{~N}$ auf Garantiekapital, 7 , N auf Kapitalreserve, $20 \mathrm{dH}$ auf Spezialreserven, $83 \mathscr{N}$ auf Gewinnreserve der Versicherten, 0,06 $\mathscr{M}$ auf Guthaben anderer Versicherungsunternehmungen, 1 d auf Barkautionen, $822 \mathrm{~d}$ auf Prämienreserve und Prämienüberträge und $5 \mathrm{Ml}$ auf Schadenreserve.

Die Kapitalanlagen (Tabelle I 43) verteilen sich auf folgende Wertarten:

\begin{tabular}{|c|c|c|c|c|c|c|c|}
\hline \multirow{3}{*}{ Grundbesitz $\ldots \ldots \ldots \ldots \ldots$} & \multicolumn{3}{|c|}{26 Aktiengesellschaften } & \multicolumn{4}{|c|}{21 Gegenseitigkeitsvereine } \\
\hline & 51510500 & $\mathscr{M}$ oder & $3,3 \%$ & 15262170 & $\mathscr{M}$ & oder & $\mathbf{x}, \mathbf{1} \%$ \\
\hline & I 325420408 & 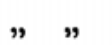 & 85,4, & t 171987714 & $"$ & $"$ & 84,7, \\
\hline Darlehen auf Wertpapiere ...... & 495818 & $"$ & 0,0, & I 123988 & $"$ & $"$ & 0,1, \\
\hline Wertpapiere .......... & 67870142 & $n$ & 4,4, & 98095324 & $"$ & $"$ & $7,1 \Rightarrow$ \\
\hline Policedarlehen $\ldots \ldots \ldots \ldots \ldots$ & 107632817 & $"$ & 6,9, & 92465969 & $"$ & $"$ & 6,7, \\
\hline Wechsel . . . . . . . . . & 323389 & $"$ & $\circ, 0 "$ & 4223855 & $"$ & $"$ & 0,3, \\
\hline Sonstige Anlagen $\ldots \ldots \ldots \ldots$ & - & & - & 8479 & $n$ & $"$ & - \\
\hline
\end{tabular}

Im Vergleiche mit dem Vorjahre hat bei den Aktiengesellschaften zugenommen der Grundbesitz um $838670 \mathscr{H}$, der Bestand an Hypotheken um $139660827 \mathscr{N}$, der an Wertpapieren um $3489055 \mathscr{M}$ und der an Policedarlehen um $9971055 \mathscr{M}$, dagegen haben abgenommen die Darlehen auf Wertpapiere um $809082^{\circ} \mathscr{H}$. und die Wechsel um $1083729 \mathscr{M}$. - Bei den Gegenseitigkeitsvereinen hat zugenommen der Besitz an Hypotheken um $64523390 \mathscr{H}$, an Wertpapieren um $2533143 \mathscr{M}$, an Policedarlehen um $4005215 \mathscr{M}$. und an Wechseln um $1488295 \mathscr{N}$, dagegen steht der Grundbesitz Ende r903 mit 491751 \% weniger zu Buche und anderweite Kapitalanlagen waren Ende 1903 nicht mehr vorhanden. Nimmt man an, daß sich der Verkehr in Grundbesitz, in Hypotheken, in Wertpapieren und Wechseln auf dem öffentlichen Kapitalmarkt abgewickelt hat, so sind diesem Markte allein durch die allgemeinen deutschen Lebensversicherungsunternehmungen im Jahre 1903 netto 210,95 Millionen Mark zugeführt worden, und davon sind 0,35 Millionen Mark in 'Grundstücken, 204,18 Millionen Mark in Hypotheken, 6,02 Millionen Mark in Wertpapieren und 0,40 Millionen Mark in Wechseln angelegt worden.

Die Forderungen bestehen:

\begin{tabular}{|c|c|c|c|c|c|c|c|c|}
\hline \multirow{5}{*}{ 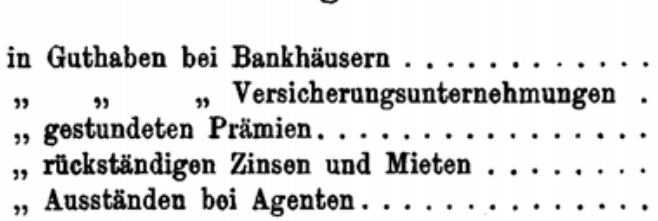 } & \multicolumn{4}{|c|}{26 Aktiengesellschaften } & \multicolumn{4}{|c|}{21 Gegenseitigkeitsvereine } \\
\hline & \multirow{4}{*}{$\begin{array}{r}41701977 \\
2520691 \\
37820597 \\
6112368 \\
14486218\end{array}$} & \multirow{3}{*}{$\begin{array}{l}\text { oder } \\
" " \\
" \\
"\end{array}$} & \multirow{3}{*}{\multicolumn{2}{|c|}{$\begin{array}{rl}40,6 & \% \\
2,5 & " \\
36,8 & " \\
6,0 & "\end{array}$}} & \multicolumn{3}{|c|}{$13035421 M$ oder } & \multirow[t]{2}{*}{$\begin{aligned} 22,6 & \% \\
0,8 & \%\end{aligned}$} \\
\hline & & & & & \multirow{2}{*}{$\begin{array}{r}463817 \\
24070557 \\
10742778\end{array}$} & \multirow{2}{*}{$"$} & \multirow{3}{*}{$"$} & \\
\hline & & & & & & & & 18,7 \\
\hline & & & 14,1 & $"$ & 9364449 & $"$ & & 16,2 \\
\hline
\end{tabular}

Die Prämienreserven und die Prämienüberträge sind in Tabelle I 44 nach Versicherungsarten auseinander gezogen. Sie bedürfen indessen noch folgender Erläuterungen. Nach $\S 58$ des Versicherungsaufsichtsgesetzes soll bei Rückversicherungen die Prämienreserve von dem rückversicherten Unternehmen berechnet, aufbewahrt und verwaltet werden. Die Rechnungsvorschriften bestimmen, daß als Prämienreserve in der Gewinnund Verlustrechnung und in der Bilanz nur die in eigener Verwaltung befindlichen $\mathrm{Be}-$ träge geführt werden sollen. Für alle bis zum Inkrafttreten des Gesetzes abgeschlossenen Versicherungen sollen die Prämienreserven unter Berücksichtigung der geltenden Rückversicherungsverträge eingestellt werden. Bei den nach dem Inkrafttreten des Gesetzes abgeschlossenen Versicherungen sollen die Prämienreserven für alle selbst abgeschlossenen Versicherungen einschließlich der hiervon in Rückdeckung gegebenen Summen in dem 
Kapitel Prämienreserve nachgewiesen werden; für die in Rückdeckung übernommenen Versicherungen aber sollen die Prämienreserven, soweit sie abzugeben sind, in der Gewinnund Verlustrechnung bei den Vergütungen für in Rückdeckung übernommene Versicherungen verrechnet werden und also in der Bilanz nicht bei den Prämienreserven erscheinen. Diese Vorschriften haben bei der Rechnungslegung für $190_{3}$ mehrere Gesellschaften überhaupt noch nicht beachtet, andere haben die Prämienreserven in fremder Verwaltung ganz aus der Rechnung fortgelassen, und wieder andere haben diese Posten bei anderen Kapiteln behandelt. Da das Formular L 3 der Rechnungsvorschriften die Mittel bietet, den Betrag der bei Rückversicherungen abgegebenen Reserven festzustellen, so sind diese Beträge, abweichend von der vorjährigen Statistik, jetzt auch bei denjenigen Gesellschaften, die sie noch mit durch die Bilanz geführt haben, abgesetzt worden. Dieses Verfahren entspricht nicht nur den Rechnungsvorschriften, sondern verhindert auch, daß die Reserven für solche Versicherungen, die von einer unter Reichsaufsicht stehenden Gesellschaft bei einer ebensolchen Gesellschaft in Rückdeckung gegeben sind, mehrfach aufgeführt werden. Eine Folge dieses Vorgehens ist übrigens, daß die Guthaben bei Versicherungsunternehmungen jetzt eine weniger bedeutende Rolle spielen als in früheren Bilanzen; die jetzt, gegrebenen Zahlen entsprechen in weit höherem Maße den tatsächlichen Verhältnissen.

Die Nachweisung der Prämienreserven und der Prämienüberträge erfolgt bei den verschiedenen Anstalten und bei versehiedenen Versicherungsarten nicht nach denselben Grundsätzen, soda $\beta$ die als Prämienreserven oder Prämienüberträge angegebenen Summen weder die reinen Prämienreserven noch die reinen Überträge darstellen. Sie sollen deshalb hier zusammengefaßt werden.

In Tabelle I 44 sind für alle deutschen Aktiengesellschaften Prämienreserven und Prämienüberträge in eigener Verwaltung in Höhe von 1447,760 Millionen Mark nachgewiesen. Setzt man davon diejenigen Beträge ab, die in den Spalten 13 und 14 für Unfall- und Haftpflichtversicherungen und in den Anmerkungen S. 153 für die anderen Versicherungszweige mit insgesamt 48,471 Millionen Mark aufgeführt und in den Spalten 6 und 11 mit enthalten sind, so erhält man als Prämienreserven nebst Überträgen allein für die Lebensversicherung 1399,289 Millionen Mark. Bei den Gegenseitigkeitsvereinen gehören die in der Tabelle aufgeführten Beträge von zusammen 1 202,566 Millionen Mark vollständig der Lebensversicherung an. Die gesamte Prämienreserve für Lebens- und Krankenversicherung stellt sich danach für alle deutschen allgemeinen Unternehmungen auf 2601,855 Millionen Mark, wovon 53,8 Prozent auf die Aktiengesellschaften und 46,2 Prozent auf die großen Gegenseitigkeitsvereine kommen. In welchem Maße die einzelnen Versicherungsarten beteiligt sind, ist aus nachstehender Übersicht zu entnehmen:

\begin{tabular}{|c|c|c|c|c|c|c|}
\hline \multirow{3}{*}{ Versicherungsart } & \multicolumn{6}{|c|}{ Prämienreserven und Prämienüberträge } \\
\hline & \multicolumn{2}{|c|}{$\begin{array}{l}\text { bei der Gesamtheit } \\
\text { der Unternehmungen }\end{array}$} & \multicolumn{2}{|c|}{$\begin{array}{l}\text { bei den Aktien- } \\
\text { gesellschaften }\end{array}$} & \multicolumn{2}{|c|}{$\begin{array}{l}\text { bei den Gegen- } \\
\text { seitigkeitsvereinen }\end{array}$} \\
\hline & $1000 \cdot n$ & $\%$ & $1000 \mathrm{Ak}$ & ${ }^{0} i_{0}$ & $1000 \mu$ & $\%$ \\
\hline Versicherung auf den Todesfall . . . . . . . . & 2057347 & $79, \mathbf{1}$ & I II9422 & 80,0 & 937926 & 78,0 \\
\hline$" \quad, \quad "$ Lebensfall . . . . . . . & $357 \times 34$ & 13,7 & 164288 & 11,7 & 192846 & 16,0 \\
\hline 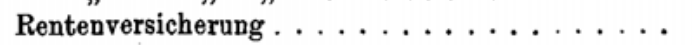 & 184740 & $7, \mathrm{t}$ & $1 \times 3489$ & $8, \mathrm{x}$ & 71251 & 5,9 \\
\hline Sonstige Versicherungen. . . . . . . . . . & 2634 & $0, \mathbf{r}$ & 2090 & 0,2 & 544 & $0, \mathbf{1}$ \\
\hline Zusammen ... & 2601855 & 100,0 & 1399289 ! & 100,0 & I 202567 & 100,0 \\
\hline
\end{tabular}

Die Verteilung des Gewinns ist in Tabelle I 45 nitgeteilt. Wenn dabei die Summe des Gesamtgewinns nicht mit der Summe der Jahresüberschüsse und der Vorträge aus dem Vorjahre, wie sie in der Bilanz (Tab. I 42) enthalten sind, übereinstimmt, so erklärt sich das folgendermaßen. Beim Janus in Hamburg wird satzungsgemäß nicht der in der 
Bilanz ausgewiesene Gewinn verteilt, sondern es wird dieser Gewinn zu den noch nicht verteilten Überschüssen der Vorjahre hinzugerechnet und dann von dieser Sumne $1 / 3$ für das Geschäftsjahr verteilt, während $2 / 3$ weiter reserviert werden. Ferner ist bei den Gegenseitigkeitsvereinen auch die Verteilung des vom Allgemeinen Deutschen Versicherungsverein in Stuttgart aus dem Lebensversicherungsgeschäft erzielten Gewinns mit nachgewiesen, während die Verteilung des aus den anderen Versicherungszweigen hervorgegangenen Gewinns bei Gruppe II gegeben ist.

Sonach standen für die Gewinnverteilung zur Verfügung bei den Aktiengesellschaften $48103579 \mathrm{M}$. und bei den Gegenseitigkeitsvereinen $40635723 \mathrm{di}$ und diese Beträge sind folgendermaßen verteilt worden:

\begin{tabular}{|c|c|c|c|c|c|c|c|}
\hline & \multicolumn{3}{|c|}{ Aktiengesellschaften } & \multicolumn{4}{|c|}{ Gegenseitigkeitsvereine } \\
\hline & 253856 & At oder & $0,5 \%$ & 924239 & $\mathscr{M}$ & oder & $2,3^{0}: 0$ \\
\hline An den Kapitalreservefonds. . . . . . & 1932601 & $"$ & 4,0, & 2158426 & $"$ & " & 5,2, \\
\hline$"$ den Beamtenfonds . . . . & 301180 & $"$, & 0,6, & 122903 & $"$ & $"$ & 0,3, \\
\hline " die Aktionäre oder Garanten . . . . . . & $466 \times 409$ & $"$ & $9,7 \%$ & 27303 & $"$ & $"$ & 0,7, \\
\hline 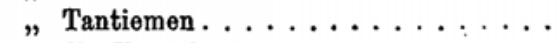 & I 607373 & $"$ & $3,3 "$ & 368757 & $"$ & $"$ & $\circ, 9$, \\
\hline " die Versicherten ........... & 37368460 & $"$ & 77,7, & $37025 \times 37$ & $"$ & $"$ & $9 \mathrm{r}, \mathrm{r}$, \\
\hline , anderweit $\ldots \ldots \ldots \ldots \ldots$ & I 805000 & 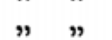 & 3,8, & 8958 & 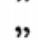 & $"$ & 0,2, \\
\hline , den Vortrag auf neue Rechnung ... & 173700 & $"$, & ০,4 ", & - & & & - \\
\hline
\end{tabular}

Die Kapitalreserve aller Unternehmungen steht in der diesjährigen Bilanz höher als in der vorjährigen, bei den Aktiengesellschaften um $252374 \mathscr{N}$, bei den Gegenseitigkeitsvereinen um $294953 \mathrm{~A}$; die Spezialreserven sind bei den Aktiengesellschaften um $3930093 \mathscr{H}$. gewachsen und bei den Gegenseitigkeitsvereinen um $1066245 \mathscr{M}$, endlich die Gewinnreserven der Versicherten um 12992319 , Nh und $7661650 \mathrm{NH}$.

Die Bilanzen der Versicherungseinrichtungen von Berufsvereinigungen mit den zugeeisrrichtun- hörigen Erläuterungen sind in den Tabellen I 48 bis I 52 gegeben. Die Gesamtbilanz gen von schließt ab mit $43394664 \mathscr{M}$ und ihre Hauptposten weisen folgende Beträge auf:

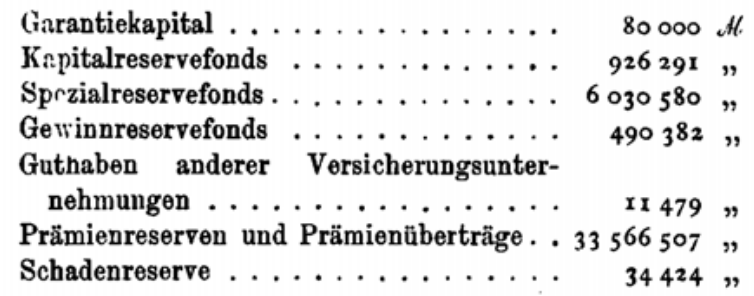

Es kommen also von $1000 \mathscr{M}$ der Aktiva $21 \mathscr{M}$ auf Forderungen, $870 \mathscr{H}$ auf Kapitalanlagen und 0,59 $\mathscr{M}$ auf Kassenbestand; von $1000, \mathcal{H}$ der Passiva 2 ell auf Garantiekapital, $21 \mathcal{M}$. auf Kapitalreservefonds, $139 \mathrm{dt}$ auf Spezialreservefonds, 11 He auf Gewinnreserve der Versicherten, 0,26 $\mathscr{M}$ auf Guthaben anderer Versicherungsunternehmungen, 774 , 11 . auf Prämienreserve und Prämienüberträge und schließlich 0,79 All auf Schadenreserve.

Von den insgesamt $37761252 \mathscr{H}$. betragenden Kapitalanlagen kommen 474093 1,2 Prozent auf Grundbesitz, $31439092 \mathscr{M}$ oder 83,3 Prozent auf Hypotheken, $5769456 \mathscr{H}$ oder 15,3 Prozent auf Wertpapiere und der Rest von $78611 \mathscr{H}$. oder 0,2 Prozent auf Policedärlehen. Die Vergleichung des Bestandes der in der vorjährigen Statistik behandelten Unternehmungen am Ende des Vorjahrs mit dem am Ende des Berichtsjahrs ergibt eine Zunahme des Grundbesitzes um $96678 \mathscr{N}$, des Hypothekenbesitzes um 5135199 Nh und der Policedarlehen um $21384 \mathscr{N}$; der Bestand an Wertpapieren hat um $1178263 \mathscr{N}$ abgenommen.

Die Aktivforderungen $(874858 \mathscr{M})$ setzen sich zusammen aus $249263 \mathscr{M}$ oder 28,5 Prozent Guthaben bei Bankhäusern, 26179 NH oder 3,0 Prozent Guthaben bei anderen Versicherungsunternehmungen, 247078 « oder 28,2 Prozent gestundeten Prämien, 
234048 , / oder 26,8 Prozent rückständigen Zinsen und Mieten, 115492 , /l oder 13,2 Prozent Ausständen bei Agenten und 2798, 11 oder 0,3 Prozent sonstigen Forderungen.

Die Bilanzen von dreizehn Anstalten von Berufsvereinigungen weisen einen Gewinn von zusammen 1732522 dt auf, der, wie folgt, verteilt worden ist: 293758 , /l oder 17,0 Prozent an den Kapitalreservefonds, 1062175 dl oder 61,3 Prozent an die Spezialreserven, 5822 sit oder 0,3 Prozent an die Beamtenfonds, 15777 dl oder 0,9 Prozent Tantiemen, 294160, /l oder 17,0 Prozent an die Versicherten, 60034 /l oder 3,5 Prozent Vortrag auf neue Rechnung und 796 , /l oder 0,04 Prozent anderweit.

Der Kapitalreservefonds ist gegen die vorjährige Bilanz um 125306 ، , die Spezialreserven sind um $1124360 \mathrm{~d}$ und die Gewinnreserven um $100146 \mathrm{dl}$ gewachsen.

Die Bilanzen von 21 ausländischen Gesellschaften, deren Lebensversicherungsgeschäfte Die ausin dieser Statistik behandelt sind, finden sich in Tabelle I כ̋3; von einer weiteren, der Uändischen Northern Assurance Company, ist die Bilanz bei der Feuerversicherung in Tabelle IV 29 mungen mitgeteilt. Die Bilanzen sind nach Möglichkeit den für die Bilanzen der deutschen Unternehmungen geltenden Grundsätzen angepaßt worden.

Von besonderem Interesse ist die Art der Kapitalanlagen (Tabelle I 54); deshalb sind in der folgenden Übersicht die in der Tabelle 154 aufgeführten ausländischen Gesellschaften nach Heimatländern zusammengefaßt und zum Vergleich die Verhältniszahlen für die deutschen Unternehmungen wiederholt:

Von je 1000 dl Kapitalanlagen entfielen auf

\begin{tabular}{|c|c|c|c|c|c|c|c|c|}
\hline \multicolumn{2}{|l|}{ bei } & $\begin{array}{l}\text { Grund- } \\
\text { besitz }\end{array}$ & $\begin{array}{l}\text { Hypo- } \\
\text { theken }\end{array}$ & $\begin{array}{l}\text { Darlehen } \\
\text { auf Wert- } \\
\text { papiere }\end{array}$ & $\begin{array}{l}\text { Wert- } \\
\text { papiere }\end{array}$ & $\begin{array}{l}\text { Police- } \\
\text { darlehen }\end{array}$ & Wechsel & $\begin{array}{c}\text { Gesamtbetrag } \\
\text { der Kapital- } \\
\text { anlagen } \\
\text { I } 000 M\end{array}$ \\
\hline \multicolumn{2}{|c|}{3 amerikanischen Gesellschaften } & 70 & 178 & 27 & 652 & 73 & - & 3039110 \\
\hline \multicolumn{2}{|l|}{2 dänischen } & 23 & 602 & - & 276 & 99 & - & 26686 \\
\hline \multirow{2}{*}{\multicolumn{2}{|c|}{$\begin{array}{l}1 \text { onglischen Gesellschaft. . . . . } \\
2 \text { französischen Gesellschaften . . }\end{array}$}} & 93 & 198 & $\mathbf{I}$ & 574 & 134 & - & 32732 \\
\hline & & t9o & 73 & I & 707 & 29 & - & 309670 \\
\hline \multicolumn{2}{|c|}{3 niederländischen $\quad, \quad \ldots$} & 174 & 296 & 24 & $44 \mathrm{I}$ & 65 & - & 97461 \\
\hline & . & 82 & 275 & 4 & $55^{6}$ & 75 & 8 & 424180 \\
\hline $\begin{array}{l}3 \text { schweizerischen } \\
\text { dagegen bei }\end{array}$ & $"$ & 52 & 674 & 4 & 211 & 53 & 6 & 123499 \\
\hline \multirow{2}{*}{\multicolumn{2}{|c|}{$\begin{array}{r}26 \text { deutschen Aktiengesellschaften } \\
21 \quad, \quad \text { Gegenseitigkeitsver- } \\
\text { einen........ }\end{array}$}} & 33 & 854 & - & 44 & 69 & - & I 553253 \\
\hline & & II & 847 & $\mathbf{I}$ & 71 & 67 & 3 & I 383167 \\
\hline Berufs & fsvereinigungen & 12 & 833 & - & 153 & 2 & - & $3776 r$ \\
\hline
\end{tabular}

Von den allgemeinen deutschen Unternehmungen haben die Kapitalversicherung im Das ausAuslande mittelbar oder unmittelbar betrieben je 15 in den Niederlanden und in Österreich ländische Auslante mittelbar oder unmittelbar betrieben je 15 in den Niederlanden und in Usterreich, Geschätt 14 in der Schweiz, 12 in Belgien, 11 in Dänemark, je 6 in Frankreich und Schweden, je der 4 in Finnland, Italien, Norwegen und Rußland, 3 in Ungarn, je 2 in Luxemburg und in deutschen der Türkei und endlich je 1 in Ägypten, Griechenland, Portugal und Spanien. In der ne'mungen Rentenversicherung ist das Geschäft natürlich auch im Auslande weniger verbreitet, es haben gearbeitet 7 Gesellschaften in der Schweiz, je 5 in Belgien, Dänemark, in den Niederlanden und in Österreich, 3 in Schweden, 2 in Frankreich und je 1 in Finnland, Italien, Luxemburg und Norwegen.

In der Kapitalversicherung hatten die deutschen Unternehmungen Ende r903 im Auslande 152949 Verträge über 553,172 Millionen Mark laufen, das sind 7,1 Prozent aller ihrer Versicherungen und 6,8 Prozent ihrer gesamten Versicherungssummen; die vereinnahmten Prämien, einschließ]ich der davon für Rückversicherungen wieder abgegebenen, betrugen 24,8si Millionen Mark, wovon 24,06 ` Millionen oder 96,7 Prozent für selbst abgeschlossene und 0,8 is Millionen oder 3,3 Prozent für in Rückdeckung übernommene Versicherungen zu rechnen sind. An Versicherungsleistungen wurden 9,823 Millionen Mark gewährt, nämlich 
9,385 Millionen für selbst abgeschlossene Versicherungen und 0,438 Millionen für Rückversicherungen, von den in der ausländischen Kapitalversicherung überhaupt fällig gewordenen Beträgèn kommen demnach 4,5 Prozent auf in Rückdeckung übernommene Versicherungen.

In der Rentenversicherung liefen Ende 1903 im Ausland 1116 Policen über 0,629 Millionen Mark Jahresrente, das sind 2,2 Prozent aller Policen und 3,6 Prozent der gesamten Jahresrenten. An Prämien wurden dafür vereinnahmt 0,422 Millionen Mark, wovon 0,420 Millionen auf selbst abgeschlossene Versicherungen kommen. Für Versicherungsleistungen waren 0,464 Millionen Mark bereit zu stellen, nämlich 0,435 Millionen für selbst abgeschlossene Versicherungen und 0,029 Millionen für in Rückdeckung übernommene Versicherungen; von den für ausländische Rentenversicherungen von den deutschen Gesellschaften überhaupt gezahlten Beträgen kommen also 6,3 Prozent auf in Rückdeckung übernommene Versicherungen.

Das aus-

landiscbe Geschäf der

deutschen und das deutsohe

Gegchsift der aus-

Isndlechen

Gesell-

schaften

$\mathrm{Zu}$ einer eingehenden Vergleichung des ausländischen Geschäfts der deutschen Unternehmungen mit dem deutschen Geschäfte der ausländischen Gesellschaften fehlt das Material, doch bieten folgende Zahlen wenigstens einige Anhaltspunkte:

Im Jahre 1903 hat die Prämieneinnahme betragen:

\begin{tabular}{|c|c|c|}
\hline insgesamt & $\begin{array}{l}\text { für selbst ab- } \\
\text { geschlossene } \\
\text { Versicherungen }\end{array}$ & $\begin{array}{c}\text { für in Rückdeckung } \\
\text { übernommene } \\
\text { Versicherungen }\end{array}$ \\
\hline $1000 \varkappa$ & 1000 U & 1000 d \\
\hline 25379 & 24472 & 907 \\
\hline 32699 & 32517 & 182 \\
\hline
\end{tabular}

bei den deutschen Gesellschaften aus dem ausländischen Geschäfte ............

bei den ausländischen Gesellschaften aus dem deutschen Geschäfte ............ 32517

182

Wenn hiernach auch die Prämieneinnahme für Rückversicherungen bei den deutschen Gesellschaften größer gewesen ist als bei den ausländischen Unternehmungen, so sind doch die Einnahmen für das unmittelbare Geschäft bei den deutschen Gesellschaften nicht unbeträchtlich hinter denen der ausländischen zurückgeblieben; insgesamt haben die deutschen Gesellschaften für Lebensversicherungen 7,320 Millionen Mark weniger an Prämien aus dem Auslande bezogen als die ausländischen Gesellschaften aus dem Deutschen Reiche eingenommen haben. Nicht minder groß sind die Unterschiede hinsichtlich der Zahlungen an Versicherungsleistungen, diese haben betragen:

\begin{tabular}{|c|c|c|c|}
\hline$\cdot$ & $\begin{array}{l}\text { insgesamt } \\
1000 \mathscr{M}\end{array}$ & $\begin{array}{c}\text { für selbst ab- } \\
\text { geschlossene } \\
\text { Versicherungen } \\
1000 \mathscr{M}\end{array}$ & $\begin{array}{c}\text { für in Rückdeckung } \\
\text { ubernommene } \\
\text { Versicherungen } \\
1000 \varkappa\end{array}$ \\
\hline $\begin{array}{l}\text { bei den deuschen Gesellschaften für ausländische } \\
\text { Versicherungen } \ldots \ldots \ldots \ldots \ldots \ldots \ldots \ldots \ldots \ldots \ldots \\
\text { bei den ansländischen Gesellschaften für deutsche }\end{array}$ & 10287 & 9820 & 467 \\
\hline Versicherungen . . . . . . . . . . & 17028 & 16899 & 129 \\
\hline
\end{tabular}

Die kleinen Pensionsund Sterbe kassen

Außer den besprochenen und in den Tabellen behandelten deutschen Versicherungsunternehmungen steht, wie bereits erwähnt, gemäß $\$ 2$ V.A.G. noch eine Anzahl kleiner Sterbe- und Pensionskassen unter Reichsaufsicht, die für eng begrenzte Kreise bestimmt und vielfach von gewerblichen Firmen nur für die eigenen Angestellten oder Arbeiter eingerichtet sind. Die Zahl dieser Gegenseitigkeitsvereine ist schwankend; einerseits sind diese Unternehmungen wohl kaum schon alle bekannt, auch entstehen immer neue, andererseits geht ihre Beaufsichtigung nicht selten gemäß $\S 3$ Abs. 2 nachträglich an die Landesbehörde über, manche lösen sich auf. Wie dem auch sei, die Zahl der unter Reichsaufsicht stehenden Sterbekassen und Pensionskassen wird immer nur sehr klein sein im Vergleiche zu der Zahl der unter Landesaufsicht befindlichen, und wenn hier einige Mitteilungen über jene gemacht werden, so können daraus keineswegs Schlüsse über die Bedeutung des Sterbekassen- und Pensionskassenwesens im Reiche gezogen werden. 
Für das Jahr 1903 haben insgesamt 62 solcher Sterbekassen dem Aufsichtsamte befriedigende Jahresberichte eingereicht. Danach ist die Zahl ihrer Mitglieder Ende r9oz auf rund 252000 und Ende r903 auf rund 258000 anzusetzen, ihre Einnahmen haben im Berichtsjahre 2897213 , $/$ und ihre Ausgaben $1746011 \mathscr{M}$. betragen.

Von den Einnahmen kommen 2369041 , $/$ oder 81,8 Prozent auf Beiträge für die Mitglieder, 12230 ، $/$ oder 0,4 Prozent auf Eintrittsgelder und 424097 ¿N/ oder 14,6 Prozent auf Vermögenserträge. Von den Ausgaben kommen $1241788 \mathscr{M}$ oder 71,1 Prozent auf Sterbegelder, 23810 A $M$ oder 1,4 Prozent auf Krankengelder, 25540 dt oder 1,5 Prozent auf Zahlungen für vorzeitig aufgelöste Versicherungen, 142053 ॥/l oder 8,1 Prozent auf Gewinnanteile und $175907 \mathscr{M}$. oder 10,1 Prozent auf Verwaltungskosten und Steuern. Das Vermögen dieser Kassen belief sich am Schlusse des Jahres r 903 auf $12318080 \mathrm{c} / \mathrm{l}$.

Die Zahl der Pensionskassen der genannten Art, die in befriedigender Weise über ihren Geschäftsbetrieb im Jahre 1903 an das Amt berichtet haben, betrug 57, sie hatten Ende 190243567 aktive Mitglieder, 1925 Pensionierte, 1097 Witwen und 404 Waisen und Ende 190345130 Aktive, 2192 Pensionierte, 1189 Witwen und 470 Waisen. Ihre Einnahmen betrugen im Berichtsjahre 5156208 , Nh und ihre Ausgaben 1834699 $/$. Von den Einnahmen kommen 3142789 eth oder 61,0 Prozent auf Beiträge für die Mitglieder, 122872 « oder 2,4 Prozent auf Eintrittsgelder und $1196744 \mathscr{M l}$ oder 23,2 Prozent auf Vermögenserträge. Von den Ausgaben kommen $1401150 \mathscr{H}$ oder 76,4 Prozent auf Pensionen, 38245 .ll oder 2,1 Prozent auf Krankengeld, 107762 , th oder 5,9 Prozent auf Sterbegeld und sonstige Leistungen an Versicherte, $75741 \mathscr{H}$ oder 4,1 Prozent auf Abfindungen beim Austritt aus der Kasse (meist infolge Wechsels der Dienststelle) und 104451 H oder 5,7 Prozent auf Verwaltungskosten und Steuern. Das Vermögen dieser Pensionskassen belief sich am Ende des Berichtsjahrs auf $32648820 \mathscr{M}$. Der niedrige Satz für Verwaltungskosten hat seinen Grund in erster Linie darin, daß bei den von gewerblichen Firmen eingerichteten Versicherungskassen die Verwaltungskosten meistens vollständig von den Firmen übernommen werden und daß bei andern Kassen die Verwaltung meist ehrenamtlich erfolgt.

\section{Gruppe II, Unfall- und Haftpflichtversicherung.}

Von den großen deutschen Versicherungsunternehmungen, welche der Reichsaufsicht Die Unterunterstehen, waren im Jahre 190330 auf dem Gebiete der Unfall- und Haftpflichtversicherung tätig, 29 Aktiengesellschaften und 1 Versicherungsverein auf Gegenseitigkeit. Von den Aktiengesellschaften ist eine, die Stuttgarter Mit- und Rückversicherungs-AktienGesellschaft, erst im Berichtsjahr unter Reichsaufsicht gekommen, weil sie den Geschäftsbetrieb in Zweigen aufgenommen hat, die der Reichsaufsicht unterstehen. Die Unfall- und Haftpflichtversicherung betrieb sie im Berichtsjahre nur im Wege der Rückversicherung. Da die Angaben, welche die Gesellschaft über diesen Teil ihres Geschäfts gemacht hat, nur sehr wenig eingehend sind, können sie hier nicht weiter als wie in Tab. II 3 behandelt werden.

Die übrigen Unternehmungen sind dieselben wie im Vorjahre. Von ihnen arbeiteten wie damals 24 sowohl in der Unfall- wie auch in der Haftpflichtversicherung; eine (Fides) betrieb von diesen beiden Zweigen nur die Haftpflichtversicherung und fünf (Friedrich Wilhelm, Teutonia, Magdeburger Lebens-Versicherungs-Gesellschaft, Nürnberger LebensVersicherungs-Bank und die Preußische National-Versicherungs-Gesellschaft) nur die Unfallversicherung.

Ebenso berichtet die vorliegende Statistik über dieselben sieben ausländischen $\mathrm{Ge}$ sellschaften wie die vorjährige. 
Versicherungsbestand der

Zur Beurteilung des Umfanges des Geschäfts der einzelnen Gesellschaften soll die Tab. II 1 dienen. Für die Gesamtheit der 29 Unternehmungen ist daraus zu entnehmen, daß am Ende des Jahres 1903 ohne die vom Allgemeinen Deutschen Versicherungs-Verein in Stuttgart in Rückdeckung genommenen Versicherungen in der Unfallversicherung 719669 und in der Haftpflichtversicherung 1246487 Policen über selbst abgeschlossene und in Rückdeckung übernommene Versicherungen in Kraft waren.

In der Haftpflichtversicherung wurden Versicherungen mit Gewinnbeteiligung der Versicherten von den Aktiengesellschaften überhaupt nicht abgeschlossen. Auch bei der Unfallversicherung kommen Versicherungen mit Gewinnbeteiligung nur wenig in Betracht. Von den Aktiengesellschaften haben nur fünf solche Versicherungen abgeschlossen, und darunter zwei auch nur in geringem Umfange. Von der Prämieneinnahme des Geschäftsjahrs in Höhe von $27917398 \mathscr{N}$. für selbst abgeschlossene Unfallversicherungen sämtlicher Unternehmungen kamen auf Versicherungen mit Gewinnbeteiligung nur 8147895 a d/ oder 29,2 Prozent, oder wenn man den Allgemeinen Deutschen Versicherungs-V'erein, weil er eine Gewinnbeteiligung nur auf Grund seines Charakters als Gegenseitigkeitsverein kennt, ausscheidet, 21,0 Prozent.

Was das Rückversicherungsgeschäft betrifft, so lassen sich dafür folgende Zahlen angeben. In der Unfallversicherung waren in Rückdeckung übernommen für $1346814 \mathfrak{N}$ Prämie oder 4,6 vom Hundert der ganzen Prämieneinnahme von $29264212 \mathscr{M}$ und in Rückdeckung gegeben für 4326421 l. oder für 14,8 vom Hundert der ganzen Prämieneinnahme. In der Haftpflichtversicherung waren in Rückdeckung übernommen für nur 1024263 l Prämie oder 3,8 vom Hundert und in Rückdeckung gegeben für $7615637 \mathfrak{M}$ oder 28,1 vom Hundert der gesamten Prämieneinnahme von $2 \tau 143211 \mathrm{~d}$.

In diesen Zahlen wie in allen folgenden sind bei dem Allgemeinen Deutschen Versicherungs-Verein in Stuttgart in der Haftpflichtversicherung auch die Versicherung gegen Wasserleitungsschäden und einige andere kleine Sachversicherungen mit enthalten.

Vergleich mit dem Vorjahre Ende des Vorjahrs (Tab. II 2), so findet man, daß die Zahl der Policen bei der Unfall-
versicherung um 39731 oder 5,8 Prozent und bei der Haftpflichtversicherung um
215386 oder 20,9 Prozent des Bestandes am Ende des Vorjahrs zugenommen hat. Die
gesamte Prämieneinnahme hat im Geschäftsjahre bei der Unfallversicherung 1111898 A
oder 3,9 Prozent und bei der Haftpflichtversicherung 4178608 ,ll oder 18,2 Prozent mehr
als im Vorjahre betragen. Ende des Vorjahrs (Tab. II 2), so findet man, daß die Zahl der Policen bei der UnfallEnde des Vorjahrs (Tab. II 2), so findet.man, daß die Zahl der Policen bei der Unfall-
versicherung um 39731 oder 5,8 Prozent und bei der Haftpflichtversicherung um
215386 oder 20,9 Prozent des Bestandes am Ende des Vorjahrs zugenommen hat. Die
gesamte Prämieneinnahme hat im Geschäftsjahre bei der Unfallversicherung $1111898 \mathscr{M}$
oder 3,9 Prozent und bei der Haftpflichtversicherung 4178608 .ll oder 18,2 Prozent mehr
als im Vorjahre betragen.

Wie schon erwähnt, betreiben sämtliche Gesellschaften außer der Unfall- und Haftbei pflichtversicherung auch noch andere Versicherungsgeschäfte, nur wenige von ihnen halten om aber in ihren Rechnungen die verschiedenen Versicherungszweige vollständig von einander getrennt, zumeist zerlegen sie nur gewisse für die verschiedenen $Z$ weige charakteristische Posten und führen andere als allen Zweigen gemeinsame Einnahmen und Ausgaben ungetrennt durch die Betriebsrechnung; zu diesen gehören namentlich der Gewinnvortrag und die Kapitalerträge einerseits und die Verwaltungskosten und Steuern sowie die Abschreibungen und die Verluste aus Kapitalanlagen andererseits, auch lassen sich die ..sonstigen" Einnahmen und Ausgaben vielfach nicht nach Versicherungszweigen scheiden. Das Aufsichtsamt strebt in seinen Vorschriften eine möglichst weitgehende Zerlegung auch dieser Posten an, es hat aber geglaubt, vorläufig mit seinen Anforderungen in dieser Hinsicht sich beschränken zu sollen; denn wenn auch bei manchen Gesellschaften für die Beurteilung des Umfanges, in welchem die einzelnen Versicherungszweige an den gemeinsamen Einnahmen und Ausgaben teilnehmen, ein zutreffender Maßstab ohne weiteres wird gefunden werden können, so sind doch die geschäftlichen Einrichtungen bei den Gesellschaften sehr verschieden; es wird bei manchen Unternehmungen einstweilen schwer sein, 
die gemeinsamen Posten in einwandfreier Weise zu zerlegen. Das Amt hat deshalb, wenigstens für die ersten Jahre, davon abgesehen, für jeden Versicherungszweig, den eine solche Gesellschaft pflegt, eine vollständige Betriebsrechnung zu verlangen oder selbst aufzustellen. Um aber bei einheitlichem Vorgehen nach Möglichkeit erkennen zu lassen, wie weit die einzelnen Versicherungszweige an dem Gesamtgeschäfte beteiligt sind, ist folgendermaßen verfahren. Es sind für jeden Zweig, in dem eine Gesellschaft arbeitet, diejenigen Einnahme- und Ausgabeposten des G'eschäftsjahrs, die bei allen Unternehmungen mit Sicherheit als dem betreffenden Zweige allein zugehörig bestimmt werden können, als dessen S onderEinnahmen und Sonder-Ausgaben zusammengestellt, und zwar stets bei der Gruppe, zu welcher der Zweig gehört. Demnach sind in jeder Gruppe die Sonder-Einnahmen und Sonder-Ausgaben aller derjenigen Gesellschaften zusammengestellt, welche auf einem zu der fraglichen Gruppe gehörigen Versicherungsgebiete tätig sind. Die Gewinn- und Verlustrechnung beginnt dann mit der "Summe der Sonder-Einnahmen aller Zweige" einerseits und der "Summe der Sonder-Ausgaben aller Zweige" andererseits, und sie ist dann, wenn eine Gesellschaft für jeden Zweig eine besondere Rechnung aufstellt, bei der Gruppe, zu welcher der Zweig gehört, anderenfalls bei derjenigen, zu welcher die Gesellschaft nach ihrem Hauptgeschäfte - die Transportversicherung ausgeschlossen - $\mathrm{zu}$ rechnen ist, gegeben. Sofern eine Gesellschaft nur einen Zweig bearbeitet oder für einen Zweig eine vollständig durchgeführte Betriebsrechnung geliefert hat, reduziert sich die Summe der Sonder-Einnahmen und Ausgaben aller Zweige auf die Summe der Sonder-Einnahmen und Ausgaben eben dieses Zweiges.

Als Sonder-Einnahmen sind bei Gruppe II verrechnet die Prämien für das Geschäftsjahr, d. h. die Prämien unler Berücksichtigung der C̈berträge aus dem Vorjahr und der Überträge auf das folgende Jahr, einschließlich der an die Rückversicherungsgesellschaften etwa gezahlten Rückversicherungsprämien, ferner die Policegebühren, die Vergütungen der Rückversicherer, der Unterschied zwischen der Schadenreserve aus dem Vorjahr und den Zahlungen und Rückstellungen für Versicherungsfälle der Vorjahre aus selbst abgeschlossenen Versicherungen, sofern die Schadenreserve größer ist, und der etwaige Mehrbetrag der aus dem Vorjahr übernommenen gegenüber der am Schlusse des Jahres zu stellenden Prämienreserve. Die Sonder-Ausgaben setzen sich bei Gruppe II zusammen aus den Zahlungen für selbst abgeschlossene und für in Rückdeckung übernommene Versicherungen einschließlich des Anteils der Rückversicherer, aus den Rückversicherungsprämien, dem Unterschiede zwischen der Schadenreserve aus dem Vorjahr und den Zahlungen und Rückstellungen für Versicherungsfälle der Vorjahre aus selbst abgeschlossenen Versicherungen, sofern die Schadenreserve kleiner ist, und endlich aus der Zunahme der Prämienreserve. Es soll hier aber besonders betont werden, da $\beta$ der Unterschied zwischen der Schaden reserve aus dem Vorjahr und den Zahlungen und Rückstellungen für Versicherungsfälle. der Vorjahre allein keinen Schluß darüber zuläßt, ob die Schadenreserve zulänglich bemessen war. Denn einerseits sind aus der Schadenreserve auch andere Leistungen als Zahlungen für Versicherungsfälle der Vorjahre zu bewirken, z. B. Zahlungen für in Rückdeckung übernommene Versicherungen und Zuführungen zur Prämienreserve, und andererseits wird die Schadenreserve meist ohne den Anteil der Rückversicherer eingestellt, wogegen die Zahlungen einschließlich dieses Anteils erscheinen.

Die Sonder-Einnahmen haben, wenn man von der Stuttgarter Mit- und Rückver- DieSondersicherungs-Aktien-Gesellschaft absieht, insgesamt bei den 29 deutschen Gesellschaften und Aus$67562709 \mathscr{M}$ betragen, davon kamen auf Prämien für Unfallversicherung 28893034 Nl. gaben des oder 42,8 vom Hundert, auf Prämien für Haftpflichtversicherung $26036007 \mathscr{H}$ oder ${ }_{\text {jabrs }}^{\text {Besichts: }}$ 38,5 vom Hundert und auf Vergütungen der Rückversicherer $10939547 \mathscr{M}$ oder 16,2 vom Hundert aller Sonder-Einnahmen. 
Die Summe der Sonder-Ausgaben beziffert sich auf 44801314 , d, davon entfallen $22374156 \mathscr{M}$ auf Zahlungen für Versicherungsfälle im Geschäftsjahr aus selbst abgeschlossenen Versicherungen, und zwar (Tab. II 4) 13329057 \% oder 29,8 Prozent aller Sonder-Ausgaben auf Unfallversicherungsfälle, ferner $7137391 \mathrm{Nt}$ oder 15,9 Prozent auf Haftpflichtversicherungsfälle, $1120910 \mathscr{M}$ oder 2,5 Prozent auf laufende Renten und endlich 786798 alt oder 1,8 Prozent auf Prämienrückgewährbeträge. Von der Summe der Sonder-Ausgaben kommen ferner 2962540 At oder 6,6 Prozent auf Vergütungen für in Rückdeckung übernommene Versicherungen (darunter 1791223 ॥ oder 4,0 Prozent für eingetretene Unfallversicherungsfälle und $543773 \mathscr{M}$ oder 1,2 Prozent für eingetretene Haftpflichtversicherungsfälle), 4325184 etl oder 9,7 Prozent für Rückversicherungsprämien für Unfallversicherungen und 7642638 , th oder 17,1 Prozent für Rückversicherungsprämien für Haftpflichtversicherungen.

Schadenzahlungen

$$
\text { (54 }
$$
Schadenzahlungen verausgabt wurden, weitere $84 \mathscr{N}$. wurden zur Erhöhung der Prämienreserve verwandt. Tatsächlich ist allerdings für eigentliche Schäden noch etwas weniger aufgewandt worden; denn einzelne Gesellschaften verrechnen bei den Schadenzahlen auch noch mancherlei andere Beträge, z. B. Regulierungskosten.

Die Gewinn- und Verlustrechnung selbst (Tab. II 6) stellt das Ergebnis des Geschäftsjahrs dar; der Gewinnvortrag aus dem Vorjahr ist fortgelassen, auch sind hier Reserven und Rücklagen, wie schon bei der Ermittelung der Sonder-Einnahmen und Sonder-Ausgaben nicht mit ihrem ganzen Betrage zu Anfang des Jahres in Einnahme und am Schlusse des Jahres in Ausgabe nachgewiesen, vielmehr nur ihre Änderungen, die der Betrieb des Berichtsjahrs zur Folge hatte, verrechnet.

Von den 29 Gesellschaften scheiden für die Gewinn- und Verlustrechnung hier 7 aus, w.eil sie ihrem Hauptgeschäfte nach in eine andere Gruppe gehören und für die Unfallund Haftpflichtversicherung keine vollständige Betriebsrechnung aufgestellt haben. Die für sie in Tab. II 3 gegebenen Sonder-Einnahmen und Ausgaben aus der Unfall- und Haftpflichtversicherung sind da weiter verrechnet, wo sich die Gewinn- und Verlustrechnung dieser Gesellschaften befindet, nämlich für die Providentia bei Gruppe I und bei den anderen (Fides, Thuringia, Neptun, Transatlantische Feuer-Versicherungs-Gesellschaft, Rheinland und Preußische National-Versicherungs-Gesellschaft) bei Gruppe IV.

Die übrigen 22 Gesellschaften weisen in der Gewinn- und Verlustrechnung (Tab. II 6) als Summe der Sonder-Einnahmen aller Zweige den Betrag von $109214538 \mathscr{M}$ auf und als Summe der Sonder-Ausgaben aller Zweige den Betrag von $81138738 \mathscr{M}$, davon stammen aus der Unfall- bezw. Haftpflichtversicherung allein 63244031 dh und $41928685 \mathscr{A l}$ oder 57,9 und 51,7 Prozent. Den Hauptanteil hat daneben die Transportversicherung, die bei der Allianz, der Albingia, der Norddeutschen Versicherungs-Gesellschaft, der Mannheimer und der Oberrheinischen Versicherungs-Gesellschaft die Unfall- und Haftpflichtversicherung an Bedeutung beträchtlich überragt (Tab. II 5).

Die gesamte Jahreseinnahme aller 22 Gesellschaften, soweit sie bei Gruppe II zu verrechnen ist, stellt sich auf $114689941 \mathscr{M}$, die gesamte Jahresausgabe auf $105620096 \mathscr{M}$. Eine Gesellschaft, die hier nur mit dem Unfallversicherungsgeschäfte vertreten ist, hat aus diesen Betrieben während des Jahres 1903 einen Verlust von zusammen $43756 \mathfrak{M}$ gehabt, die übrigen einen Gewinn von insgesamt 9113601 ،/l, wieviel hiervon auf die einzelnen Zweige kommt, läßt sich nicht ermitteln. 
Von den gesamten Einnahmen entfallen auf die Sonder-Einnahmen $109214538 \mathcal{M l}$ oder 95,2 Prozent, auf den Ertrag der Kapitalanlagen 4717925 \& oder 4,1 Prozent, von den gesamten Ausgaben auf die Sonder-Ausgaben 81138738 dl oder 76,8 Prozent, auf Verwaltungskosten und Steuern $22957531 \mathcal{M}$ oder 21,7 Prozent und auf Abschreibungen 253335 d oder 0,3 Prozent.

Die Gewinn- und Verlustrechnung, wie sie in Tab. II 6 gegeben ist, umfaßt außer dem eigentlichen Versicherungsgeschäft auch das Ergebnis der Vermögensverwaltung der Gesellschaften. Über das Ergebnis des Versicherungsbetriebs allein erhält man ein annähernd zutreffendes Bild, wenn man die Summe der Sonder-Einnahmen der Summe der Sonder-Ausgaben und den Verwaltungskosten nebst Steuern gegenüberstellt. Dann findet man, da 3 außer der schon erwähnten Gesellschaft noch zwei andere einen Fehlbetrag aufzuweisen haben, oder daß diese Gesellschaften den Gewinn, den ihre Gewinnund Verlustrechnung ausweist, lediglich den Ergebnissen ihrer Vermögensverwaltung verdanken. Nach dieser Rechnung haben 19 Gesellschaften aus dem Versicherungsbetrieb im Geschäftsjahr einen Gewinn von insgesamt 5221316 ، $/$ und die übrigen drei einen Verlust von 103047 .// erzielt. Es ist danach das Berichtsjahr etwas günstiger verlaufen als das Vorjahr, indem von denselben Gesellschaften 17 einen Gewinn von zusammen 4657053 ، $/$ erzielt und fünf einen Verlust von 252828 ، $/$ erlitten haben.

Eine Bilanz ist in dieser Statistik grundsätzlich nur für das Gesamtgeschäft einer Die Bllanz jeden Gesellschaft gegeben und bei derjenigen Gruppe aufgeführt, zu der das Unternehmen seinem Hauptbetriebe nach - Transportversicherung immer ausgeschlossen gehört. So bleiben bei Gruppe II von den 29 deutschen Gesellschaften, welche Unfall- oder Haftpflichtversicherungen direkt abschließen, nur 11 übrig. Von den anderen findet sich die Bilanz von Friedrich Wilhelm, Victoria, Urania, Vaterländische Lebens-VersicherungsGesellschaft, Providentia, Janus, Teutonia, Atlas, Magdeburger Lebens-VersicherungsGesellschaft, Wilhelma, Bayerische Hypotheken- und Wechsel-Bank und Nürnberger Lebens-Versicherungs-Bank bei Gruppe I (Tab. I 42), und die Bilanz von Fides, Thuringia, Neptun, Transatlantische Feuer-Versicherungs-Gesellschaft, Rheinland und Preußische National-Versicherungs-Gesellschaft bei Gruppe IV (Tab. IV 24).

Aus den in Tab. II 7 für die 11 Gesellschaften gegebenen Bilanzen mögen hier die folgenden Schlußzahlen Platz finden:
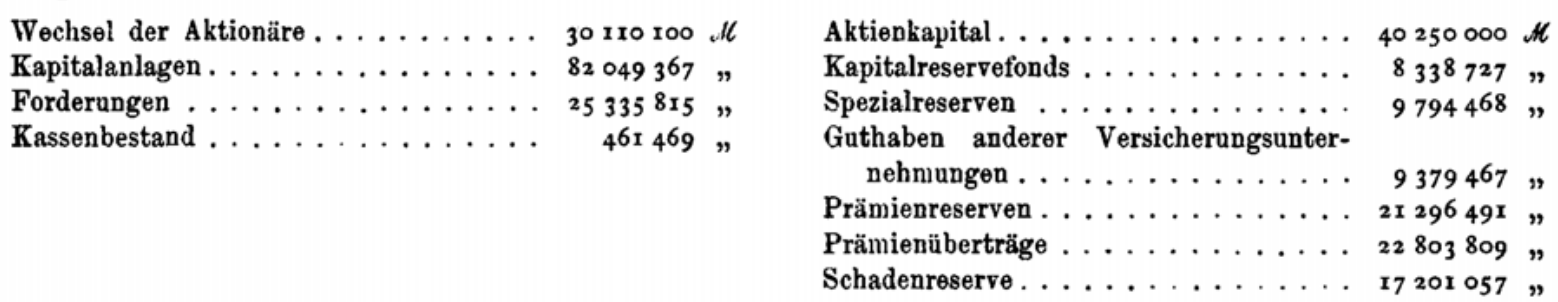

Dabei ist zu erwähnen, daß aus den Nebenbetrieben der Gesellschaften von den Prämienreserven 14169785 dl auf Lebensversicherungen und von den Prämienüberträgen $8190018 \%$ auf nicht zur Gruppe II gehörige Versicherungszweige kommen; für die Unfall- und Haftpflichtversicherung bleiben danach an Prämienreserven nur 7126706 , 1 . und an Überträgen $14613791 \mathrm{c}$.

Die Bilanz für Ende 1903 schließt für 11 Gesellschaften mit 138752830 NH ab, davon kommen bei den Aktiven 21,7 Prozent auf Wechsel der Aktionäre, 59,1 Prozent auf Kapitalanlagen, 18,3 Prozent auf Forderungen, 0,3 Prozent auf den Kassenbestand und 0,6 Prozent auf andere Aktiva; bei den Passiven 29,0 Prozent auf Aktienkapital, 6,0 Prozent auf Kapitalreserve, 7,1 Prozent auf Spezialreserve, 6,8 Prozent auf Guthaben bei anderen Versicherungsunternehmungen, 1อ̆,3 Prozent auf Prämienreserve, 16,4 Prozent auf Prämienüberträge, 12,4 Prozent auf Schadenreserve und 7,0 Prozent auf andere Passiva. 
Die Kapitalanlagen (82049367 $\mathcal{N}$ ) verteilen sich auf folgende Wertarten: 7175507 , oder 8,7 Prozent Grundbesitz, 48502363 , 1l oder 59,1 Prozent Hypotheken, 25412872 all. oder 31,0 Prozent Wertpapiere, 224000 , /l oder 0,3 Prozent Darlehen auf Wertpapiere, 426528 , 1 . oder 0,5 Prozent Policedarlehen und 308097 , 1 oder 0,+ Prozent Wechsel. Im Vergleich mit dem Vorjahre hat zugenommen der Grundbesitz um 1402874 \&ll, der Besitz an Hypotheken um $4843064 \mathscr{N}$, an Wertpapieren um $3701309 \mathscr{N}$, die Policedarlehen sind um '156080 $\mathrm{N}$ und die Darlehen auf Wertpapiere um $224000 \mathrm{Ml}$. gewachsen. Dagegen hat der Besitz an Wechseln um 200669 , $/$ abgenommen. Sieht man von den Darlehen auf Wertpapiere und von den Policedarlehen, die den öffentlichen Geldverkehr nicht direkt berühren, ab, so haben die deutschen Unfall- und Haftpflichtversicherungsgesellschaften auf dem Geldmarkte für netto 9 วั45 909 № Anlagen erworben.

Die Forderungen (25335815 $\mathrm{NC}$ ) bestehen aus: 6070378 $/$ oder 24,0 Prozent Guthaben bei Banken, 3730365 oder 14,7 Prozent Guthaben bei anderen Versicherungsunternehmungen, 713279 Al oder 2,8 Prozent rückständigen Zinsen und Mieten, 12374278 , oder 48,8 Prozent Ausständen bei Agenten und 2447515 Al oder 9,7 Prozent gestundeten Prämien.

Jede der 11 Gesellschaften hat mit einem Gewinn abgeschlossen, der sich für alle zusammen nach der Bilanz auf $5899686 \mathscr{N}$. oder mit Einschluß des Gewinnvortrags aus dem Vorjahr auf 6137382 Ml stellt gegen 5143807 und 5358028 M im Vorjahre. Von dem Gewinne des Berichtsjahrs kommen auf die Lebensversicherungsabteilung des Allgemeinen Deutschen Versicherungs-Vereins in Stuttgart 311605 Nh, dessen Verteilung aus Tabelle I 45 zu ersehen ist. Von dem Reste, $5825775 \mathscr{M}$, sind nach Tabelle II 9 überwiesen $98569 \mathscr{M}$ $(1,7$ Prozent) an den Kapitalreservefonds, $679558 \mathcal{H}$ (11,7 Prozent) an sonstige Reserven, $150400 \mathscr{N}(2,6$ Prozent) an die Beamtenfonds, $1944067 \mathscr{N}$ (33,4 Prozent) an die Aktionäre. Ferner sind davon ausgegeben $559580 \mathscr{M}$ (9,6 Prozent) an Tantiemen, $1879658 \mathscr{M}$ (32,3 Prozent) Gewinnanteile an die Versicherten sowie $112757 \mathscr{M}$ (1,9 Prozent) anderweit, endlich sind $401186 \mathscr{M}$ (6,9 Prozent) auf neue Rechnung vorgetragen.

Die Kapitalreserven stehen in der diesjährigen Bilanz um $192913 \mathscr{M}$, und die Spezialreserven um $1300308 \mathscr{N}$. höher als in der Bilanz für Ende rgoz (Tab. II 11).

Das aus-

ländische

Geschät

der

deutschen

nehmungen

Von den hier behandelten 29 deutschen Unternehmungen haben 16 auch mehr oder weniger umfangreiche Unfall- und Haftpflichtversicherungsgeschäfte im $\boldsymbol{x}$ Auslande gemacht, nämlich in Bulgarien, Finnland, Griechenland, Japan, Portugal und Rumänien je 1, in England, Rußland, Spanien, der Türkei und in den Vereinigten Staaten von Amerika je 2, in Italien, Luxemburg und Ungarn je 3, in Frankreich und Norwegen je 4, in Schweden 5, in der Schweiz 8, in Belgien 9, in Österreich 10, in Dänemark 11 und endlich in den Niederlanden 13.

Sie hatten im Auslande 50113 Versicherungen laufen, das sind 2,5 Prozent aller Versicherungen. Die vereinnahmte Prämie, einschließlich der davon für Rückversicherungen wieder abgegebenen, betrug für das ausländische Geschäft insgesamt 6,532 Millionen Mark, wovon 5,798 Millionen Mark für selbst abgeschlossene und 0,742 Millionen Mark für in Rückdeckung übernommene Versicherungen zu rechnen sind, im Verhältnisse zum Gesamtgeschäfte sind das 11,6 Prozent, 10,7 Prozent und 31,3 Prozent. Beim ausländischen Geschäfte machten die Prämien für das in Rückdeckung übernommene Geschäft 11,4 Prozent von denen für das ganze ausländische Geschäft aus. Für Versicherungsleistungen wurden 3,982 Millionen Mark gewährt, nämlich 3,523 Millionen Mark für selbst abgeschlossene Versicherungen und 0,459 Millionen Mark für Rückversicherungen, von der Gesamt-Ausgabe für ausländische Schäden kommen demnach 11,5 Prozent auf in Rückdeckung übernommene Versicherungen. Auf $1000 \mathrm{At}$. Prämieneinnahme kommen $610 \mathrm{~N}$ Schadenzahlungen. Die Versicherungsleistungen entsprechen zwar nicht genau aber doch angenähert dem, was für das Gesamtgeschäft oben als Sonder-Ausgaben bezeichnet worden 
ist, wenn man die Rückversicherungsprämien davon absetzt, sodaß man sie wohl dazu in Beziehung setzen darf, sie betragen dann 12,1 Prozent davon.

Außer diesen großen Unternehmungen unterlagen im Jahre 1903 der Reichsaufsicht kleine noch einige kleine Versicherungsvereine auf Gegenseitigkeit, von denen hier der Kirch- sicherungs- $_{\text {ver- }}$ liche Ver'sicherungsverein auf Gegenseitigkeit in Cöln a. Rh., der Imkerversicherungs- vereine verein für die Provinz Hannover und angrenzende Gebiete in Hannover mit seiner Haftpflichtversicherungsabteilung und der Haftpflichtversicherungsverein Sommersdorf und Umgebung in Sommersdorf $\mathrm{zu}$ nennen sind, und drei eingetragene Genossenschaften mit beschränkter Haftpflicht, das sind von Mitgliedern der Berufsgenossenschaften gegründete Verbände zur Versicherung gegen Unfall und Haftpflicht, nämlich die Unfall-Genossenschaft der Deutschen Steinindustrie, die Unfall-Genossenschaft der Deutschen Tiefbauunternehmer und die Unfall-Haftpflicht-Genossenschaft der Besitzer von Ziegeleien und verwardten Betrieben, sämtlich mit dem Sitze in Berlin.

Die Zahl der Policen dieser Unternehmungen belief sich Ende 1903 auf 5002 . Die Prämien für das Geschäftsjahr betrugen für alle Unternehmungen zusammen 76580 «t. Davon wurden für Rückversicherungen 4876 dl wieder abgeführt. Für Schäden wurden 30679 . /, für Verwaltungskosten 10075 , /l verausgabt. Das Geschäftsjahr ist für alle Vereine günstig verlaufen, es hat ihnen einen Gewinn von insgesamt 44066 ، $/$ (einschließlich 246 ، / Gewinnvortrag aus dem Vorjahre) gebracht. Davon sind 14183 $/$ den Kapitalreservefonds, 16011 , /l den sonstigen Reserven und 1447 , $/$ l den Garanten überwiesen worden; $4500 \mathrm{dl}$ sind an Tantiemen ausgegeben, $6549 \mathrm{Al}$ den Versicherten gutgebracht und 1376 , th auf neue Rechnung vorgetragen worden.

Die Bilanz für alle diese Unternehmungen schließt mit 496393 ، /. ab. Als Hauptposten der Aktiva sind zu vermerken 297406 ./ Kapitalanlagen, 102384 d Forderungen (darunter 76657 , $/$ Guthaben des Kirchlichen Versicherungsvereins beim Erzbischöflichen Stuhle zu Cöln) und 8919 \% Kassenbestand, als Hauptposten der Passiva 196619 «. Garantiekapital, 182719 , $H$. Kapitalreservefonds und $57187 \mathscr{M}$. Schadenreserve.

Zur Erläuterung des Umfanges des deutschen Geschäfts der ausländischen Unternehmingen in der Unfall- und Haftpflichtversicherung sollen die Tab. II 12 bis 14 dienen

Fie Basler Lebensversicherungsgesellschaft hat nur in der Únfallversicherung GeGeschäft ausländischer schäfte gemacht. Von den anderen Gesellschaften betrieben im Deutschen Reiche neben schaften der Unfall- und Haftpflichtversicherung die Zürich auch die Kautionsversicherung, die Schwezerische National-Versicherungs-Gesellschaft auch die Einbruchdiebstahl-, die Glas- und die Transportversicherung, die Schweizerische Unfallversicherungs-Aktiengesellschaft in Winterthur die Einbruchdiebstahl- und die Kautions- und Garantieversicherung.

Sieben ausländische Gesellschaften haben aus ihrem Geschäft im Deutschen Reiche für $19^{\circ} 36425054$ ، /l Prämien für selbst abgeschlossene Unfallversicherungen, 27316 ॥. Prämiın für in Rückdeckung übernommene Versicherungen eingenommen und davon 994316 dl für Rückversicherung wieder ausgegeben.

An dem gesamten Geschäfte der Gesellschaften in der Unfallversicherung war, was die Prämieneinnahme betrifft, das deutsche Geschäft mit 21,1 Prozent, und was die Ausgabe für Rückversicherungsprämien betrifft, mit 29,1 Prozent beteiligt.

I 1 der Haftpflichtversicherung haben sechs ausländische Gesellschaften aus dem deutscien Geschäfte 4508872 ، $/$ eingenommen, davon 26299 ، oder 0,6 Prozent für in Rückdeckung übernommene Versicherungen, diese Prämieneinnahme betrug 22,0 Prozent ihres gesamten Haftpflichtversicherungsgeschäfts. Für Rückversicherungen wurden von dem ceutschen Geschäfte 936137 // oder 20,8 Prozent der Prämien wieder ausgegeben, von dem Gesamtgeschäfte 21,t Prozent. 
Für selbst abgeschlossene Versicherungen hatten die Gesellschaften folgende Beträge bereitzustellen: $3650009 \mathscr{M}$. für Unfallversicherungsfälle, $1298906 \mathscr{M}$ für Haftpflichtversicherungsfälle, $238159 \mathscr{M}$ für laufende Renten und 14318 cll für Prämienrückgewährbeträge, zusammen 5201392 N. Für Versicherungsfälle aus in Rückdeckung übernommenen Versicherungen handelte es sich um 30462 . für eingetretene Unfallversicherungsfälle, 97352 N für Haftpflichtversicherungsfälle und 9880 Nh für vorzeitige Auflösungen, zusammen $137694 \mathcal{H}$. Das Geschäftsjahr 1903 hat diesen Gesellschaften eine Ausgabe von insgesamt 5339086 N für Schadenfälle und für Renten verursacht, in welchem Umfange hieran die Rückversicherer beteiligt sind, kann nicht angegeben werden. Diesen Schäden steht eine Bruttoprämieneinnahme - allerdings ohne Berücksichtigung der Überträge - von $10961242 \mathscr{N}$. gegenüber, von $1000 \mathscr{N}$. Prämien sind demnach durchschnittlich $487 \mathscr{N}$. für Schäden ausgegeben.

Bei denjenigen Gesellschaften, bei denen nach Tab. II 13 ein Vergleich des Berichtsjahrs mit dem Vorjahr angängig ist, ist bei der Unfallversicherung die Zahl der Policen von 73666 auf 78114 oder um 6,0 Prozent gestiegen, die Prämieneinnahme von $6009372 \mathscr{N}$ auf 6252828 N oder um 4,1 Prozent; bei der Haftpflichtversicherung die Zahl der Policen von 99036 auf 122848 oder um 24,0 Prozent, die Prämieneinnahme von 3807155 \% auf $4404176 \mathscr{M}$ oder um 15,7 Prozent.

Der Jahres abschluB der aus-

ländischen achaften

Zur Beurteilung der ganzen Geschäftsgebarung und des Vermögensstandes der ausländischen Gesellschaften sind in diese Statistik auch die Gewinn- und Verlustrechnungen und die Bilanzen der Gesellschaften aufgenommen, wie sie für das ganze Geschäft der Gesellschaften (nicht bloß für das deutsche) gelten. Dabei ist möglichst nach denselben Grundsätzen und in denselben Formen vorgegangen wie bei den deutschen Unternehmungen, es wird daher besonderer Erklärungen nicht bedürfen.

Die Gewinn- und Verlustrechínung (Tab. II 16) ist für sechs Gesellschaften zu geben; für eine, den Österreichischen Phönix, ist sie nach den hier befolgten Grundsätzen bei Gruppe IV nachzuweisen. Die Rechnung der Schweizerischen National-VersicherungsGesellschaft umfaßt auch das Geschäft in der Transport-, der Einbruchdiebstahl- und der Glasversicherung.

Die Bilanz ist hier nur für fünf Gesellschaften zu geben (Tab. II 17), indem die für den Österreichischen Phönix nach Gruppe IV und die für die Basler Lebens-Versicherungs-Gesellschaft nach Gruppe I genommen ist.

Hinsichtlich der Kapitalanlagen dieser fünf Gesellschaften ist zu bemerken, daß die beiden österreichischen Unternehmungen von ihrem ganzen Bestande von $15989390 \mathcal{A l}$. in Grundbesitz $1061197 \mathscr{N}$. oder 6,6 Prozent und den Rest $14928193 \mathscr{M l}$. oder 93,4 Prozent in Wertpapieren angelegt hatten, und daß von dem gesamten Bestande (33093597 , /l) der drei schweizerischen Gesellschaften 3352000 d oder 10,1 Prozent in Grundbesitz, 10464560 Al. oder 31,6 Prozent in Hypotheken und 19277037 , N. oder 58,3 Prozent in Wertpapieren angelegt waren.

Das ausländische Gesobaft der deutschen und das deutsche Geschäft der ausländisohen Gesellschaften

Ein eingehender Vergleich des ausländischen Geschäfts der deutschen Unternehmungen mit dem deutschen Geschäfte der ausländischen Unternehmungen auf dem Gebiete der Unfall- und Haftpflichtversicherung kann zwar nicht gegeben werden, indessen verdienen doch folgende Zahlen vorgeführt zu werden.

Im Jahre 1903 hat betragen die Bruttoprämieneinnahme

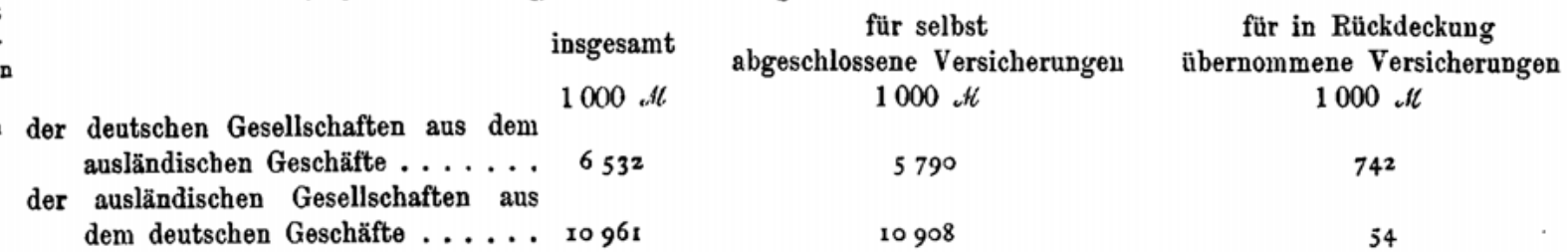


Während also die Einnahmen an Rückversicherungsprämien bei den deutschen Gesellschaften größer gewesen sind als bei den ausländischen Unternehmungen, bleiben die für das unnittelbare Geschäft bei den deutschen Gesellschaften hinter denen der ausländischen beträchtlich zurück; insgesamt haben die deutschen Gesellschaften für Unfall- und Haftpflichtversicherungen 4429686 . $/$ weniger an Prämien aus dem Auslande bezogen als die ausländischen Gesellschaften aus dem Deutschen Reiche eingenommen haben.

Für Versicherungsleistungen sind gezahlt worden

\begin{tabular}{|c|c|c|c|}
\hline & insgesamt & $\begin{array}{c}\text { für selbst } \\
\text { abgeschlossene Versicherungen }\end{array}$ & $\begin{array}{l}\text { für in Rückdeckung } \\
\text { übernommene Versicherungen }\end{array}$ \\
\hline n den deutschen Gesellschaften für & 1000 dt & 1000 d & 1000 d \\
\hline $\begin{array}{l}\text { ausländische Versicherungen } \ldots . . . \\
\text { on den ausländischen Gesellschaften }\end{array}$ & 3982 & 3523 & 459 \\
\hline für deutsche Versicherungen $\ldots \ldots$ & 5339 & $520 x$ & r 38 \\
\hline
\end{tabular}

\section{Gruppe III, Landwirtschaftliche Versicherung.}

Für diese Gruppe kommen zur Zeit nur die Viehversicherung und die Hagelversicherung in Frage, sonstige landwirtschaftliche Versicherungen unterstanden im Jahre 1903 der Reichsaufsicht nicht.

\section{Wiehversicherung.}

Die Viehversicherung wird im Deutschen Reiche außer von einigen größeren Unter- Die Unternehmungen von mehreren tausend kleinen Vereinen von lokaler Bedeutung betrieben. Davon unterliegt der Reichsaufsicht nur ein kleiner Bruchteil, und dieser Bruchteil enthält wieder eine Anzahl Vereine von so untergeordneter Bedeutung, daß sich ihre statistische Betrachtung nur in Verbindung mit der Gesamtheit aller kleinen Unternehmungen lohnen wird, die späteren Jahren vorbehalten bleibt.

Die vorliegende Statistik beschränkt sich daher auf eine kleine Zahl größerer Gegenseitigkeitsvereine. nämlich auf die in der vorjährigen Statistik behandelten 20 Vereine mit Ausnahme der Bremer Vieh-Versicherungs-Gesellschaft auf Gegenseitigkeit, die wegen Unzulänglichkeit ihres Berichts ausfallen mußte, und auf fünf Vereine, die im vorigen Jahre aus demselben Grunde nicht mit bearbeitet werden konnten, das sind: die Altenburger Vieh-Versicherungs-Gesellschaft auf Gegenseitigkeit zu Altenburg, zùm Geschäftsbetriebe zugelassen im Jahre 1903 - sie ist aus der 1888 gegründete Altenburger ViehVersicherungs -Gesellschaft, Eingetragene Genossenschaft mit beschränkter Haftpflicht, hervorgegangen -; die Braunschweigische Allgemeine Vieh-Versicherungs-Gesellschaft auf Gegenseitigkeit zu Braunschweig, gegründet 1888 ; die Lenzener Viehversicherungs-Gesellschaft auf Gegenseitigkeit zu Lenzen (Elbe), gegründet r889; die Pferde-Versicherung auf Gegenseitigkeit Sommersdorf und Umgegend in Sommersdorf, gegründet 1896 , und der Viehversicherungs-Verein des Rhein- und Maingaus zu Wiesbaden, gegründet 1877 . Ferner sind in die vorliegende Statistik aufgenommen die Hamburg-Altonaer Viehversicherung (G. m.b.H.) zu Altona, die Schlachtviehversicherung von Heinrich Koch in Bernburg, die National-Versicherung gegen Trichinengefahr in Cassel, die Anhaltische Trichinen-Versicherungs-Anstalt C. Irmer in Köthen, die Ostrauer Schlachtvieh-Versicherung von G. Boerner \& Uhlmann, Inhaber August Uhlmann in Halle, die Hamburger Schlachtvieh-Versicherung Gebr. Skriver in Hamburg und endlich die Allgemeine deutsche Schlachtvieh-Yersicherungsanstalt Jauer, Friedrich Bohne in Jauer. 
Der Verein Halensia betreibt auch die Glasversicherung, doch ist dieses Geschäft so klein, daß es nicht erforderlich erschien, in der Gewinn- und Verlustrechnung die Glasversicherung auszuscheiden. Alle anderen Unternehmungen befassen sich nur mit der Viehversicherung.

Die Gegenseitigkeitsvereine betreiben sämtlich die V'iehlebensversicherung, sechs von ihnen auch die Schlachtviehversicherung, zwölf noch andere Nebenarten der Viehversicherung und eine, die Altenburger Vieh-Versicherungs-Gesellschaft, außer der Viehversicherung noch die Sachschäden-Haftpflicht-Versicherung. Die übrigen Unternehmungen schließen Viehlebensversicherungen überhaupt nicht ab, sie wirken sämtlich und ausschließlich auf dem Gebiete der Schlachtviehversicherung, nur die Hamburger Schlachtviehversicherung Gebr. Skriver betreibt auch die Quarantäneversicherung.

Aktiengesellschaften, welche auf dem Gebiete der Viehversicherung arbeiten, waren nicht vorhanden.

Ausländische Unternehmungen waren in der Viehversicherung zum Geschäftsbetrieb im Deutschen Reiche nicht befugt.

Umfang der Versicbernng, Prämien, Scbäden

Der gesamte Umfang des Geschäfts der hier zu behandelnden Unternehmungen läßt sich nicht genau feststellen, weil bei zwei Gegenseitigkeitsvereinen nur der Betrag, der am Ende des Jahres versichert war, bekannt geworden ist, und weil ein anderes Unternehmen, die Hamburg-Altonaer-Viehversicherungs-Gesellschaft, überhaupt keine Angaben über die Versicherungssummen gemacht hat. Man hat sich daher, auch wenn man sich auf die 24 Gegenseitigkeitsvereine und die nach Ausscheiden der genannten Gesellschaft verbleibenden 6 sonstigen Unternehmungen beschränkt, gegenwärtig $\mathrm{zu}$ halten, daß die folgenden Zahlen etwas zu klein sind.

Nach Tab. III 1 hatten die sämtlichen in Betracht kommenden 30 Unternehmungen im Berichtsjahre Versicherungen im Betrage von 517,870 Millionen Mark laufen, davon kommen 484,500 Millionen auf die 24 Gegenseitigkeitsvereine (nit 271,953 Millionen Mark gegen feste Prämien) und 33,370 Millionen auf die 6 anderen Unternehmer. Rückversicherungen finden sich nur bei den Gegenseitigkeitsvereinen. Von dem Gesamtumfange der Versicherungen dieser Vereine waren 3,775 Millionen Mark in Rückdeckung übernommen und 0,833 Millionen Mark in Rückdeckung weiter gegeben, sodaß sie für eigene Gefahr 483,667 Millionen Mark versichert hatten. Die Rückversicherung spielt hiernach bei der Viehversicherung nur eine untergeordnete Rolle.

Die Nettoprämieneinnahme aller Unternehmungen, d. h. die im Laufe des Jahres von den Versicherten eingenommenen Beträge nach Abzug der Rückversicherungsprämien, hat 10293301 ، $/$ betragen $(8072683$ N Vorprämien und feste Prämien, 2256707 .N Nachschußprämien und 36089 Al . Rückversicherungsprämien, Tab. III 5).

Als Schäden für eigene Rechnung ohne Regulierungskosten, aber unter Berücksichtigung des Zuwachses oder der Abnahme der Schadenreserve und unter Einschluß des Erlöses für verwertetes Vieh sind 9995548 dl zu verrechnen gewesen.

Wie sich die Versicherungsprämien und Schäden auf die verschiedenen Versicherungsmodalitäten, die Vieharten und deren Stückzahl verteilen, hat auch für 1903 nur unvollständig ermittelt werden können. Immerhin erscheinen die erzielten Ergebnisse wertvoll genug, um hier mitgeteilt zu werden; zumal sie einen Schluß auf den ganzen Versicherungsbestand wohl zulassen und auf manche Frage eine zuverlässige Antwort geben können. $\mathrm{Zu}$ beachten ist bei den folgenden Zahlen aber, daß der Anteil der Rückversicherer nirgends abgesetzt ist, ebensowenig sind bei den Prämien die Rabatte und bei den Schäden der Erlös ausgeschieden. Namentlich der letztgenannte Umstand ist bei der Beurteilung der Schäden von der größten Bedeutung, er schließt z. B. einen Vergleich der Schäden mit den Prämien vollständig aus, und er ist die Ursache, wenn auf 1000 cll Versicherungssumme gelegentlich mehr Schäden kommen als Prämien. Die 
Prämien sind die Einnahmen im Geschäftsjahr und die Schäden sind die im Geschäftsjahr entstandenen. Die in der umstehend folgenden Übersicht gegebenen Zahlen beruhen auf denselben Grundlagen wie Tabelle III 2, nur sind alle diejenigen Unternehmungen fortgelassen, bei denen nicht alle für die Übersicht erforderlichen Zahlen zu ermitteln waren. Die Annäherung der hier gegebenen Zahlen an die in Tabelle III 2 enthaltenen ist aber, wie man leicht sieht, wenigstens bei der Viehlebensversicherung eine recht befriedigende.

Die in Tabelle III 3 zum Vergleiche des Berichtsjahrs mit dem Vorjahr aufgeführten Zahlen sind auch dieses Mal bei den Versicherungssummen noch nicht streng vergleichbar, weil bei einigen Unternehmungen nicht der Betrag der überhaupt in Kraft gewesenen Versicherungen, sondern der Betrag der am Ende des Jahres laufenden Versicherungen eingestellt werden mußte. Sieht man hiervon ab, so ergibt sich für die Gegenseitigkeitsvereine, daß die Versicherungssummen von einem Geschäftsjahre zum andern um rund 91,19j Millionen Mark oder 20,3 Prozent, die Prämieneinnahme um rund 1,763 Millionen Mark oder 21,4 Prozent, und die Schadenzahlungen (ohne Abzug des Erlöses) um rund 1,609 Millionen Mark oder 19,9 Prozent zugenommen haben. Da im vorigen Jahre nicht dieselben Unternehmungen bearbeitet worden sind, so können zum Vergleiche nur die Verhältniszahlen herangezogen werden. Im Vorjahre betrug die $\mathrm{Zu}-$ nahme der Versicherungssumme 10,0 Prozent, der Prämieneinnahme 8,4 Prozent und der Schadenzahlungen 16,2 Prozent. Alle diese Zahlen bleiben also beträchtlich hinter denen des Berichtsjahrs zurück.

Vergleicht man die für eigene Rechnung behaltenen Versicherungssummen mit den Prämien und Schäden (III 1 und 3), nachdem man von den Schäden den Erlös (III 4) abgesetzt hat, was allerdings nur annähernd zu richtigen Ergebnissen führt, so findet man, daß im Jahre ${ }_{1903}$ bei den Gegenseitigkeitsvereinen auf 1000 , Nl Versicherungssumme 20,7 $\mathscr{H}$ Prämien und 15,0 $\lll$ Schäden kamen. Die in derselben Weise für die Unternehmungen der vorjährigen Statistik berechneten Werte geben für 190220,3 \& Prämien und 15,4 M. Schäden.

Von $1000 \mathscr{N}$. Prämien für eigene Rechnung war 1903 726 , auf Schäden nach Abzug des Erlöses zu verrechnen, nach den Erfahrungen der vorjährigen Statistik $756 \mathscr{M}$.

Die Gewinn- und Verlustrechnung (Tabelle IIl 4) stellt nur das Ergebnis des Geschäftsjahrs dar; der Gewinnvortrag aus dem Vorjahr ist fortgelassen, auch sind die Prämien- und Schadenreserven nicht mit ihrem ganzen Betrag am Anfange des Jahres in Einnahme und am Schlusse des Jahres in Ausgabe nachgewiesen, sondern es sind nur ihre Änderungen, die der Betrieb des Jahres zur Folge hatte, verrechnet, vgl. Tabelle III う.

Die gesamte Prämieneinnahme hat im Jahre $190310329390 \mathscr{M}$ betragen, davon kommen auf Vor- oder feste Prämien bei den Gegenseitigkeitsvereinen $7801633 \mathscr{N}$, bei den andern Unternehmungen $271050 \mathrm{c} /$. Die Nachschüsse bei den Gegenseitigkeitsvereinen beliefen sich auf $2256707 \mathscr{M}$ oder 28,9 Prozent der Vorprämien aller Vereine oder auf 43,6 Prozent der Vorprämien derjenigen Vereine, welche im Berichtsjahr überhaupt Nachschüsse erhoben haben.

Nebenleistungen der Versicherten kommen nur bei den Gegenseitigkeitsvereinen vor, sie haben im Jahre $1903357122 \mathscr{M}$ oder 3,6 Prozent der ganzen Prämieneinnahme oder 4, ,i Prozent der Vorprämien betragen, dabei ist zu bemerken, daß bei den meisten Versicherungsgesellschaften als satzungsmäßige Leistung der Mitglieder zum Reservefonds bestimmte Prozente der Vorprämie verrechnet und bei dieser nachgewiesen werden, also nicht bei den Nebenleistungen erscheinen.

Der Erlös aus verwertetem Vieh belief sich auf $2551224 \mathscr{H} \mathrm{d} . \mathrm{h}$. 25, 100 All Schäden für eigene Rechnung. Hierbei ist zu beachten, daß zum Teil nicht nur 


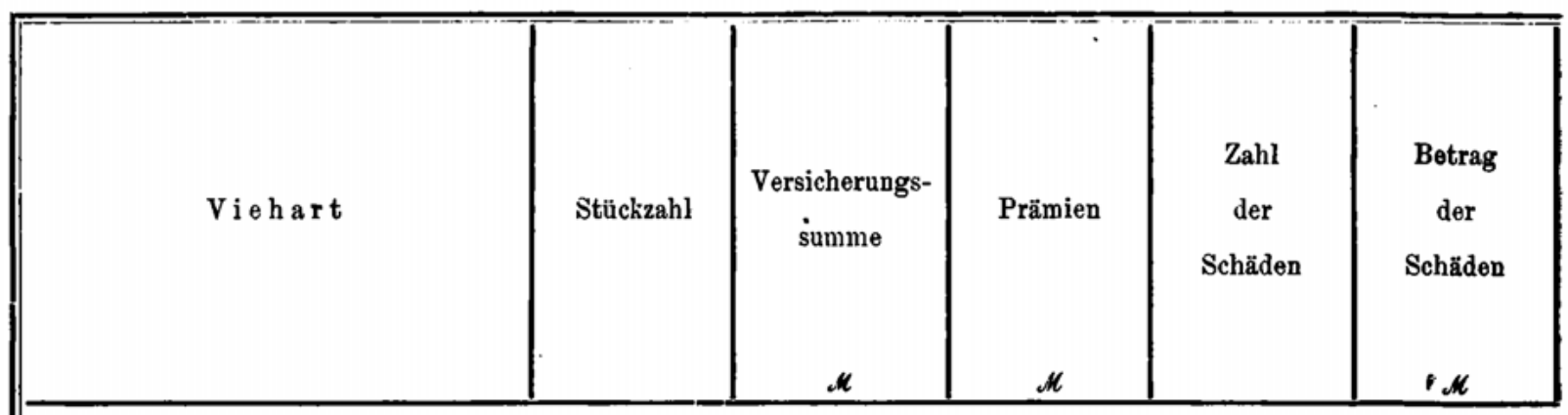

Viehversicherung

\begin{tabular}{|c|c|c|c|c|c|}
\hline Pferde (Maultiere, Esel) . . & 215389 & 149003651 & 5644650 & 10424 & $449 \times 348$ \\
\hline Rindvieh $\ldots \ldots \ldots \ldots \ldots$ & 523542 & 160920165 & $252780 x$ & 30860 & $3 \times 74473$ \\
\hline Schafe und Ziegen $\ldots \ldots \ldots \ldots$ & 20510 & 798878 & 11360 & 588 & 9436 \\
\hline 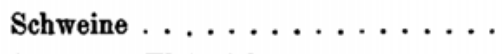 & I 241 995 & 120236400 & I 126579 & 30487 & $1237 \times 7 x$ \\
\hline Sonstiges Kleinvieh . . . . . & 4497 & 475963 & $225^{8}$ & 15 & 1501 \\
\hline Zusammen & 2005033 & 431435057 & 9312648 & 72374 & 8913020 \\
\hline
\end{tabular}

Viehlebensversicherung

Pferde (Maultiere, Esel) . . . . . . .

Rindvieh .............

Schafe und Ziegen .........

Schweine . . . . . . . .

Sonstiges Kleinvieh .........

Znsammen ...

\begin{tabular}{r|r|r|r|r|}
212059 & 146422718 & 5593089 & 10341 & 4455722 \\
129867 & 39102414 & 1232621 & 4797 & 1199530 \\
2035 & 68509 & 6027 & 162 & 5214 \\
163633 & 10220456 & 589450 & 9688 & $464 \times 42$ \\
100 & 13776 & 567 & 5 & 522 \\
\hline 507694 & 195827873 & 7421754 & 24993 & 6125130
\end{tabular}

Schlachtviehversicherung

\begin{tabular}{|c|c|c|c|c|c|}
\hline Pferde (Maultiere, Esel) $\ldots \ldots \ldots$ & - & - & - & & - \\
\hline Rindvieh $\ldots \ldots \ldots \ldots \ldots$ & 334696 & $10155^{\circ} 150$ & 1235715 & 25777 & I $92224 \mathrm{I}$ \\
\hline Schafe und Ziegen $\ldots \ldots \ldots \ldots$ & 18110 & 684754 & 5064 & 422 & 4034 \\
\hline Schweine $\ldots \ldots \ldots \ldots \ldots$ & 889270 & 89561812 & $48586 \mathrm{I}$ & 16774 & $487 \times 41$ \\
\hline Sonstiges Kleinvieh $\ldots \ldots \ldots \ldots$ & 4397 & $462 \times 87$ & I 691 & 10 & 979 \\
\hline Zusammen . . . & J 246473 & 192258903 & 1728331 & 42083 & 2414395 \\
\hline
\end{tabular}

\begin{tabular}{|c|c|c|c|c|c|}
\hline \multirow{6}{*}{$\begin{array}{l}\text { Pferde (Maultiere, Esel) } \ldots \ldots \ldots \ldots \\
\text { Rindvieh } \ldots \ldots \ldots \ldots \\
\text { Schafe und Ziegen } \ldots \ldots \ldots \ldots \ldots \ldots \\
\text { Schweine } \ldots \ldots \ldots \ldots \ldots \ldots \\
\text { Sonstiges Kleinvieh } \ldots \ldots \ldots \ldots \ldots \ldots\end{array}$} & \multirow{6}{*}{$\begin{array}{r}3330 \\
58979 \\
365 \\
189092 \\
-\quad\end{array}$} & \multirow{6}{*}{$\begin{array}{r}2580933 \\
20267601 \\
45615 \\
20454132 \\
-\end{array}$} & Sonstige & \multicolumn{2}{|c|}{ Viehversicherung } \\
\hline & & & $5 \times 56 \times$ & 83 & 35626 \\
\hline & & & 59465 & 286 & 52702 \\
\hline & & & 269 & 4 & 188 \\
\hline & & & 51268 & 4025 & 285888 \\
\hline & & & - & - & - \\
\hline Zusammen ... & 251766 & 43348281 & 162563 & 4398 & 374404 \\
\hline
\end{tabular}


Viehversicherung

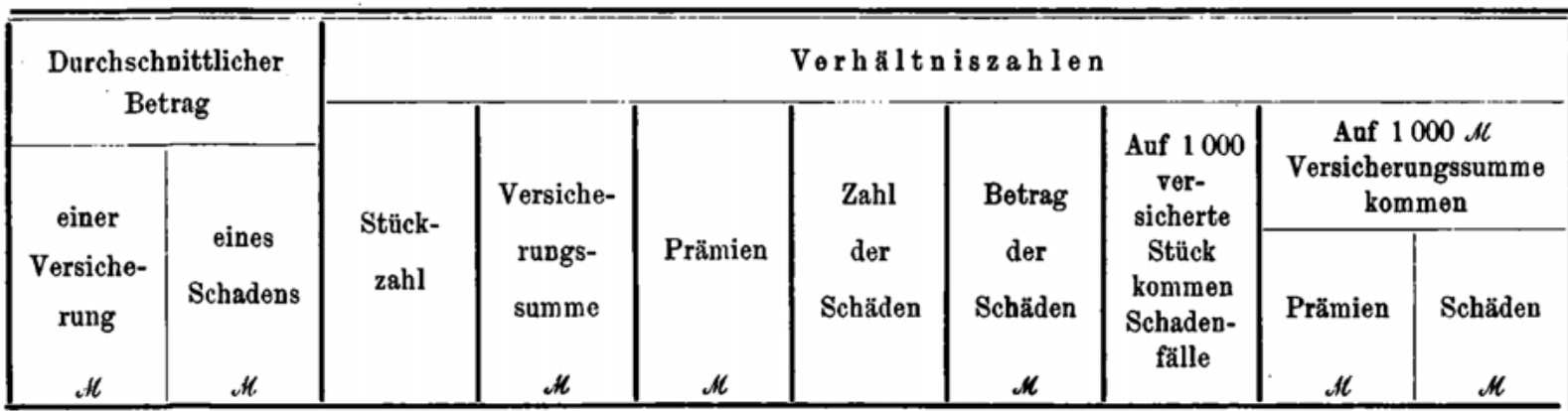

insgesamt

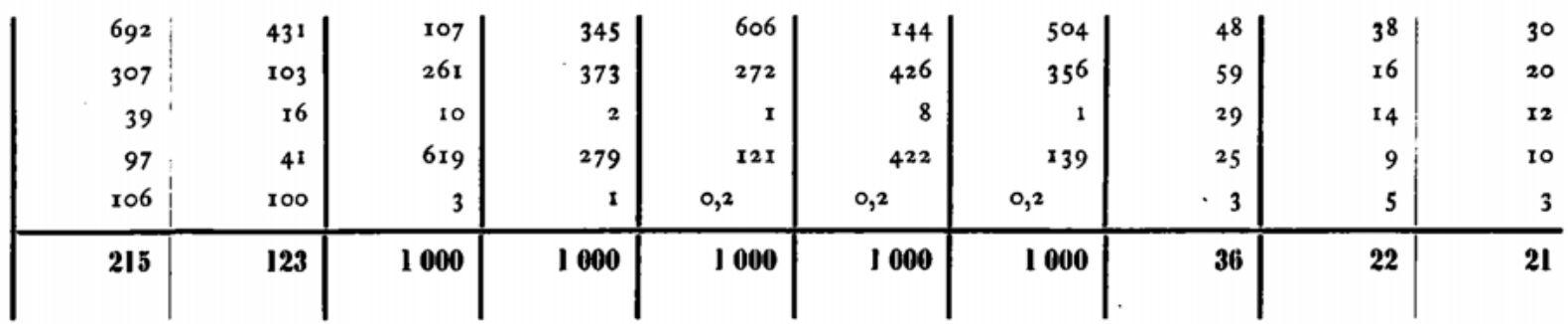

(24 Gegenseitigkeitsvereine)

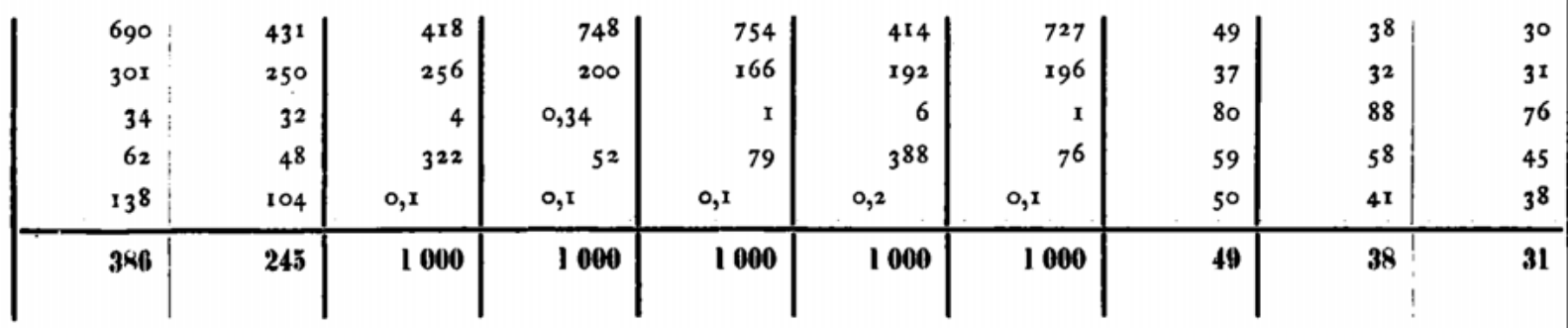

(8 Unternehmungen)

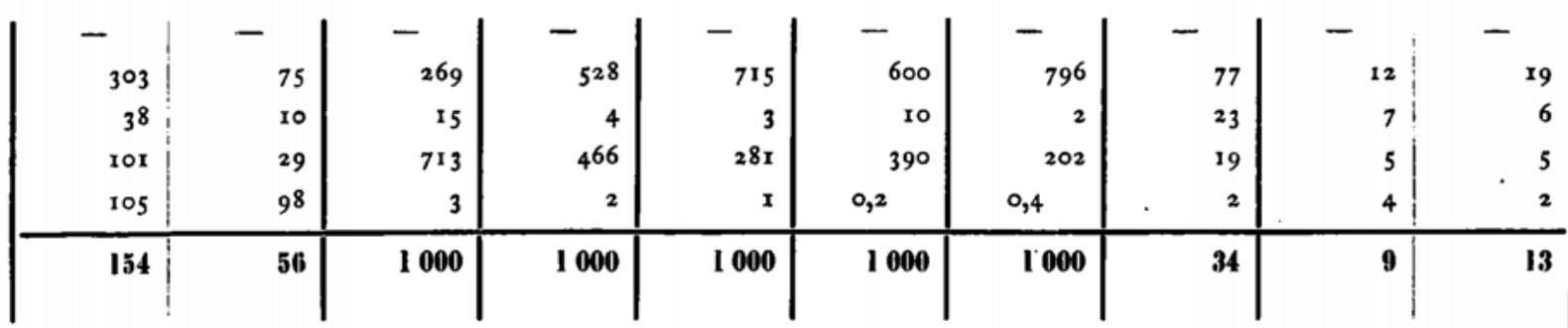

(8 Unternehmungen)

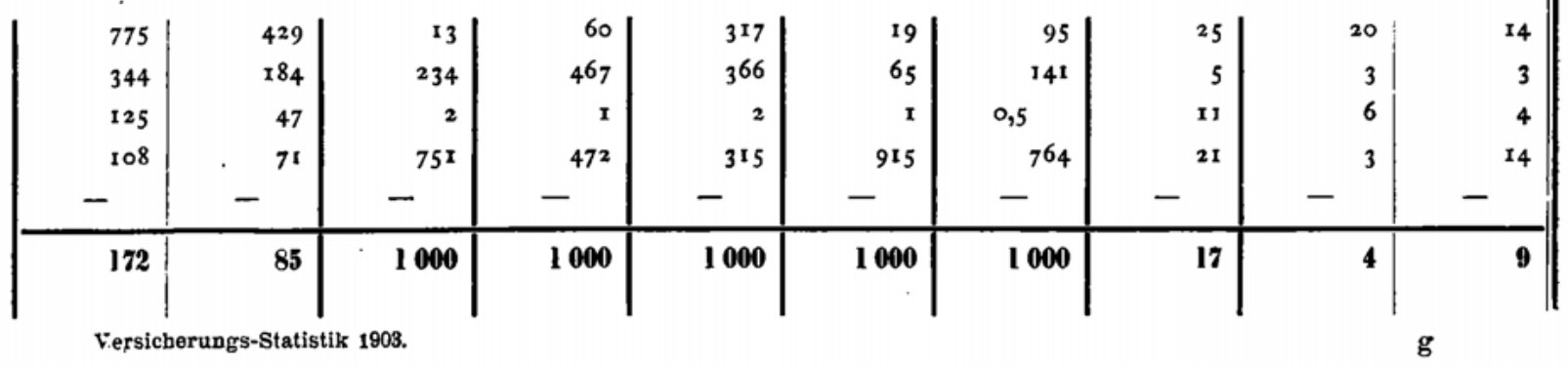


der wirklich erzielte Erlös oder der bedingungsgemäß gestattete Mindesterlös in Rechnung gestellt ist, sondern auch des öftern, insbesondere bei Versicherungen gegen Minderwert, der Unterschied zwischen Versicherungssumme und gezahltem Schadenbetrage.

An Regulierungskosten wurden $201256 \mathscr{N}$ oder 2,1 $/$ auf je $100 \mathscr{M}$ Schäden einschließlich Erlös gezahlt; an eigenen Verwaltungskosten und Steuern endlich 2482351 “ 1 . oder 24,5 Prozent der Prämien für eigene Rechnung (Tabelle III $5 \mathrm{Sp}$. 7).

Eine Schadenreserve aus dem Vorjahre kommt nur bei den Gegenseitigkeitsvereinen in Betracht, sie belief sich auf $274037 \mathscr{M}$, und die Schadenreserve am Ende des Berichtsjahrs betrug $309982 \mathscr{M}$ (Tabelle III 5). Die Schadenzahlungen des Geschäftsjahrs lassen sich zwar in Zahlungen für Schäden der Vorjahre und für solche des Geschäftsjahrs zerlegen, es ist aber nicht zu ersehen, ob alle Schäden der Vorjahre berichtigt wurden, oder ob von diesen noch einzelne mit in das Jahr 1904 übernommen werden mußten. Ist dies der Fall, so handelt es sich doch wohl nur um Ausnahmefälle, sodaß man annehmen darf, es sei aus der Schadenreserve ein kleiner Betrag, rund $36000 \mathscr{N}$, erspart worden. Jedenfalls war die Schadenreserve hoch genug bemessen, und es darf hervorgehoben werden, daß bei keinem einzigen Vereine die Zahlungen für Schäden der Vorjahre den Betrag der Schadenreserve überstiegen haben.

Die Bilanz Aus der Bilanz für r9o3, die hier nur für die Gegenseitigkeitsvereine gegeben wird, mögen folgende Posten angeführt werden:

Noch nicht eingezahltes Garantiekapital . x ro o00 “th Kapitalanlagen . . . . . . . . . r 959580, Forderungen ............... 3620328,

Kassenbestand ............. 106979 "

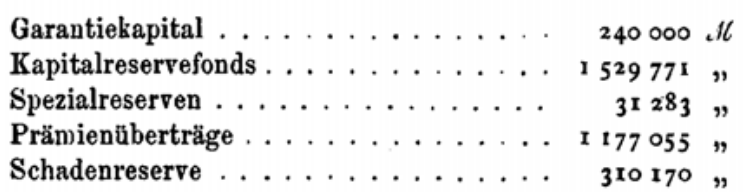

Die Bilanz schließt für alle Vereine zusammen mit $5881975 \mathscr{N}$ ab. Davon kommen bei den Aktiven 1,9 Prozent auf noch nicht eingezahltes Garantiekapital, 33,3 Prozent auf Kapitalanlagen, 61,6 Prozent auf Forderungen, 1,8 Prozent auf Kassenbestand und 1,4 Prozent auf die übrigen Aktiva; bei den Passiven 4,1 Prozent auf Garantiekapital, 26,0 Prozent auf Kapitalreservefonds, 0,5 Prozent auf Spezialreserven, 20,0 Prozent auf Prämienüberträge, 5,3 Prozent auf Schadenreserve und 44,1 Prozent auf die übrigen Passiva.

Die Kapitalanlagen bestehen zu 80,5 Prozent in Wertpapieren, zu 9,9 Prozent in Grundbesitz, zu 7,6 Prozent in Hypotheken und zu 2,0 Prozent in sonstigen Werten. Im Vergleiche mit dem Bestand am Ende des Vorjahrs waren im Besitze der Gegenseitigkeitsvereine Ende $1903 \mathrm{mehr}$ vorhanden an Grundbesitz $68913 \mathcal{N}$, an Hypotheken $42967 \mathscr{M}$, an Wertpapieren $76848 \mathscr{M}$ und an sonstigen Anlagen $33894 \mathscr{M}$. Die Forderungen bestehen bei der Gesamtheit zu 82,8 Prozent in Rückständen der Versicherten (einschl. der noch nicht gezahlten Nachschüssse); ins Gewicht fallen bei einigen Gesellschaften auch die Ausstände bei Agenten. Nur bei zwei Vereinen sind fast keine derartigen Rückstände vorhanden, ihre Forderungen sind fast ausschließlich Guthaben bei Banken.

Die Kapitalreservefonds haben gegen die Bilanz für Ende r9o2 um $296050 \mathscr{M l}$ oder um 24,0 Prozent zugenommen, dagegen sind die Spezialreserven um $122023 \mathbb{A l}$ oder 79,6 Prozent zurückgegangen.

Die vorstehenden Angaben beziehen sich sämtlich auf den gesamten Geschäftsbetrieb der genannten deutschen Viehversicherungsunternehmungen. Zur Beurteilung der Ausdehnung und des Umfanges des darin mit enthaltenen ausländischen Geschäfts mögen folgende Nachrichten dienen.

Das ausländische Geschäft
Im Jahre 1903 haben wie im Vorjahre nur 4 Versicherungsvereine auch im Auslande Geschäfte abgeschlossen, davon je einer in Belgien, Dänemark, den Niederlanden, Norwegen und Schw€den, zwei in Österreich und drei in der Schweiz. Diese Vereine hatten am 
Ende des Jahres im Ausland eine Summe von $2929010 \mathscr{H}$ versichert; an Prämien haben sie im Berichtsjahre $117487 \mathscr{N}$. eingenommen und an Schäden sind $86908 \mathscr{M}$ gezahlt. Es ist nlso das ausländische Geschäft in der Viehversicherung nur ganz unbedeutend gewesen; es hat von der gesamten Versicherungssumme der Vereine zu Ende des Jabres der ausländische Teil 6,0 vom Tausend betragen, von der Prämieneinnahme 11,6 vom Tausend und von den Schäden 8,9 vom Tausend.

\section{Hagelversicherung.}

Von den im Deutschen Reiche im Jahre 1903 vorhanden gewesenen Hagelversicherungs- Die Unterunternehmungen unterstanden dem Aufsichtsamte 5 Aktiengesellschaften und 17 Gegenseitigkeitsvereine; von den letzteren sind hier 6 als von lokalem Charakter und geringer Bedeutung außer Betracht gelassen worden. Von den in der vorjährigen Statistik aufgeführten Gegenseitigkeitsvereinen ist die Allgemeine Deutsche Hagel-VersicherungsGesellschaft in Liquidation in Berlin jetzt fortgelassen worden, weil die Liquidation beendet ist, und die Hagel-Versicherungsbank für Deutschland von I867 auf Gegenseitigkeit in Berlin, weil ihr Geschäft von der Ceres aufgenommen worden ist.

Drei von den hier behandelten elf Gegenseitigkeitsvereinen betreiben auch die Feuerversicherung, nämlich die Greifswalder, die Meklenburgische und die Schwedter Hagelund Feuer-Versicherungs-Gesellschaft. Alle drei halten aber die beiden Versicherungszweige in ihren Büchern getrennt und geben eine vollständige Gewinn- und Verlustrechnung und Bilanz für die Hagelversicherung einerseits und für die Feuerversicherung andererseits. Diese vollständige Trennung ist auch hier beibehalten worden, sodaß sich die hier für diese Gesellschaften gegebenen Zahlen lediglich auf die Hagelversicherung beziehen, wie die für dieselben Gesellschaften bei Gruppe IV gegebenen Zahlen lediglich das Feuerversicherungsgeschäft betreffen.

Ausländische Hagelversicherungsgesellschaften haben, soweit bekannt, im Jahre r903 im Deutschen Reiche nicht gearbeitet.

Der Umfang des Geschäfts im Berichtsjahre wird durch folgende Zahlen bezeichnet. Umfang Es waren insgesamt 2419,403 Millionen Mark auf 464151 selbst abgeschlossene Ver- $\begin{gathered}\text { der Ver- } \\ \text { sicherung }\end{gathered}$ sicherungen versichert oder im Durchschnitte a 213 . \% auf eine Police.

Hierin sind 116,268 Millionen Mark enthalten, die zu 4865 Kollektiv- oder Gemeindeversicherungen gehören, welche in der vorstehenden Zahl der Versicherungen je nur als eine Versicherung gezählt sind, aber rund 343000 Teilnehmer hatten. Die durchschnittlich von einem Versicherten versicherte Summe stellt sich demnach bei der Gesamtheit auf 3178 . /l und bei den Gemeinde- und Kollektivversicherungen allein auf 339 , 1 .

Von den Versicherungen kommen auf die Aktiengesellschaften 1002,285 Millionen Mark und 217808 Policen oder $4602, / l$ im Durchschnitte für die Police und auf die Gegenseitigkeitsvereine 1417,118 Millionen Mark und 246343 Versicherungen oder 5753, A im Durchschnitte für die Police.

Die Rückversicherung hatte keine große Bedeutung. In Rückdeckung übernommen hatten drei der Aktiengesellschaften zusammen 49,s4t Millionen Mark und in Rückdeckung gegeben ihrer vier 2,710 Millionen Mark, sodaß die Aktiengesellschaften zusammen von 1052,129 Millionen Mark überhaupt übernommene Versicherungen $1049,+19$ Millionen für eigene Rechnung behielten. Die Gegenseitigkeitsvereine haben nichts in Rückdeckung genommen und nichts in Rückdeckung gegeben. 
Die Nettoprämieneinnahme, d. h. die im Laufe des Jahres von den Versicherten eingenommenen Prämien, vermindert um die ausgegebenen Rückversicherungsprämien, hat insgesamt betragen $24523199 \mathscr{M}$, wovon $10484968 \mathrm{dl}$ auf die Aktiengesellschaften und $14038231 \mathcal{M}$ auf die Gegenseitigkeitsvereine entfielen (Tabelle III 12).

Als Schäden für eigene Rechnung, ohne die Regulierungskosten, aber unter Berücksichtigung der Änderung der Schadenreserve am Anfange des Jahres gegen die am Schlusse des Jahres (vgl. Tabelle III 12 und 14), sind insgesamt $17766523 \mathscr{M}$ zu verrechnen gewesen, $5706526 \mathscr{N}$ bei den Aktiengesellschaften und $12059997 \mathscr{N}$ bei den Gegenseitigkeitsvereinen. Als jedenfalls nicht zu kleine Zahl der Schäden (weil bei drei Gesellschaften nicht die Zahl der Schäden, sondern die Zahl der Anmeldungen angegeben ist) darf für die Gesamtheit 65339 angenommen werden, nämlich 30814 für die Aktiengesellschaften und 34525 für die Gegenseitigkeitsvereine. Es kommen also auf den Schadenfall im Durchschnitte mindestens: bei der Gesamtheit $272 \mathscr{M}$, bei den Aktiengesellschaften $18 \tilde{\mathscr{N}}$ : und bei den Gegenșeitigkeitsvereinen $349 \mathscr{M}$.

Die Prämienüberträge und die Schadenreserve spielen nur eine ganz untergeordnete Rolle (vgl. Tabelle III 14), man kann daher, ohne einen irgendwie merklichen Fehler zu begehen, die Prämien und Schäden, wie sie in Tabelle III 12 verzeichnet sind, als für das Geschäftsjahr geltend betrachten und sowohl mit einander wie auch mit der Versicherungssumme in Beziehung bringen. Das gibt für die in der diesjährigen Statistik behandelten Unternehmungen folgende Zahlen.

Von 1000 N․ Prämien wurden für Schadenzahlungen verwendet:

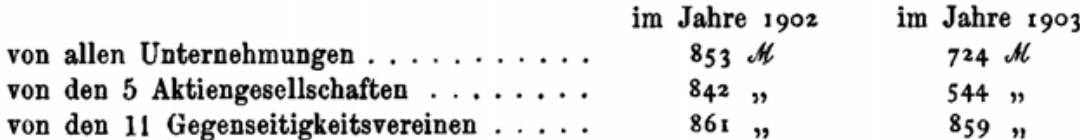

Auf 1000 , th Versicherungssumme kamen:

Prämien bei der Gesamtheit der Unternehmungen bei den 5 Aktiengesellschaften ....... bei den 11 Gegenseitigkeitsvereinen ..... Schäden bei der Gesamtheit der Unternehmungen bei den 5 Aktiengesellschaften ....... bei den 11 Gegenseitigkeitsvereinen......

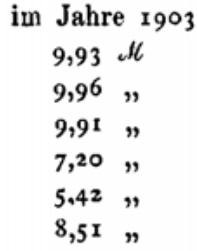

Vergleich des Berichtsjahrs mit dem Vorjahre

Eine Vergleichung des Geschäftsjahrs mit dem Vorjahre führt zu folgenden Ergebnissen (Tab. III 12).

Die Zahl der Versicherungen hat bei der Gesamtheit der Unternehmungen um 31279 oder 6,5 Prozent zugenommen, und zwar bei den Aktiengesellschaften um 19341 oder 7,9 Prozent und bei den Gegenseitigkeitsvereinen um 11938 oder 5,1 Prozent des Umfanges des Vorjahrs. Die Versicherungssummen sind insgesamt um 17,102 Millionen Mark oder 0, ? Prozent gestiegen, dabei steht eine Abnahme um 6,296 Millionen Mark oder 0,6 Prozent bei den Aktiengesellschaften einer Zunahme um 23,398 Millionen Mark oder 1,7 Prozent bei den Gegenseitigkeitsvereinen gegenüber. Die Nettoprämieneinnahme ist im Berichtsjahr um $392660 \mathscr{N}$ oder 1,6 Prozent gegenüber dem Vorjahre zurückgegangen, bei den Aktiengesellschaften ist sie zwar etwas größer gewesen (um $87954 \mathscr{M}$. oder 0,8 Prozent), bei den Gegenseitigkeitsvereinen aber wurden $480614 \mathscr{H}$ oder 3,3 Prozent weniger eingezogen. Eine größere Änderung zeigen allein die Schäden für eigene Rechnung, sie sind für die Gesamtheit der Unternehmungen um $3487447 \mathscr{M l}$ oder 16,4 Prozent geringer gewesen als im Vorjahre, bei den Aktiengesellschaften um 3051237 ath oder 34,8 Prozent, bei den Gegenseitigkeitsvereinen um 436210 . 11. oder 3,5 Prozent. 
Die in Tabelle III 13 gegebene Gewinn- und Verlustrechnung stellt nur das Ergebnis des Geschäftsjahrs dar; der Gewinnvortrag aus dem Vorjahr ist fortgelassen; auch Betriebssind die'sonstigen Überträge nicht mit ihrem vollen Betrage zu Anfang des Jahres in Einnahme und am Schlusse des Jahres in Ausgabe nachgewiesen, sondern nur ihre Änderungen, die der Betrieb des Geschäftsjahrs zur Folge hatte, verrechnet, vgl. Tab. III 14.

Dio Prämien haben bei den Aktiengesellschaften 10505333 ` $\%$ betragen, wovon für Rückversicherung 20365 ,// wieder ausgegeben wurden. Die Gegenseitigkeitsvereine haben 9582234 , $/$ Vorprämie und 4455997 ¿ N Nachschußprämie erhoben; die Nachschußprämie machte also 46,5 Prozent von der Vorprämie aus, oder wenn man die Greifswalder und die Neubrandenburger ausschließt, weil sie die Beiträge durch Umlage nach Bedarf aufbringen, 51,0 Prozent. Die von mehreren Gegenseitigkeitsvereinen durch die Gewinnund Verlustrechnung geführten „Rückversicherungsprämien“ sind hier fortgelassen; es handelt sich nämlich um Prämien für eine Nebenversicherung gegen etwaigen Nachschuß, die der Verein von seinen Mitgliedern einzieht, um sie vollständig an andere Versicherungsgesellschaften abzuführen, also um durchlaufende Posten, die den Geschäftsbetrieb nicht weiter berühren. Die Leistungen der Rückversicherer aus diesen Versicherungen sind bei den Nachschüssen eingestellt.

An Nebenleistungen hatten die Versicherten zu zahlen bei den Aktiengesellschaften $101656 \mathscr{M}$, das sind 0,97 Prozent der Prämien, und bei den Gegenseitigkeitsvereinen $1088194 \mathscr{N}$, das sind 7, i Prozent der gesamten Prämien oder 11,4 Prozent der Vorprämien. Die Nebenleistungen bestehen bei den Gegenseitigkeitsvereinen zum größten Teil $(806349, \mathscr{H})$ in satzungsmäßigen Beiträgen zum Reservefonds, die entweder nach der Höhe der Vorprämie oder der Entschädigung bemessen werden; setzt man diese ab, so bleiben 281845 $/ l$, das sind 2,0 Prozent der gesamten Prämien oder 2,9 Prozent der Vorprämien. Die von einigen Gegenseitigkeitsvereinen erhobenen Legegelder (Sicherheitsleistungen) sind dabei außer acht gelassen.

An Regulierungskosten sind insgesarnt bei allen Unternehmungen 1178553 \% oder $6,63, / l$ auf je $100 \mathscr{M}$. Schadenzahlung entstanden, bei den Aktiengesellschaften $569057 \mathscr{A}$ oder $9,9 i$ ¿th und bei den Gegenseitigkeitsvereinen 609496 , th oder 5,0s at auf je 100 , $1 /$ Schäden.

Für Verwaltung und Steuern sind (ohne den Anteil der Rückversicherer) ausgegeben von allen Unternehmungen zusammen 4954481 , th oder 20,2 Prozent, von den Aktiengesellschaften 2307167 \& oder 22,0 Prozent und von den Gegenseitigkeitsvereinen $2647314 \mathcal{N l}$ oder 18,s Prozent der Prämien für eigene Rechnung.

Das Geschäft des Jahres 1903 hat sämtlichen Aktiengesellschaften einen Überschuß gebracht, und zwar von zusammen 2546662 / ; bei den Gegenseitigkeitsvereinen weist Tabelle III 13 für drei Vereine einen Überschuß von zusammen $20452 \mathscr{M}$ nach.

Der Gewinn oder Verlust, wie ihn Tabelle III 13 ausweist, rührt aus dem Gesamtbetriebe des Geschäfts im Jahre 1903 her, und nicht aus dem eigentlichen Versicherungsbetrieb allein. Das ist namentlich bei den Aktiengesellschaften zu beachten, die beträchtliche Einnahmen aus ihrer Vermögensverwaltung ziehen. Will man den Gewinn oder Verlust aus dem Versicherungsbetrieb des Berichtsjahrs angenähert rein erhalten, so hat man diese auszuscheiden, das sind in Einnahme 658671, $\%$ und in Ausgabe 1300 \%, das Geschäftsergebnis verschlechtert sich also dadurch noch um $657371 \mathrm{Al}$, sodaß den fünf Aktiengesellschaften aus dem reinen Versicherungsgeschäfte des Jahres 1903 ein Gewinn von ungefähr 1889291 , entstanden ist.

Aus der Bilanz für 1903, die für die Áktiengesellschaften zusammen mit $38652301 \mathrm{~N}$ Die Bilanz und für die Gegenseitigkeitsvereine zusammen mit $4880338 \mathscr{A l}$ abschließt, mögen folgende Posten angeführt werden: 


\begin{tabular}{|c|c|c|c|}
\hline & 5 Aktiengesellschaften & 11 Gegenseitigke & itsvereine \\
\hline Nocht nicht eingezahltes Aktienkapital $\ldots \ldots \ldots$ & 21622800.4 & - & $\boldsymbol{\mu}$ \\
\hline Kapitalanlagen $\ldots \ldots \ldots \ldots \ldots \ldots$ & 14237417, & 3283730 & $\eta$ \\
\hline Forderungen $\ldots \ldots \ldots \ldots \ldots \ldots \ldots$ & 2649808 & 1472182 & $n$ \\
\hline Kassenbestand $\ldots \ldots \ldots \ldots \ldots \ldots \ldots \ldots \ldots \ldots \ldots \ldots$ & $135188 \%$ & 108841 & $"$ \\
\hline$\overline{\text { Aktienkapital } \ldots \ldots \ldots \ldots \ldots \ldots \ldots \ldots \ldots \ldots \ldots \ldots}$ & 28528500 & - & $\bar{\eta}$ \\
\hline Kapitalreservefonds . . . . . & I 246493 & 2919249 & $"$ \\
\hline Spezialreserven $\ldots \ldots \ldots \ldots \ldots \ldots$ & $6397051 \%$ & 392048 & $"$ \\
\hline Schadenreserven $\ldots \ldots \ldots \ldots \ldots \ldots \ldots \ldots \ldots \ldots \ldots$ & $11974 "$ & 205388 & $"$ \\
\hline
\end{tabular}

Bei den Aktiengesellschaften kommen danach von $1000 \mathscr{M}$ der Aktiva $559 \mathscr{H}$ auf noch nicht eingezahltes Aktienkapital, $368 \mathscr{M}$ auf Kapitalanlagen, $69 \mathscr{M}$ auf Forderungen und $3 \mathscr{M}$ auf Kassenbestand, von $1000 \mathrm{~N}$ der Passiven $738 \mathscr{A}$ auf Aktienkapital, $32 \mathscr{M}$ auf Kapitalreservefonds, $166 \mathscr{M}$ auf Spezialreserven, 0,3 $\mathscr{N}$ auf Schadenreserven und $64 \mathscr{M}$ auf andere Passiva. Bei den Gegenseitigkeitsvereinen dagegen $673 \mathscr{A l}$ auf Kapitalanlagen, $302 \mathscr{M}$ auf Forderungen, $22 \mathscr{M}$ auf Kassenbestand und 3 $\mathscr{H}$ auf sonstige Aktiva, $598 \mathscr{M}$ auf Kapitalreservefonds, $80 \mathrm{Al}$ auf Spezialreserven, $42 \mathrm{M}$. auf Schadenreserven und 280 Al auf andere Passiva.

Über die Kapitalanlagen gibt Tab. III 16 folgende Aufschlüsse. Von 1000 ¿ Kapitalanlagen kamen Ende r 903 auf

\begin{tabular}{|c|c|c|c|c|c|}
\hline & $\begin{array}{l}\text { Grund- } \\
\text { besitz }\end{array}$ & Hypotheken & $\begin{array}{l}\text { Wert- } \\
\text { papiero }\end{array}$ & $\begin{array}{l}\text { Darlehen auf } \\
\text { Wertpapiere }\end{array}$ & Wechsel \\
\hline$A$ & $21 M$ & $3^{81} \mathscr{M}$ & $540 \mathrm{~N}$ & 49 ath & $9 \mathscr{H}$ \\
\hline den Gegenseitigkei & $13 "$ & $174 \#$ & $720 "$ & - & $93 n$ \\
\hline
\end{tabular}

Ende $19{ }^{3}$ stand der Grundbesitz aller Unternehmungen mit 5677 weniger zu Buche als Ende 1902, der Bestand an Hypotheken ist im Vergleich mit der vorjährigen Bilanz um $647503 \mathscr{N}$ gefallen und der Bestand an Wertpapieren um $690180 \mathscr{N}$. gestiegen, an Darlehen auf Wertpapiere waren am Ende des Berichtsjahrs $677500 \mathrm{~N} / \mathrm{mehr}$ ausgegeben als am Ende des Vorjahrs.

\begin{tabular}{|c|c|c|c|c|c|c|}
\hline & $\begin{array}{c}\text { Von } 1000 \text { dl } \\
\text { Rückstände } \\
\text { bei } \\
\text { Versicherten }\end{array}$ & $\begin{array}{l}\text { Forderunge } \\
\text { Ausstände } \\
\text { bei } \\
\text { Agenten }\end{array}$ & $\begin{array}{r}\text { entfielen auf } \\
\text { Guthab } \\
\text { Bankhäusern }\end{array}$ & $\begin{array}{l}\text { f } \\
\text { ben bei } \\
\text { Ver- } \\
\text { sicherungs- } \\
\text { unternehmen }\end{array}$ & $\begin{array}{c}\text { Rück- } \\
\text { ständige } \\
\text { Zinsen und } \\
\text { Mieten }\end{array}$ & $\begin{array}{c}\text { Sonstige } \\
\text { Forde- } \\
\text { rungen }\end{array}$ \\
\hline en Aktiengesellschaften . . . . . &.$- \|$ & 40.11 & 875.11 & 26.4 & 58.11 & $-M$ \\
\hline en Gegenseitigkoitsvereinon . . . & $548 "$ & $67 "$ & $360 "$ & - & 10 , & I5, \\
\hline
\end{tabular}

Der Kapitalreservefonds steht bei den Aktiengesellschaften in der Bilanz für 1903 um $662206 \mathscr{M}$. oder um 34,z Prozent niedriger, bei den Gegenseitigkeitsvereinen dagegen um 402028 ull oder 16,0 Prozent höher als in der vorjährigen Bilanz. Die Spezialreserven erscheinen bei den Aktiengesellschaften um 81022 , $/$ oder um 1,3 Prozent und bei den Gegenseitigkeitsvereinen um 275085 H. oder um 41,2 Prozent niedriger als Ende r9oz, was zum Teil seinen Grund darin hat, daß bei einigen Vereinen beträchtliche Summen von den Spezialreserven zur Kapitalreserve übergeführt sind.

Über die Bedeutung des ausländischen Geschäfts der deutschen HagelversicherungsGeschäft unternehmungen, das in den vorstehenden Zahlen überall mit eingeschlossen ist, sind hier folgende Angaben zu machen.

Im ganzen haben vier Aktiengesellschaften (vornehmlich im Wege der Rückversicherung) und fünf Gegenseitigkeitsvereine auch im Auslande Geschäfte gemacht. In Frankreich, Italien, Rumänien und Ungarn war je eine Gesellschaft tätig, in Luxemburg zwei, in den Niederlanden und in RuPland je vier, in Österreich fünf. Die Versicherungssumme belief sich auf $\mathbf{6 , 0 4 1}$ Millionen Mark oder 22,7 vom Tausend der von allen Unter- 
nehmungen überhaupt versicherten Summe; die durchschnittliche Höhe einer selbst abgeschlossenen ausländischen Versicherung stellte sich auf 6308 c , war also beträchtlich höhèr als die durchschnittliche Versicherungssumme aus dem gesamten direkten Geschäfte. Die Bruttoprämieneinnahme aus dem ausländischen Geschäfte betrug 1208010 c/l oder 41,9 vom Tausend der gesamten Bruttoprämien und die Schäden 641435 /l oder 36,1 vom Tausend der gesamten Schäden.

\section{Gruppe IV, Feuerversicherung sowie die Versicherungen gegen Sturmschäden, Wasser- leitungsschäden und Einbruchdiebstahl.}

Von den deutschen Unternehmungen, die auf diesen Gebieten arbeiteten, unterstanden Die Unterin Jahre 1903 der Reichsaufsicht 43 Aktiengesellschaften und 30 Gegenseitigkeitsvereine. Diese Unternehmungen stellen aber keineswegs das gesamte private Feuerversicherungsgeschäft in deutscher Hand dar; es gibt vielmehr noch zahlreiche Vereine, welche wegen ihrer Beschränkung auf das Gebiet eines Bundesstaats dèr Aufsicht der Landesbehörden unterstehen, oder welche gemäß $\$ 3$ Abs. 2 des Versicherungsaufsichtsgesetzes der Landesaufsicht überwiesen worden sind.

Von den im Jahre 1903 unter Reichsaufsicht stehenden 30 Gegenseitigkeitsvereinen sind 11 in dem Folgenden nur nebenbei, nicht aber in den Tabellen behandelt, weil sie vermöge ihres geringen Versicherungsbestandes nur von untergeordneter Bedeutung sind, drei weitere sind fortgelassen, weil sie später der Aufsicht der Landesbehörden überwiesen worden sind. Von den verbleibenden 16 Gegenseitigkeitsvereinen wirken 13 lediglich in der Feuerversicherung; die anderen 3, nämlich die Hagel- und Feuer-Versicherungs-Gesellschaft auf Gegenseitigkeit zu Greifswald, die Mecklenburgische Hagel- und FeuerVersicherungs-Gesellschaft in Neubrandenburg und die Schwedter Hagel- und Feuer-Versicherungs-Gesellschaft, haben sich auch in der Hagelversicherung betätigt. Sie führen das Hagelversicherungsgeschäft aber, wie bereits bei Gruppe III erwähnt, vollständig von dem Feuerversicherungsgeschäfte getrennt, sodaß hier nur dieses Geschäft behandelt zu werden braucht. - Es sind in die vorliegende Statistik die im Vorjahre bearbeiteten 15 Vereine und außerdem die Feuer-Versicherungs-Gesellschaft für das Fürstentum Ratzeburg zu Schönberg, gegründet 1831, aufgenommen.

Von den 43 Aktiengesellschaften betrieben 27 die Feuerversicherung neben anderen Zweigen, 6 die Feuerversicherung als alleiniges Geschäft, die übrigen 10 befaßten sich nicht mit der Feuerversicherung, sondern - soweit die Versicherungszweige der Gruppe IV in Betracht kommen - nur mit der Sturm- und Wasserleitungsschädenversicherung und besonders mit der Einbruchdiebstahlversicherung, zwei von ihnen auch mit der Fahrraddiebstahlversicherung. $\mathrm{Zu}$ den in der vorjährigen Statistik bearbeiteten 42 Aktiengesellschaften ist die im Jahre 189 r gegründete Stuttgarter Mit- und Rückversicherungs-Gesellschaft zu Stuttgart hinzugekommen, die im Berichtsjahre den Betrieb der Sturmschäden-, der Wasserleitungsschäden- und der Einbruchdiebstahlversicherung aufgenommen hat.

Von ausländischen Versicherungsunternehmungen, die sich mit den zu Gruppe IV gehörigen Versicherungszweigen beschäftigten, unterstanden der Reichsaufsicht in Jahre 1903 insgesamt 45. Ein beträchtlicher Teil davon hat nur ein ganz unbedeutendes $\mathrm{Ge}-$ schäft meist mit starker örtlicher Begrenzung, sodaß es angezeigt erschien, die Verhältnisse dieser Gesellschaften hier nicht näher darzustellen; es wurden grundsätzlich diejenigen ausgeschieden, deren Bruttoprämieneinnahme aus dem deutschen Geschäfte den Betrag von 100000 N nicht, oder doch nicht erheblich überstiegen hat. Daher enthält diese Statistik nur Angaben über 29 ausländische Versicherungsgesellschaften. Von 
ihnen arbeiteten, soweit Versicherungszweige der Gruppe IV in Frage kommen, im Deutschen Reiche 20 nur in der Feuerversicherung, 5 (Nye danske Brandforsikrings Selskab, The Phönix Assurance Company Limited of London, Die Niederlande, Assicurazioni Generali und der Österreichische Phönix) in der Feuer- und Einbruchdiebstahlversicherung und 4 (Niederländischer Lloyd, Schweizerische National-Versicherungsgesellschaft, Schweizerische Unfallversicherungs-Aktiengesellschaft und Zürich) allein in der Einbruchdiebstahlversicherung. . $\mathrm{Zu}$ den in der vorjährigen Statistik bearbeiteten Unternehmungen sind die folgenden fünf hinzugekommen, denen das $\mathrm{Jahr}$ der Gründung und das Jahr der Aufnahme des deutschen Geschäftsbetriebs beigefügt ist:

Belgische Allgemeine Feuer-Versicherungs-Aktien-Gesellschaft in Brüssel 1830, 1881; Alliance Assurance Company in London 1824, 1825;

Atlas Assurance Company Limited in London 1808, 1887;

The Royal Exchange Assurance Corporation in London 1720, 1833;

K. K. privilegierte Riunione Adriatica di Sicurtà in Triest 1838, 1842.

Bei den folgenden Ausführungen sowie bei der Betrachtung der Tabellen ist, soweit es sich um Feuerversicherung handelt, im Auge zu behalten, $d a B$ es sich stets um die Gesamtheit der Versicherungen handelt; eine Trennung etwa in landwirtschaftliche, in einfache (bürgerliche) und in industrielle Risiken konnte nirgends vorgenommen werden; ebensowenig hat die Mobiliarfeuerversicherung von der Immobiliarfeuerversicherung geschieden werden können. Es wird nicht verkannt, daß hierin ein Mangel dieser Statistik liegt; solange aber von den Unternehmungen keine weitergehenden Auskünfte gefordert werden, als in den jetzt geltenden Rechnungsvorschriften enthalten sind, wird eine Verbesserung der Statistik nach dieser Richtung nicht zu ermöglichen sein.

Der Um-

fang des

Geschufts der

deutschen

Unternehmungen

Über den Umfang des Geschäfts der deutschen Unternehmungen in der Feuerversicherung Ende 1902 und Ende 1903 gibt Tabelle IV 1 Auskunft. Danach liefen an selbst abgeschlossenen Versicherungen einschließlich der davon in Rückdeckung gegebenen Teile

Ende $1902 \quad$ Ende 1903

bei den 30 Aktiengesellschaften . . . 7269674 Vers. über 73892 Mill. $\boldsymbol{~}$ h, 7474 101 Vers. über 77057 Mill. „k

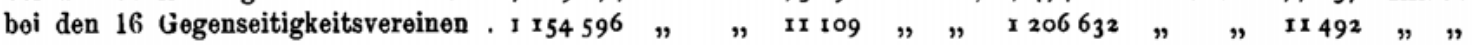
zusammen .. 8424270 Vers. über 85082 Mill. $\mathscr{M}, 8680733$ Ver8. über 88549 Mill $\mathscr{A C}$

Der Bestand Ende 1903 übertraf den zu Ende 1902 bei den Aktiengesellschaften um 204427 Versicherungen und 3165 Millionen Mark Versicherungssumme, bei den Gegenseitigkeitsvereinen um 52036 Versicherungen und 383 Millionen Mark, zusammen also um 256463 Versicherungen und 3548 Millionen Mark Versicherungssumme. Der durchschnittlich auf eine selbst abgeschlossene Versicherung (Mobiliar- und Immobiliarversicherung aller Risikengattungen) entfallende Betrag ist fast ungeändert geblieben, or belief sich bei den Aktiengesellschaften Ende rgoz auf 1016 s $\mathbb{H}$ und Ende r9o3 auf $10310 \mathrm{dl}$, bei den Gegenseitigkeitsvereinen auf $9622 \mathfrak{A l}$ und $9524 \mathrm{Nl}$, bei allen Unternehmungen zusammen auf $10100 \mathscr{A}$ und $10201, \mathscr{H}$.

Die Aktiengesellschaften hatten außerdem noch Versicherungen in Höhe von 8620 Millionen Mark zu Ende 1902 und in Höhe von 9957 Millionen Mark zu Ende 1903 in Rückdeckung genommen, sodaß bei ihnen die gesamte Bruttoversicherungssumme Ende r902 82512 und Ende 1903 87014 Millionen Mark betrug. In dieser Summe sind alle diejenigen Beträge doppelt gerechnet, welche von einer Gesellschaft, die in unserer Statistik behandelt wird, versichert und bei einer ebensolchen Gesellschaft in Rückdeckung gegeben sind; diese Beträge selbst $\mathrm{zu}$ bestimmen, ist nicht angängig. Dasselbe gilt von den unten angeführten Zahlen über die Gesamtversicherungssumme in der Einbruchdiebstahlversiche- 
rung. Der Anteil des mittelbaren Geschäfts am Gesamtgeschäft ist von 10,4 Prozent auf 11,4 Prozent gestiegen. - Die Gegenseitigkeitsvereine haben nichts in Rückdeckung übernommen.

Die Tabellen IV 2 und 3 zeigen, welchen Umfang bei den deutschen Aktiengesellschaften das inländische und das ausländische Feuerversicherungsgeschäft gehabt hat, auch hier sind die in Rückdeckung gegebenen Beträge eingeschlossen. Dabei ist zu beachten, daß das in Rückdeckung genommene Geschäft der Hanseatischen See- und Allgemeinen Versicherungsgesellschaft nicht in inländisches und ausländisches Geschäft getrennt werden konnte, weshalb dieses Geschäft ganz in Tabelle IV 2 aufgeführt und in Tabelle IV 3 fortgelassen ist.

Von den selbst abgeschlossenen Versicherungen kamen bei 30 Aktiengesellschaften

$$
\text { Ende } 1902
$$

Ende 1903

auf das inländische Geschäft. . . 6677989 Vers. über 6890 r Mill. «t, 6876295 Vers. über 7x 803 Mill. N

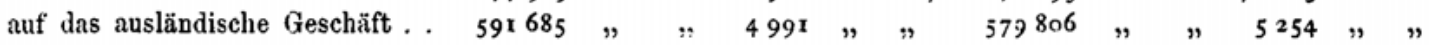

Das inländische unmittelbare Geschäft hat demnach um 198306 V'ersicherungen und 2902 Millionen Mark zugenommen; das ausländische um 6121 Versicherungen und 263 Millionen Mark.

Der durchschnittlich auf eine selbst abgeschlossene Versicherung entfallende Betrag ist bei den inländischen Versicherungen von 10318 , // auf 10442 , // gestiegen und bei den ausländischen Versicherungen von $8435 \mathrm{~N}$ auf $9062 \mathrm{~d}$.

Sieht man von der Hanseatischen See- und Allgemeinen Versicherungs-Gesellschaft ab, so hatten die übrigen 29 Aktiengesellschaften Ende ıgoz noch 1518 Millionen Mark im inländischen Geschäft und 6903 Millionen Mark im ausländischen Geschäft in Rückdeckung übernommen, Ende 1903 dagegen 169 כ Millionen Mark im inländischen und 7925 Millionen Mark im ausländischen Geschäfte.

Das gesamte inländische Geschäft der 29 Aktiengesellschaften erstreckte sich Ende I902 über 70414 Millionen Mark und Ende r9०3 über 73485 Millionen Mark, das gesamte ausländische (Geschäft Ende 1902 über 11859 und Ende 1903 über 13151 Millionen Mark. Der Anteil der mittelbaren Versicherungen an der Gesantheit betrug danach beim inländischen Geschäft 1902 2,2 Prozent und i9०3 2,3 Prozent, beim ausländischen dagegen 1902 58,2 Prozent und 1903 60,3 Prozent.

Endlich mag hier noch folgende Übersicht Platz finden, die über das Verhältnis des inländischen und des ausländischen Feuerversicherungsgeschäfts der 29 Aktiengesellschaften zu dem Gesamtgeschäft Aufschluß gibt, je nachdem es sich um selbst abgeschlossene, um in Rückdeckung übernommene Versicherungen oder un überhaupt abgeschlossene Versicherungen handelt.

\begin{tabular}{|c|c|c|c|c|c|c|}
\hline \multirow{2}{*}{$\begin{array}{c}\text { Von je } 1000 \mathscr{M} \\
\text { Versicherungssumme } \\
\text { kamen in der } \\
\text { Feuerversi cherung } \\
\text { bei } 29 \text { deutschen Aktien- } \\
\text { gesellschaften }\end{array}$} & \multicolumn{2}{|c|}{$\begin{array}{c}\text { bei den } \\
\text { selbst abgeschlossenen } \\
\text { Versicherungen }\end{array}$} & \multicolumn{2}{|c|}{$\begin{array}{l}\text { bei den in Räck- } \\
\text { deckung übernommenen } \\
\text { Versicherungen }\end{array}$} & \multicolumn{2}{|c|}{$\begin{array}{c}\text { bei den } \\
\text { überhaupt abgeschlossenen } \\
\text { Versicherungen }\end{array}$} \\
\hline & $\begin{array}{l}\text { auf das } \\
\text { inländische } \\
\text { Geschäft } \\
\text { HC }\end{array}$ & $\begin{array}{c}\text { auf das } \\
\text { ausländische } \\
\text { Geschäft } \\
\text { } U\end{array}$ & $\begin{array}{c}\text { auf das } \\
\text { inländische } \\
\text { Geschäft } \\
\text { „ル }\end{array}$ & $\begin{array}{c}\text { auf das } \\
\text { ausländische } \\
\text { Geschäft } \\
\text { „H }\end{array}$ & $\begin{array}{c}\text { auf das } \\
\text { inländische } \\
\text { Geschäft } \\
\mathscr{H}\end{array}$ & $\begin{array}{c}\text { auf das } \\
\text { ausländische } \\
\text { Geschäft } \\
\boldsymbol{M}\end{array}$ \\
\hline Ende $1902 \ldots \ldots \ldots$ & 933 & 67 & 180 & 820 & 856 & 144 \\
\hline Ende $1903 \ldots$ & 932 & 68 & 176 & 824 & 848 & 152 \\
\hline
\end{tabular}

Yon den Gegenseitigkeitsvereinen haben nur zwei (der Brandversicherungs-Verein der Preußischen Staatseisenbahn-Beamten und die Gothaer Feuerversieherungsbank) ausversicherungs-Statistik 1903. 
ländische Geschäfte und zwar in geringem Maße abgeschlossen. Beide zusammen hatten Ende r9o2 im Ausland 1447 Versicherungen über 39962000 ' $/$ und Ende r9o3 1414 Versicherungen über $39933000 \mathrm{~N}$. laufen.

In der Sturmschädenversicherung haben im Jahre r9o3 zwei Aktiengesellschaften gearbeitet, eine mehr als im Vorjahre, trotzdem war der Bestand an Versicherungen am Ende des Vorjahrs etwas größer als am Ende des Berichtsjahrs. Nach Tab. IV 4 liefen nämlich Ende 1902 insgesamt 2040 Policen über 345 Millionen Mark und Ende r 9031847 Policen über 303 Millionen Mark, der Rückgang des (Yeschäfts hat also 193 Policen und 42 Millionen Mark betragen. Von den Versicherungssummen kamen Ende 1902 96,0 Prozent und Ende 1903 ebensoviel auf das deutsche Geschäft. Rückversicherungen sind nicht übernommen worden.

Die durchschnittliche Versicherungssumme für eine Police betrug:

\begin{tabular}{|c|c|c|c|}
\hline & $\begin{array}{l}\text { beim Gesamt- } \\
\text { geschäfte }\end{array}$ & $\begin{array}{l}\text { beim inländischen } \\
\text { Geschäftc }\end{array}$ & $\begin{array}{c}\text { beim ausländischen } \\
\text { Geschäfte }\end{array}$ \\
\hline nde 1902 & $169035 \mu$ & $176269 u$ & $85736 M$ \\
\hline nde 1903. & $164 \times 25 \#$ & 170657 & $84479 "$ \\
\hline
\end{tabular}

In der Wasserleitungsschädenversicherung haben im Jahre rgo3 sieben Aktiengesellschaften gearbeitet, drei mehr als im Vorjahre. Nach Tab. IV 4 liefen Ende r 902 insgesamt 24938 Versicherungen über 893 Millionen Mark und Ende r903 30525 Policen über 1011 Millionen Mark, das Geschäft hat also um 5587 Policen und 118 Millionen Mark Versicherungssumme zugenommen. Von den Versicherungssummen kamen Ende 1902 99,5 Prozent und Ende 1903 99,3 Prozent auf das deutsche Geschäft. Rückversicherungen sind nicht übernommen worden.

Die durchschnittliche Versicherungssumme für eine Police betrug:

\begin{tabular}{|c|c|c|c|}
\hline & $\begin{array}{l}\text { beim Gesamt- } \\
\text { geschäfte }\end{array}$ & $\begin{array}{l}\text { beim inländischen } \\
\text { Geschäite }\end{array}$ & $\begin{array}{l}\text { beim ausländischen } \\
\text { Geschäfte }\end{array}$ \\
\hline vdo 1902 & $35828 \mu$ & $35798 \mu$ & $43753 \mu$ \\
\hline inde $1903 \ldots \ldots$ & $33131 \%$ & 33087 & 40826, \\
\hline
\end{tabular}

Sehr viel bedeutender ist das deutsche Geschäft in der Einbruchdiebstahlversicherung, an ihm haben sich 32 deutsche Aktiengesellschaften beteiligt, vier mehr als im Vorjahre. Nach den Tabellen IV 5 bis 7 kamen von den selbst abgeschlossenen Versicherungen

Ende $1902 \quad$ Ende 1903

auf das inländische Geschäft . . . . . . . 217051 Vers. über 353 I Mill. $\mathscr{N}, 274068$ Vers. über 4 313 Mill. $\mathscr{N}$

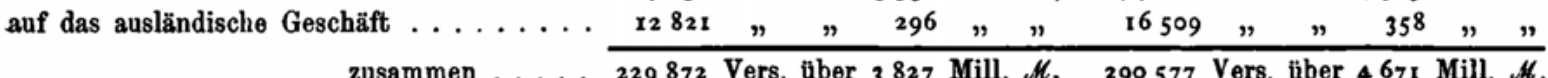

Dabei ist, wie überall in dieser Statistik, das Geschäft der Allianz und der Cölnischen Unfall-Versicherungs-Gesellschaft in der Fahrraddiebstahlversicherung mit eingeschlossen.

Das unmittelbare inländische Geschäft hat demnach um 57017 Versicherungen und 781 Millionen Mark, das ausländische um 3688 Versicherungen und 62 Millionen Mark zugenommen. Die durchschnittliche Höhe einer Versicherung ist bei dem inländischen Geschäfte von $16268 \mathscr{M}$ auf $15737 \mathscr{N}$ gefallen, beim ausländischen von 23087 ¿N auf $21685 \mathscr{N}$, bei dem Gesamtgeschäfte von $16648 \mathscr{N l}$ auf $16075 \mathscr{N}$. In Rückdeckung übernommen hatten diese Gesellschaften außerdem im inländischen Betrieb am Ende des Vorjahrs 265 und am Ende des Berichtsjahrs 625 Millionen Mark; sodaß bei ihnen die gesamte Bruttoversicherungssumme des deutschen Geschäfts Ende 19023796 Millionen Mark und Ende 19034938 Millionen Mark betrug. Im ausländischen Geschäfte twaren 
in Rückdeckung genommen Ende r902 189 und Ende r903 288 Millionen Mark; demnach ist die gesamte Bruttoversicherungssumme des ausländischen Geschäfts von 485 auf 646 Millionen Mark gestiegen. Der Anteil des indirekten Geschäfts am Gesamtgeschäft in der Einbruchdiebstahlversicherung belief sich danach beim inländischen Geschäft Ende 1902 auf 7,0 Prozent und Ende 1903 auf 12,7 Prozent; beim ausländischen Geschäfte dagegen auf 39,0 Prozent und 44,6 Prozent.

Alles in allem waren bei den 32 deutschen Gesellschaften am Ende des Vorjahrs 4281 und am Ende des Berichtsjahrs j) 584 Millionen Mark versichert.

Zur weiteren Erläuterung der Verhältnisse wird folgende Übersicht dienen.

\begin{tabular}{|c|c|c|c|c|c|c|}
\hline \multirow{2}{*}{$\begin{array}{c}\text { Von je } 1000 \AA \\
\text { Versicherungssumme } \\
\text { kamen in der } \\
\text { Einbruchdiebstahl- } \\
\text { versicherung } \\
\text { bei } 28 \text { deutschen Aktien- } \\
\text { gesellschaften }\end{array}$} & \multicolumn{2}{|c|}{$\begin{array}{c}\text { bei den } \\
\text { selbst abgeschlossenen } \\
\text { Versicherungen }\end{array}$} & \multicolumn{2}{|c|}{$\begin{array}{l}\text { bei den in Rück- } \\
\text { deckung übernommenen } \\
\text { Versicherungen }\end{array}$} & \multicolumn{2}{|c|}{$\begin{array}{l}\text { bei den } \\
\text { überhaupt abgeschlossenen } \\
\text { Versicherungen }\end{array}$} \\
\hline & $\begin{array}{l}\text { auf das } \\
\text { inländische } \\
\text { Geschäft } \\
\text { ฯ }\end{array}$ & $\begin{array}{c}\text { auf das } \\
\text { ausländische } \\
\text { Geschäft } \\
\text { H }\end{array}$ & $\begin{array}{l}\text { auf das } \\
\text { inländische } \\
\text { Geschäft } \\
\mathscr{N}\end{array}$ & $\begin{array}{l}\text { auf das } \\
\text { ausländische } \\
\text { Geschäft } \\
\qquad \Lambda\end{array}$ & $\begin{array}{c}\text { auf das } \\
\text { inländische } \\
\text { Geschäft } \\
\qquad \hbar\end{array}$ & $\begin{array}{c}\text { auf das } \\
\text { ausländische } \\
\text { Geschäft } \\
\mathscr{M}\end{array}$ \\
\hline Ende $x_{902} \ldots \ldots$ & 923 & 77 & $5^{84}$ & 416 & 887 & 113 \\
\hline Ende $1903 \ldots$ & 923 & 77 & 685 & 315 & 884 & 116 \\
\hline
\end{tabular}

Es ist bereits oben bemerkt, daß von den ausländischen Versicherungsunternehmungen, welche im Deutschen Reiche die Feuerversicherung betreiben, in die vorliegende Statistik nur 25 aufgenommen sind. Hier soll nur ihr deutsches Geschäft kurz umschrieben werden.

Die 25 ausländischen Gesellschaften hatten im Deutschen Reiche Ende 1902 selbst abgeschlossene Feuerversicherungen in Höhe von 9510 Millionen Mark und Ende 1903 in Höhe von 9899 Millionen Mark laufen, in Rückdeckung waren zu denselben Zeitpunkten Versicherungen im Betrage von 1351 und von 1519 Millionen Mark übernommen; der gesamte Versicherungsbestand betrug also in der Feuerversicherung am Schlusse des Vorjahrs 10861 und am Schlusse des Geschäftsjahrs 11418 Millionen Mark. Die selbst abgeschlossenen Versicherungen haben im Jahre 1903 um 389 und die in Rückdeckung übernommenen Versicherungen um 168 Millionen Mark zugenommen. Sieht man von der Assicurazioni Generali und für Ende 1902 auch von der Norwich Union und der Skåne ab, weil hier die Zahl der Versicherungen nicht bekannt ist, so stellt sich die auf eine selbst abgeschlossene Versicherung durchschnittlich entfallende Versicherungssumme am Ende des Vorjahrs auf $7644 \mathscr{M}$ und am Ende des Berichtsjahrs auf $7527 \mathscr{M}$; sie ist also etwas kleiner als bei den deutschen Unternehmungen.

Der Anteil des indirekten Geschäfts am Gesamtgeschäft ist von 12,4 Prozent auf 13,3 Prozent gestiegen.

Von den ausländischen Gesellschaften, die im Deutschen Reiche in der Einbruchdiebstahlversicherung gearbeitet haben, kommen hier neun in Betracht, von diesen haben fünf lediglich unmittelbare, vier auch mittelbare Geschäfte gemacht. Die selbst abgeschlossenen Versicherungen beliefen sich Ende 1902 auf 221 Millionen und Ende 1903 auf 293 Millionen Mark; rechnet man die in Rückdeckung übernommenen Versicherungen hinzu, so erhält man für den Bestand am Schlusse des Vorjahrs 248 und für den Bestand am Schlusse des Berichtsjahrs 330 Millionen Mark. Der durchschnittliche Betrag einer selbst abgeschlossenen Versicherung stellt sich bei Ausschluß des Geschäfts der Assicurazioni Generali, für das die Zahl der Versicherungen nicht bekannt ist, am Ende des Vorjahrs auf $15159 \mathrm{dl}$ und am Ende des Berichtsjahrs auf $13589 \mathrm{dl}$, er ist also ebenfalls kleiner als bei den deutschen Gesellschaften. 
Die Bruttoprämieneinnahme im Geschäftsjahr und die im Geschäftsjahr entstandenen arämien Sohäden Bruttoschäden aller Arten von Risiken, abweichend von der vorjährigen Statistik jedoch a) der ohne Regulierungskosten, sind für die deutschen Unternehmungen in den Tabellen IV 10

deutsohen
Gesell- bis 12 und für das deutsche Geschäft der ausländischen Gesellschaften in den Tabellen IV 13 sobaften und 14 zusammengestellt.

In der Feuerversicherung haben danach im Jahre 190330 deutsche Aktiengesellschaften 17ã $867636 \mathscr{N}$ und 16 Gegenseitigkeitsvereine $29881131 \mathscr{N}$, alle diese Unternehmungen zusammen also $205748767 \mathrm{~K}$ an Prämien eingenommen, und es sind in diesem Jahre den Aktiengesellschaften Schäden ohne Regulierungskosten im Betrage von 99469244 « , den Gegenseitigkeitsvereinen solche im Betrage von $8768034 \mathscr{N}$, zusammen $108237278 \mathscr{N}$. entstanden. Diese Schäden können aber nicht zu den Prämien in Beziehung gesetzt werden, weil die Prämien nicht die. Prämien für das Geschäftsjahr, sondern die im Geschäftsjahr überhaupt eingenommenen sind, also auch die für künftige Jahre im voraus gezahlten Beträge mit enthalten, während die früher für das Berichtsjahr gezahlten fehlen. Auch erheben einige Gegenseitigkeitsvereine, um Nachschüsse zu vermeiden, Vorprämien, die den regelmäßigen Bedarf weit übersteigen, und führen den daraus erwachsenen Gewinn den Versicherten wieder zu (vergl. Tab. IV 26). Schon aus diesen Gründen können die Prämien auch nicht etwa zu den durchschnittlichen Versicherungssummen in Beziehung gebracht werden. Es kommt aber noch hinzu, daß die Versicherungssummen sich nur auf den Bestand am Schlusse des Jahres beziehen, also zahlreiche kurzfristige Risiken, wofür die Prämien mit eingestellt sind, nicht mit umfassen. Den Verhältniszahlen würde aber auch nur eine sehr zweifelhafte Bedeutung zukommen, da die fraglichen Größen nicht nach Risikogattungen getrennt werden können.

Bei den deutschen Aktiengesellschaften kommen von der Prämieneinnahme 65̃,7 Prozent auf das inländische und 34,3 Prozent auf das ausländische Geschäft, und von den inländischen Prämien kommen 97,2 Prozent auf selbst abgeschlossene und nur 2,8 Prozent auf in Rückdeckung übernommene Versicherungen, von den ausländischen Prämien dagegen 53,4 Prozent auf das unmittelbare und 46,6 Prozent auf das mittelbare Geschäft.

Von den Schadensummen kommen 63,3 Prozent auf den inländischen und 36,7 Prozent auf den ausländischen Betrieb, von den inländischen Schäden stammten 96,8 Prozent aus selbst abgeschlossenen und 3,2 Prozent aus in Rückdeckung übernommenen Versicherungen, von den ausländischen Schäden dagegen 49,6 Prozent aus dem unmittelbaren Geschäft und 50,4 Prozent aus dem Rückversicherungsgeschäfte.

Der durchschnittliche Betrag eines Schadens aus selbst abgeschlossenen Feuerversicherungen ohne Regulierungskosten stellte sich beim inländischen Geschäft auf $520 \mathscr{H}$, beim ausländischen auf $1197 \mathscr{M}$. Eine Versicherungsanstalt konnte die Zahl der ihr aus dem Rückversicherungsgeschäft entstandenen Schäden nicht angeben und eine andere konnte bei den in Rückdeckung genommenen Versicherungen die inländischen nicht von den ausländischen trennen. Schaltet man daher diese beiden Gesellschaften aus, so hat ein Schadenfall aus den in Rückdeckung übernommenèn Versicherungen durchschnittlich im inländischen Betriebe 59 N und im ausländischen Betriebe 144 , gekostet.

Bei den Gegenseitigkeitsvereinen hat der Schadenfall durchschnittlich $499 \mathrm{NH}$, im inländischen Geschäfte $499 \mathscr{M}$, im ausländischen (23 Fälle) $28, \mathbb{N}$ erfordert.

Die beiden Gesellschaften, die in der Versicherung gegen Sturmschäden gearbeitet haben, erzielten (Tab. IV 11) aus diesem Geschäft eine Prämieneinnahme von $88448 \varkappa$, wovon 98,9 Prozent aus dem deutschen Betriebe herrührten. An Schäden sind 355 mit $103848 \mathscr{M}$. (ohne Regulierungskosten) zu verzeichnen gewesen, davon stammten 334 mit $100562 \mathscr{M}$ aus deutschen Versicherungen, der durchschnittliche Betrag eines Schadens stellte sich demnach im Gesamtgeschäft auf 293 ، , im deutschen Betrieb auf 301 At und im ausländischen Betrieb auf 1 こ̌ $\mathfrak{A l}$. 
Die sieben Gesellschaften, die in der Versicherung gegen Wasserleitungsschäden gearbeitet haben, erzielten aus diesem Geschäfte (Tab. IV 11) eine Prämieneinnahme von 876810 \%, wovon 99,4 Prozent aus dem deutschen Betriebe herrührten. An Schäden sind 4198 mit 285510 « (ohne Regulierungskosten) zu verzeichnen gewesen, die ebenfalls fast ausschließlich aus deutschen Versicherungen stammten. Der durchschnittliche Betrag eines Schadens stellte sich auf 68 , $/$.

In der Einbruchdiebstahlversicherung (Tab. IV 12) haben 32 deutsche Aktiengesellschaften $4917476 \mathscr{H}$ an Prämien eingenommen und für 8062 Schäden 1114464 c/ zu verbuchen gehabt. Aus den vorhin bei der Feuerversicherung angegebenen Gründen ist eine Vergleichung der Prämien mit den Schäden und den Versicherungssummen auch hier nicht angängig.

Von der Prämieneinnahme kommen 87,2 Prozent auf das inländische und 12,8 Prozent auf das ausländische Geschäft, und von den inländischen Prämien kommen 90,0 Prozent auf selbst abgeschlossene Versicherungen und 10,0 Prozent auf in Rückdeckung übernommene Versicherungen, von den ausländischen Prämien dagegen 44,6 Prozent auf das unmittelbare und 55,4 Prozent auf das mittelbare Geschäft.

Der durchschnittliche Betrag eines Schadens stellte sich überhaupt auf 138 c $\%$ und zwar bei den selbst abgeschlossenen Versicherungen ini inländischen Geschäft auf 130 ¿ $/$. und im ausländischen Geschäft auf 354 , $/$; bei den in Rückdeckung übernommenen Versicherungen im inländischen Geschäft auf $133 \mathrm{~d}$ und im ausländischen Geschäft auf $135 \mathrm{Al}$.

Für das deutsche Feuerversicherungsgeschäft der 25 ausländischen Gesell- b) der ans. schaften gelten folgende Zahlen (Tab. IV 13). Die Prämieneinnahme belief sich auf ${ }_{\text {Gesell- }}^{\text {ländiscen }}$ 20878635 , $/$ und für Schäden waren $10721826 \mathscr{N}$ aufzuwenden. Von den Prämien schaften stammten 84,4 Prozent aus sclbst abgeschlossenen Versicherungen und 15,ij Prozent aus Rückversicherungen, von den Schäden 80,8 Prozent aus selbst abgeschlossenen Versicherungen und 19,2 Prozent aus in Rückdeckung übernommenen Versicherungen.

Das deutsche Einbruchdiebstahlversjcherungsgeschäft der hier in Betracht kommenden 9 ausländischen Gesellschaften, das im wesentlichen unmittelbar betrieben wird, zeigt sich auch hinsichtlich der Prämieneinnahme und der Schadenleistungen (Tab. IV 14) als wenig bedeutend. Die Prämieneinnahme hat nur 321713 , 1 l betragen, und es sind 593 Schäden in Höhe von $47821 \mathscr{N}$ im Geschäftsjahr entstanden.

Eine Vergleichung der Bruttoprämien und Schäden aus dem ausländischen Ge- Das ausschäfte der deutschen Gesellschaften mit denjenigen aus dem deutschen Geschäfte der $\begin{aligned} & \text { ländische } \\ & \text { Geschäft }\end{aligned}$ ausländischen Gesellschaften führt zu folgender Zusammenstellung:

\begin{tabular}{|c|c|c|c|c|c|}
\hline & $\begin{array}{l}\text { Ausländis } \\
\text { deutsch }\end{array}$ & $\begin{array}{l}\text { chäft đer } \\
\text { Ischaften }\end{array}$ & $\begin{array}{l}\text { Deutsches } \\
\text { ländisch }\end{array}$ & $\begin{array}{l}\text { it der aus- } \\
\text { lschaften }\end{array}$ & $\begin{array}{l}\text { deutschen } \\
\text { und das } \\
\text { deutsche }\end{array}$ \\
\hline & $\begin{array}{c}\text { Prämien } \\
\mathscr{A}\end{array}$ & $\begin{array}{c}\text { Schäden } \\
\star\end{array}$ & $\begin{array}{l}\text { Prämien } \\
\text { \&ll }\end{array}$ & $\begin{array}{c}\text { Schäden } \\
\mathbb{d}\end{array}$ & $\begin{array}{c}\text { Geschäft } \\
\text { der aus- } \\
\text { landischen }\end{array}$ \\
\hline Feuerversicherung $\ldots \ldots \ldots \ldots$ & 60403371 & 36531784 & $20 \$ 78635$ & 10721826 & Gesell- \\
\hline Versicherung gegen Sturmschäden ..... & $93^{8}$ & 3286 & - & - & schaften \\
\hline Versicherung gegen Wasserleitungsschäden & 5394 & 477 & - & - & \\
\hline Versicherung gegen Einbruchdiebstahl ... & 627356 & 162833 & 321713 & 47821 & \\
\hline zusammen . . & $37 \circ 59$ & 36037059 & 21200348 & 10769647 & \\
\hline
\end{tabular}

Es übertrifft also das ausländische Geschäft der deutschen Unternehmungen das deutsche Geschäft aller im Inland auf diesem Gebiet arbeitenden ausländischen Gesellschaften ganz bedeutend. Das kann aber auch nicht überraschen, da sich die deutsche Feuerversicherung und ihre Nebenzweige in fast allen wichtigeren Staaten der Welt zu beteiligen wissen. Es arbeiteten, um nur diejenigen Länder zu nennen, in denen deutsche 
Gesellschaften besonders zahlreich vertreten sind, im Jahre 1903 in den Niederlanden 25 Gesellschaften, in Dänemark 20, in Österreich 19, in der Schweiz 18, in Belgien und in Schweden je 16, in England 14, in den Vereinigten Staaten 13, in Rußland 11, in Frankreich 10, in Luxemburg und Norwegen je 9, in Chile, China und in der Türkei je 8 usw.

Die Rechnungs:-
legung be

Bei weitem die meisten deutschen Aktiengesellschaften, die auf Ver'sicherungsgebieten der Gruppe IV arbeiten, betreiben, wie schon bemerkt, neben ihrem Hauptgeschäfte noch andere Versicherungszweige. Dabei trennen sie die verschiedenen Geschäfte nicht immer Betriebe und die Kapitalerträge einerseits und die Verwaltungskosten, Steuern, Abschreibungen und Verluste aus der Vermögensverwaltung andererseits nur für das Gesamtgeschäft, auch lassen sich die "sonstigen“ Einnahmen und Ausgaben vielfach nicht nach Versicherungszweigen scheiden. Die oben bei der Besprechung der Unfall- und Haftpflichtversicherung (S. XXXVIII) mitgeteilten Gründe, weshalb das Aufsichtsamt einstweilen nicht auf einer vollständigen Zerlegung des Geschäfts nach Versicherungszweigen bestanden hat, sind auch hier ausschlaggebend gewesen, und wie dort, so sind auch hier zunächst den einzelnen Versicherungszweigen eigentümliche Sonder-Einnahmen und Sonder-Ausgaben für jeden Zweig ermittelt. Bei Gesellschaften der genannten Art ist dann nur eine Gewinn- und Verlustrechnung für das Gesamtgeschäft aufgestellt worden, in der an der Spitze der Einnahmen die Summe der Sonder-Einnahmen aller Versicherungszweige der Gesellschaft und an der Spitze der Ausgaben die Summe der Sonder-Ausgaben aller Zweige erscheint. Der Gleichförmigkeit wegen ist bei denjenigen Unternehmungen, welche lediglich in der Feuerversicherung tätig sind (in erster Linie also bei den Gegenseitigkeitsvereinen), oder welche für jeden Zweig eine vollständige Zerlegung aller Posten durchführen, bei jedem einzelnen Zweige ebenso verfahren, bei ihnen ist also die Summe der Sonder-Einnahmen und Ausgaben aller Zweige hier gleichbedeutend mit der Summe der Sonder-Einnahmen und Ausgaben der Feuerversicherung.

Bei Gruppe IV sind als Sonder-Einnahmen die Prämien des Geschäftsjahrs für eigene Rechnung (Jahreseinnahme nach Abzug der Ristorni und der verausgabten Rückversicherungsprämien), die Nebenleistungen der Versicherten, die Ersparnis aus der Schadenreserve des Vorjahrs und die Abnahme der Prämienüberträge zusammengefaßt und als SonderAusgaben die Zahlungen und Rückstellungen für Schäden (einschließlich Regulierungskosten), die im Geschäftsjahr entstanden sind, nach Abzug der Leistungen der Rückversicherer, aber einschließlich der Schadenermittelungskosten, die Leistungen für gemeinnützige Zwecke, der Zuschul. zur Schadenreserve des Vorjahrs und die Zunahme der Prämienüberträge. Gegenüber dem Vorjahr ist also insofern eine Vervollkommnung eingetreten, als jetzt die Änderung der Prämienüberträge bei den Sonder-Einnahmen und Ausgaben verrechnet wird, was in der vorjährigen Statistik noch nicht möglich war.

Bei den deutschen Unternehmungen in der Feuerversicherung betragen

\begin{tabular}{|c|c|c|}
\hline $\begin{array}{l}\text { bei der Gesamthoit } \\
\text { der Unternchmungen }\end{array}$ & $\begin{array}{l}\text { bei } 30 \text { Aktien- } \\
\text { gesellschaften }\end{array}$ & $\begin{array}{c}\text { bei } 16 \text { Gegenseitigkeits- } \\
\text { vereinen }\end{array}$ \\
\hline $132208488 \mu$ & $103793594 \mu$ & $28414894 \cdot N$ \\
\hline 70973417 & $62352451 "$ & 8620966, \\
\hline
\end{tabular}

die Summe der Sonder-Einnahmen ... die Summe der Sonder-Ausgaben ....

70973417 "

62352451 "

8414894 .h
$8620966, "$

Von je 1000 “॥. Sonder-Einnahmen der Feuerversicherung kamen

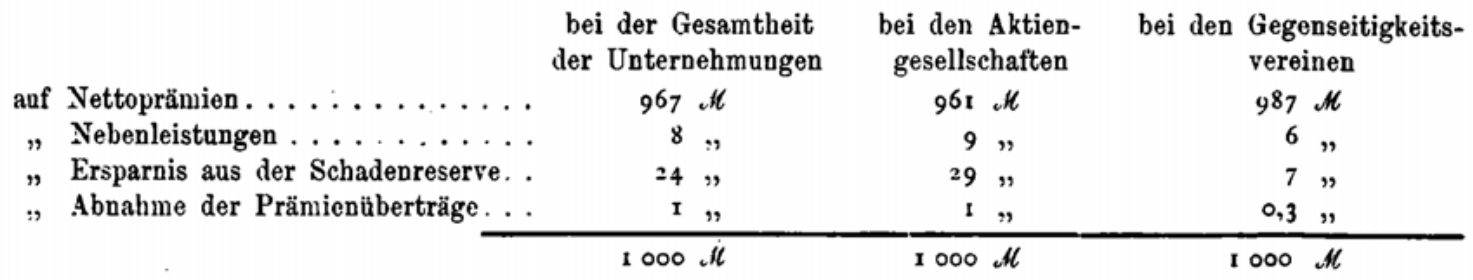


Von je 1000 .t/ Sonder-Ausgaben kamen

\begin{tabular}{|c|c|c|c|}
\hline . & $\begin{array}{l}\text { bei der Gesamthoit } \\
\text { der Unteruchmungen }\end{array}$ & $\begin{array}{l}\text { bei den Altion- } \\
\text { gesellschaften }\end{array}$ & $\begin{array}{l}\text { bei den Gegenseitigkeits } \\
\text { vereinen }\end{array}$ \\
\hline \multirow{6}{*}{$\begin{array}{l}\text { auf gezahlte Schäden . . . . . . . } \\
\text { " zurückgestellte Schäden . . . . . } \\
\text { " Leistungen für gem. Zwecke . . } \\
\text { " Zuschuß zur Schadenreserve . . } \\
\text { " Zunahme der Prämienüberträge }\end{array}$} & 737 ett & 733 ath & $755 \mathrm{~d}$ \\
\hline & $160 "$ & 168, & rto" \\
\hline & $23 "$ & $24 "$ & $16 "$ \\
\hline & 0,02, & 0,02, & - " \\
\hline & 80, & $75 "$ & Irg \\
\hline & 1000 ath & 1000 etl & $1000 \mathrm{AC}$ \\
\hline
\end{tabular}

Von den in Geschäftsjahr entstandenen Schadensummen wurden durchschnittlich 82,1 Prozent gezahlt, bei den Aktiengesellschaften 81,4 Prozent, bei den Gegenseitigkeitsvereinen 87,3 Prozent, der Rest der Schadenreserve überwiesen. - Von den Leistungen für gemeinnützige Zwecke beruhten bei den Aktiengesellschaften 61,5 Prozent und bei den Gegenseitigkeitsvereinen 85,5 Prozent auf gesetzlicher Vorschrift.

Aus der Vergleichung der Tab. IV 10 und IV 15 findet man, daß die Aktiengesellschaften von 175867636 , Al Bruttoprämieneinnahme für Feuerversicherung 76097988 «th oder 43,3 Prozent an Rückversicherungsprämien ausgegeben haben; die Gegenseitigkeitsvereine haben dagegen von 29811131 , th nur $1765821 \mathscr{A l}$ oder 5,9 Prozent für Rückversicherung gezahlt. Die Bruttoschäden und die Nettoschäden können in diesem Jahre nicht mehr in Beziehung gesetzt werden, weil in Tab. IV 10 alle Regulierungskosten ausgeschlossen sind, während in Tab. IV 15 die von den Gesellschaften für eigene Rechnung aufgewendeten Regulierungskosten eingeschlossen sind.

Zu der Sturmschäden-, der Wasserleitungsschäden- und der Einbruchdiebstahlversicherung ist hier folgendes zu bemerken:

\section{Es betrug}

die Summe der Sonder-Einnahmen .... die Summe der Sonder-Ausgaben ..... bei der Sturmschädenversicherung

(2 Aktiengesellschaften)

25794 ch

18854 ,

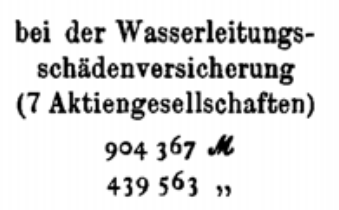
schädenversicherung

$904367 \mu$ 439563, bei der Einbruchdiebstahl-, versicherung

(32 Aktiengesellschaften)

$3626024 \mu$

I 200582 "

Von je 1000 Al der Sonder-Einnahmen kamen

auf Nettoprämien . . . . . . . . . .

" Nebenleistungen der Versicherten...

"Ersparnis aus der Schadenreserve . . .

" Abnahme der Prämienüberträge ....
$702 \mu$
$18 \%$
$278 "$,
$2000 \%$

$\begin{array}{r}2 \% \\ 18 \% \\ 2 \% \\ 2 \% \\ \hline \%\end{array}$

$936 \mathcal{M}$

ro

54 ,

$-"$

$\mathrm{I} 000 \mathrm{M}$
$951 \cdot \boldsymbol{H}$

27 "

18 "

4,

Von je 1000 . $l l$ der Sonder-Ausgaben kamen

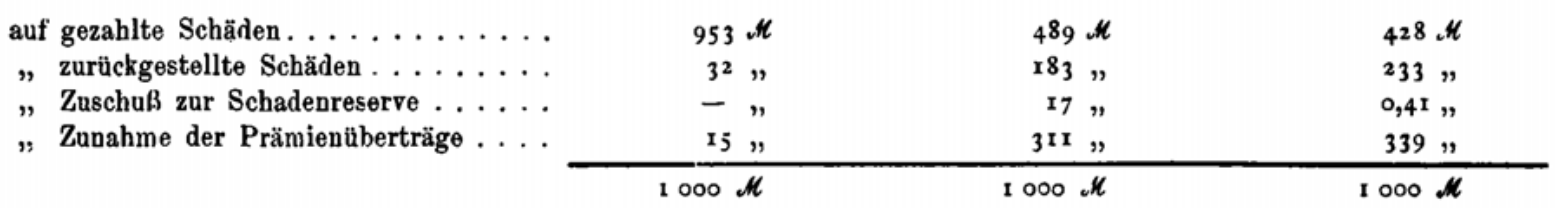

Von den im Geschäftsjahr entstandenen Nettoschäden wurden bei der Sturmschädenversicherung 96,8 Prozent, bei der Wasserleitungsschädenversicherung 72,8 Prozent und bei der Einbruchdiebstahlversicherung 64,7 Prozent noch im Geschäftsjahre beglichen, der Rest in Schadenreserve gestellt.

Aus der Vergleichung der Tab. IV 11 mit IV 16 folgt, daß von den $88448 \mathscr{M}$ betragenden Bruttoprämien für die Sturmschädenversicherung $70338 \mathscr{N}$ oder 79,5 Prozent für Rückversicherung ausgegeben wurden, daß dagegen in der Wasserleitungsschädenversicherung sich diese Ausgaben bei einer Bruttoprämieneinnahme von 876810 \&H. nur auf 
30496 , /l oder 3,5 Prozent stellte. Ebenso folgt aus der Vergleichung der Tab. IV 12 und 17, daß von der Bruttoprämie von $+917476 \mathrm{~d} /$ für die Einbruchdiebstahlversicherung 1469606 ، oder 29,9 Prozent für Rückversicherung ausgegeben wurden.

Die Gewinn- und Verlustrechnung soll nur das Ergebnis des Geschäftsjahrs darstellen, deshalb ist der Gewinnvortrag aus dem Vorjahre fortgelassen und es sind hier Reserven und Rücklagen wie schon bei der Ermittelung der Sonder-Einnahmen und Sonder-Ausgaben (Tab. IV 15 bis 17) nicht mit ihrem ganzen Betrag am Anfang des Jahres in Einnahme und am Schlusse des Jahres in Ausgabe nachgewiesen, sondern es sind nur ihre Änderungen, die der Geschäftsbetrieb des Jahres zur Folge hatte, verrechnet, weil nur diese das Ergebnis des Geschäftsjahrs beeinflussen.

Diejenigen deutschen Aktiengesellschaften, die zwar keine Feuerversicherung betreiben, aber ihrem Hauptgeschäfte nach - abgesehen von der Transportversicherung doch zur Gruppe IV gehören (Fides, Fortuna, Neptun, Stuttgarter Mit- und Rückversicherungs - Aktien - Gesellschaft), sind unter die Feuerversicherungsaktiengesellschaften eingereiht worden, soda $\beta$ sich die Zahl der in Betracht zu ziehenden Gesellschaften auf 34 stellt. Davon scheidet eine (Providentia) aus, weil ihre Sachversicherung nicht von dem Lebensversicherungsgeschäfte getrennt werden konnte; die Betriebsrechnung dieser Gesellschaft findet sich bei Gruppe I (Tab. I 33). Die Gegenseitigkeitsvereine sind in der Gewinn- und Verlustrechnung vollständig vertreten, weil sie sich sämtlich auf die Feuerversicherung beschränken.

In der Gewinn- und Verlustrechnung der Aktiengesellschaften findet sich die Summe der Sonder-Einnahmen aller Zweige mit 116489146 , // verzeichnet, davon rühren $98627774 \mathbb{N}$ oder 84,7 Prozent aus der Feuerversicherung, $485 \mathbb{N}$. aus der Sturmschädenversicherung, $700324 \mathscr{N}$ oder 0,6 Prozent aus der Wasserleitungsschädenversicherung und 1 113632 $\mathscr{M}$ oder 1,5 Prozent aus der Einbruchdiebstahlversieherung, der Rest von 15446931 , th oder 13,3 Prozent aus Versicherungszweigen her, die zu anderen Gruppen gehören.

Die Summe der Sonder - Ausgaben aller Zweige 72056567 ell setzt sich zusammen aus 59255001 , $/ 1$ oder 82,2 Prozent aus der Feuerversicherung, 861 ctl aus der Sturmschädenversicherung, 311513 , Al oder 0,4 Prozent aus der Wasserleitungsschädenversicherung, 508907 , oder 0,i Prozent aus der Einbruchdiebstahlversicherung und 1198028 all oder 16,6 Prozent aus anderen Zweigen.

Aus der Gewinn- und Verlustrechnung ist $\mathrm{zu}$ entnehmen, daß betragen hat:

\begin{tabular}{|c|c|c|c|}
\hline & $\begin{array}{l}\text { bei der Gesanitheit } \\
\text { der Unternehmungen }\end{array}$ & $\begin{array}{l}\text { bei } 34 \text { Aktien- } \\
\text { gesellschaften }\end{array}$ & $\begin{array}{l}\text { bei } 16 \begin{array}{l}\text { Gegenscitigkeits- } \\
\text { vereinen }\end{array}\end{array}$ \\
\hline e Summe aller Einna & $156954320 A$ & $126132910 \mathrm{Al}$ & $30821410 \mathrm{dt}$. \\
\hline mme aller Ausg & $119887712 "$ & 106583896, & $13303816 \%$ \\
\hline r Jahresüberschuß & 37066608, & $19549014 "$ & I7517594, \\
\hline
\end{tabular}

Von den gesamten Einnahmen entfallen bei den Aktiengesellschaften auf die Sonder-Einnahmen 116489146 .th oder 92,4 Prozent, auf den Ertrag der Kapitalanlagen 8390338 At oder 6,7 Prozent, der Rest von 1253426 \% oder 0,9 Prozent auf andere Einnahmen; von den gesamten Ausgaben auf die Sonder-Ausgaben $72056567 \mathrm{~d}$ oder 67,6 Prozent, auf Verwaltungskosten und Steuern $32146960 \mathscr{M l}$ oder 30,2 Prozent, auf Abschreibungen 517901 th oder 0,5 Prozent und 1862468 . $/$ oder 1,7 Prozent auf die übrigen Ausgaben. Bei den Gegenseitigkeitsvereinen kommen von den gesamten Einnahmen $28+14894$ dl oder 92,2 Prozent auf die Sonder-Einnahmen, 2083788 all oder 6,8 Prozent auf die Kapitalerträge und 322728 \&ll oder 1,0 Prozent auf die anderen Einnahmen; von den gesamten Ausgaben $8620966 \mathscr{N l}$ oder 64,8 Prozent auf die Sonder-Ausgaben, $4323827 \mathscr{M}$ oder 32,s Prozent auf Verwaltungskosten und Steuern, 172666 Nl oder 1,3 Prozent auf Abschreibungen und $186357 \mathscr{N}$ oder 1,4 Prozent auf die übrigen Ausgaben. 
Von den Gegenseitigkeitsvereinen haben aus dem Geschäftsbetrieb des Jahres r 903 vier einen Verlust von zusammen 80269 , $/$, die übrigen 12 einen Überschuß von $17597863 . \%$ gehabt, wovon aber $14 \$ 664249$. /l allein auf die Gothaer Feuerversicherungsbank und 1686702 Nl auf die Württembergische Privatfeuerversicherung kommen. Diese Überschüsse sind kein Gewinn im gewöhnlichen Sinne, sie haben vielmehr in der Art der Prämienbemessung ihren Grund; schaltet man daher diese beiden Vereine aus, so bleibt für die übrigen 15 nach Abzug des Verlustes ein Gesamtgewinn von 1166643 .// oder 16,3 Prozent der Sonder-Einnahmen dieser Vereine. I)ie Aktiengesellschaften haben im Berichtsjahre sämtlich mit Gewinn gearbeitet, ihr Überschuß beziffert sich auf 16,8 Prozent der Sonder-Einnahmen. Der Gewinn der Aktiengesellschaften vermindert sich aber noch ganz beträchtlich, wenn man sich auf den eigentlichen Versicherungsbetrieb im Berichtsjahre beschränkt und den Gewinn aus der Vermögensverwaltung ausschaltet. Für den reinen Versicherungsbetrieb gewinnt man ein annähernd zutreffendes Bild, wenn man die Summe der Sonder-Einnahmen gegenüberstellt der Summe der Sonder-Ausgaben, vermehrt um die Verwaltungskosten und Steuern. Man findet dann, daß das Versicherungsgeschäft von fünf Gesellschaften einen Verlust von zusammen $402680 . / /$ gebracht hat, und daß sich der Gewinn der übrigen nur noch auf 1ํㅡㄴ 668299 dt stellt, der Betriebsgewinn aller 32 Aktiengesellschaften beläuft sich also für das Jahr 1903 auf nur $12285619 \mathscr{N}$ oder 10,5 Prozent der Sonder-Einnahmen nebst Abnahme der Prämienüberträge.

Zur Beurteilung des Geschäftsbetriebs der ausländischen Gesellschaften im Berichtsjahr ist die Gewinn- und Verlustrechnung für das gesamte in- und ausländische Geschäft möglichst nach denselben Grundsätzen dargestellt, die bei den deutschen Unternehmungen zur Anwendung gekommen sind. Die Sonder-Einnahmen und die Sonder-Ausgaben finden sich in den Tabellen $I V 20$ bis 22 und die Gewinn- und Veriustrechnungen in Tab. IV 23.

Eine Bilanz ist in dieser Statistik grundsätzlich nur für das Gesamtgeschäft einer Die Bilanz jeden Gesellschaft gegeben und bei derjenigen Gruppe aufgeführt, zu der das Unternehmen seinen Hauptbetriebe nach - die Transportversicherung ausgeschlossen gehört. Dem entsprechend ist die Bilanz der Providentia und die der Bayerischen Hypotheken- und Wechselbank bei Gruppe I behandelt, sodaß hier nur die Bilanzen für 32 deutsche Aktiengesellschaften und 16 Gegenseitigkeitsvereine darzustellen sind. Faßt man diese zusammen, so schlielßt die Bilanz für die Aktiengesellschaften mit $391524923 \mathscr{M}$ und für die Gegenseitigkeitsvereine mit 61194751 all ab. Im übrigen mögen folgende Zahlen daraus hier zusammengestellt werden.

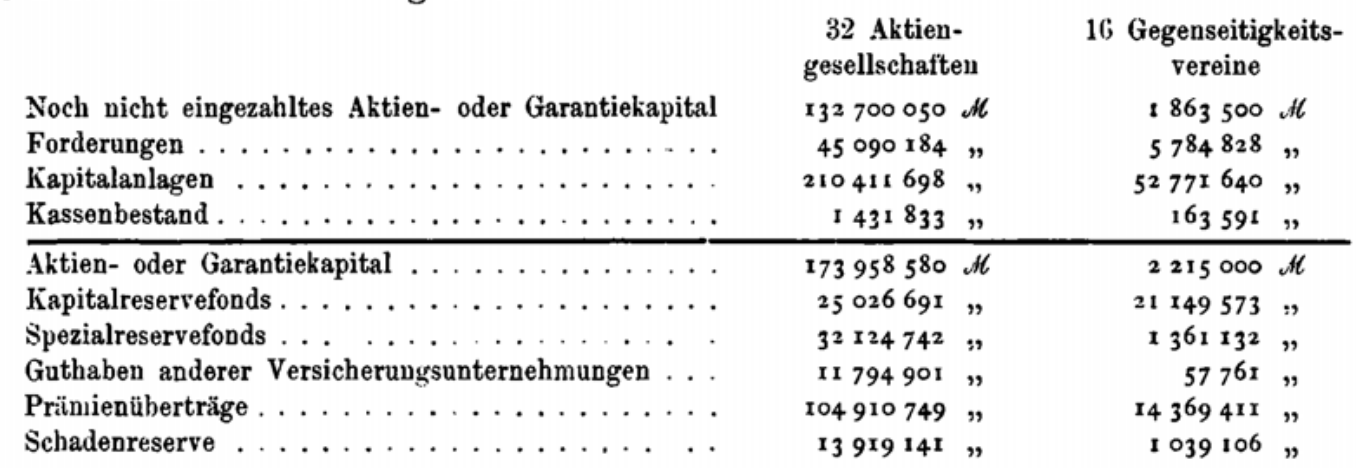

Bei den Aktiengesellschaften kamen hiernach von $1000 \mathscr{H}$ der Aktiva 339 Al auf noch nicht eingezahltes Aktienkapital, 115 all auf Forderungen, 537 dl auf Kapitalanlagen und 4 , /l auf Kassenbestand; von 1000 ، $/$ der Passiva 444 Nh auf Aktienkapital, $64 \mathrm{dl}$ auf Kapitalreserve, $82, / l$ auf Spezialreserven, $30 \mathrm{dl}$ auf Guthaben anderer Versicherungsunternehmungen, $268 \mathrm{dl}$ auf Prämienüberträge und $36 \mathrm{~d}$ auf Schadenreserve. 
Bei den Gegenseitigkeitsvereinen dagegen $30, / /$ auf noch nicht eingezahltes Garantiekapital, 95, $/$ auf Forderungen, $862,1 /$ auf Kapitalanlagen, $3, \|$ auf Kassenbestand: 36 . $/$ auf Garantiekapital, 346 , $/$ auf Kapitalreserve, 22 . $/$ auf Spezialreserven, 1 . 1 auf Guthaben anderer Versicherungsunternehmungen, 235, , auf Prämienüberträge und $17, \mathbb{A}$. auf Schadenreserve.

Die Kapitalanlagen (Tab. IV 25) verteilen sich auf folgende Wertarten:

\begin{tabular}{|c|c|c|c|c|c|c|c|c|}
\hline \multirow[b]{2}{*}{ Grundbesitz. . . . . . . . . . } & \multicolumn{4}{|c|}{$\begin{array}{l}\text { bei } 32 \text { Aktien- } \\
\text { gesellschaften }\end{array}$} & \multicolumn{4}{|c|}{$\begin{array}{c}\text { bei } 16 \text { Gegenseitigkeits- } \\
\text { vereinen }\end{array}$} \\
\hline & 17416626 & At & oder & $8,3 \%$ & 2389839 & dt & oder & $4,5 \%$ \\
\hline Hypotheken und Grundschulden . & 105232793 & $"$ & $"$ & 50,0, & 20989975 & $"$ & $"$ & 39,8, \\
\hline Wertpapiere .............. & 79216952 & $"$ & " & 37,6, & 27064446 & $"$ & $"$ & 51,3, \\
\hline Darlehen auf Wertpapiere......... & I 409800 & $"$ & " & 0,7, & - & $"$ & $"$ & $-n$ \\
\hline Wechsel $\ldots \ldots \ldots \ldots \ldots \ldots$ & 3150073 & $"$ & $"$ & $\mathbf{x}, 5$, & 56762 & $"$ & $"$ & o,r, \\
\hline Sonstige Anlagen $\ldots \ldots \ldots \ldots$ & 3985454 & 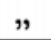 & $"$ & ז,9 & 2270618 & $"$ & $"$ & 4,3, \\
\hline
\end{tabular}

Vergleicht man die Kapitalanlagen derjenigen Unternehmungen, welche in beiden Statistiken behandelt worden sind, am Ende des Berichtsjahrs mit denjenigen am Ende des Vorjahrs, so findet man eine Zunahme um $891876 \mathscr{M l}$ bei dem Grundbesitz, um $12437854 \mathscr{M}$ bei den Hypotheken und Grundschulden und um $1401341 \mathcal{N}$ bei den Wertpapieren. Zurückgegangen sind die Darlehen auf Wertpapiere um 693651 , $M$, der Bestand an Wechseln um $412708 \mathscr{M}$. und die sonstigen Anlagen um $261196 \mathfrak{N}$.

Die Forderungen bestehen in:

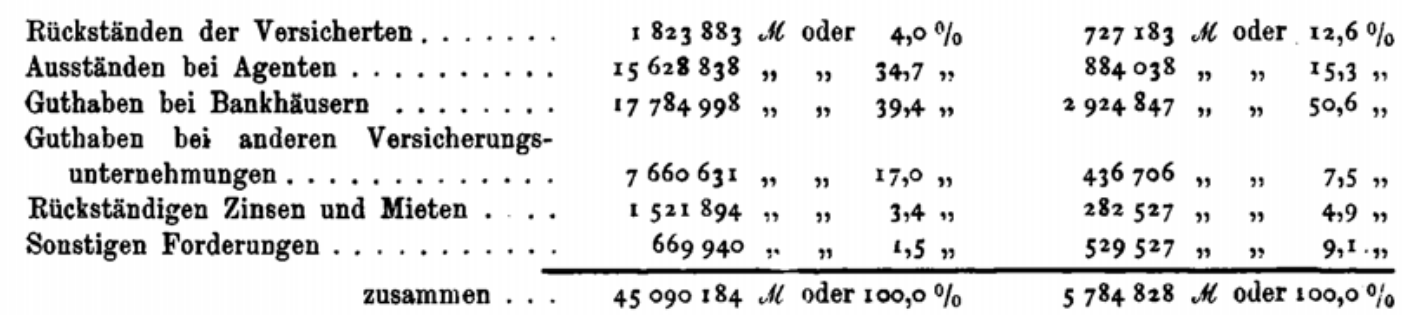

Die Bilanz weist für vier Gegenseitigkeitsvereine einen Verlust von zusammen $90021 \mathscr{M}$ nach; davon rühren $9752 \mathscr{N}$ aus dem Vorjahre her. Alle anderen Unternehmungen haben günstig abgeschlossen. Der bilanzmäßige Gewinn beträgt für die Aktiengesellschaften $19033713 \mathscr{A l}$ und für die Gegenseitigkeitsvereine $17692192 \mathscr{N}$, er ist nach Tab. IV 26 wie folgt verteilt worden:

\begin{tabular}{|c|c|c|c|c|c|c|c|}
\hline \multirow{4}{*}{$\begin{array}{l}\text { an den Kapitalreservefonds } \ldots \ldots \ldots \ldots \\
\text { an sonstige Reserven } \ldots \ldots \ldots \ldots \ldots\end{array}$} & \multicolumn{3}{|c|}{32 Aktiengesellschaften } & \multicolumn{4}{|c|}{16 Gegenseitigkeitsvereine } \\
\hline & $715 \circ 32$ & A oder & $3,8 \%$ & $55535^{8}$ & $\mathscr{M}$ & oder & $3,20: 0$ \\
\hline & 3442396 & $"$ & 18,1, & 196344 & $"$ & $\because$ & $\mathbf{I}, \mathbf{I}$, \\
\hline & 669891 & $"$ & $3,5 \%$ & 220000 & $"$ & $"$ & $\mathbf{1}, \mathbf{2}$, \\
\hline r Tantièmen ..... & 1470312 & " & 7,7, & 32455 & $"$ & $"$ & 0,2, \\
\hline die Aktionäre (Garanten) . . & 11135717 & $"$ & $58,5 \%$ & - & $"$ & $"$ & - \\
\hline die Versicherten ........... & - & & 一 & 16594598 & 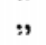 & $"$ & 93,8, \\
\hline lerweit. & 833211 & $"$ & $4,+n$ & $275^{8}$ & $"$ & $"$ & 0,02, \\
\hline rag auf neue Rechnu & $76 ; \times 54$ & 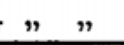 & 4,0, & 90679 & $"$ & $"$ & 0,5, \\
\hline
\end{tabular}

Die Kapitalreservefonds aller Unternehmungen stehen in der diesjährigen Bilanz höher als in der vorjährigen bei den Aktiengesellschaften um $803715 \mathbb{N}$, bei den Gegenseitigkeitsvereinen um 715977 cAf: die Spezialreserven stehen jetzt höher bei den Aktiengesellschaften um 3619006 AH und bei den Gegenseitigkeitsvereinen um 110486 ، $N$. 
Für die ausländischen Gesellschaften ist die Bilanz der Assicurazioni Generali bereits bei der Lebensversicherung in Tab. I ja und die der Schweizerischen UnfallversicherungsGesellschaft und der Zürich bei der Unfall- und Hapftpflichtversicherung in Tab. 1117 gegeben, die Bilanzen der übrigen 26 Unternebmungen finden sich in Tab. $1 V^{2} 29$. Im Anschlusse daran sind in Tab. IV 30 die Kapitalanlagen und die Aktivforderungen erläutert. Wegen des großen Interesses, das der Art der Kapitalanlagen zukommt, sind in der folgenden Übersicht die Gesellschaften nach Heimatländern zusammengefaßt und zum Vergleiche die Verhältniszahlen für die deutschen Unternehmungen wiederholt.

Ion je 1000 , / Kapitalanlagen entfielen auf:

\begin{tabular}{|c|c|c|c|c|c|c|c|}
\hline bei & $\begin{array}{l}\text { Grund- } \\
\text { besitz }\end{array}$ & $\begin{array}{c}\text { Hypotheken } \\
\text { und } \\
\text { Grund- } \\
\text { schulden }\end{array}$ & $\begin{array}{l}\text { Wert- } \\
\text { papiere }\end{array}$ & $\begin{array}{l}\text { Darlehen } \\
\text { auf } \\
\text { Wert- } \\
\text { papiere }\end{array}$ & Wechsel & $\begin{array}{l}\text { Sonstige } \\
\text { Anlagen }\end{array}$ & $\begin{array}{c}\text { Gesamtbetrag } \\
\text { der } \\
\text { Kapitalanlagen }\end{array}$ \\
\hline 2 belgischen Gesellschaften & 45 & - & 947 & 7 & I & - & $8379789 \mu$ \\
\hline 1 dänischen Gesellschaft $\ldots \ldots \ldots$ & 160 & 651 & 189 & - & - & - & $2054817 \%$ \\
\hline 13 englischen Gesellschaften . . . . . . & 129 & 136 & 654 & 37 & 3 & 41 & I $243806229 "$ \\
\hline 1 französischen Gesellschaft . . . . . & 148 & - & 852 & - & $\cdot$ & - & $19: 60768 \%$ \\
\hline 2 niederländischen Gesellschaíten . . . . & 141 & 97 & 487 & 275 & - & - & 6121803 \\
\hline . österreichischen Gesellschaften . . . . & 83 & - & 873 & 2 & 42 & - & $13546818 \%$ \\
\hline 2 sch wedischen Gesellschaften . . . . . & 63 & +90 & 282 & 38 & - & 127 & $50495567 "$ \\
\hline $\begin{array}{c}2 \text { schweizerischen Gesellschaften } \ldots \ldots \\
\text { dagegen bei }\end{array}$ & 103 & 257 & $5^{82}$ & - & $5^{8}$ & - & $16899400 "$ \\
\hline 32 deutschen Aktiengesellschaften .... & 83 & 500 & 376 & 7 & 15 & 19 & 210411698 \\
\hline 16 deutschøn Gegenseitigkeitsvereinen. . & 45 & 398 & 513 & - & $\mathbf{r}$ & 43 & $52771640 "$ \\
\hline
\end{tabular}

Für die im Eingange dieses Absehnitts erwähnten 11 kleinen Feuerversicherungsunternehmungen sollen hier zur Ergänzung einige Zahlen angeführt werden, wobei aber Unternenausdrücklich betont werden muß, daß diese Zahlen keineswegs ein Urteil über die Be- mungen deutung der kleinen Vereine im Deutschen Reiche zulassen. Die Zahl der überhaupt vorhandenen ist sehr viel größer, und es werden sich unter der Aufsicht der Landesbehörden auch gewiß mehrere finden, welche die unter Reichsaufsicht stehenden kleinen Vereine an Umfang übertreffen. Hier kommen insgesamt 53426 Versicherungen mit rund 531,66 Millionen Mark in Frage; der durchschnittliche Betrag einer Versicherung stellt sich danach auf $9951 \%$. Die gesamten Einnahmen haben im Jahre 1903885067 \% betragen, davon kommen 832711 ، $/$ oder 94,1 Prozent auf Prämien, 3247 Al oder 0,4 Prozent auf Nebenleistungen der Versicherten und $18579 \mathscr{M}$ oder 2,1 Prozent auf Kapitalerträge; von den gesamten Ausgaben in Höhe von 820894 $/$ kommen 574143 69,9 Prozent auf Entschädigungen und 53890 , oder 5,6 Prozent auf Verwaltungskosten. Als Vermögen am Schlusse des Berichtsjahrs haben die 11 Vereine zusammen $736439 \mathcal{M l}$. nachgewiesen.

\section{Gruppe V, Sonstige verschiedene Versicherungszweige.}

\section{Glasversicherung.}

Auf dem Gebiete der Glasversicherung haben im Jahre 1903 unter Reichsaufsicht Die Unter19 deutsche Aktiengesellschaften, 4 deutsche Gegenseitigkeitsvereine und 5 ausländische ${ }^{\text {nebmungen }}$ Gesellschaften gearbeitet; zu den vorjährigen Gesellschaften ist die Stuttgarter Mit- und Rückversicherungs - Aktien - Gesellschaft hinzugekommen. Davon beschäftigten sich 5 Aktiengesellschaften, 3 Gegenseitigkeitsvereine und 3 ausländische Gesellschaften ausschließlich mit diesem Versicherungszweige; für die übrigen Unternehmungen bildet die Glasversicherung nur einen Nebenbetrieb. 
LXVIII

Gruppe $\mathbf{V}$

Umfang Über den Umfang des Geschäfts der deutschen Unternehmungen in der Glasversichedes Ge- rung soll Tab. V 1 unterrichten. Da die Versicherungssumme bei zwei Gesellschaften deutsoben nicht angegeben werden kann, so empfiehlt sich hier die Beschränkung auf diejenigen

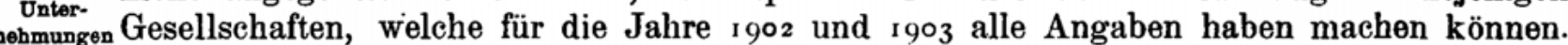
Bei der Besprechung der Prämien und der Schäden im Anschluß an Tab. V 5 wird Gelegenheit sein, auf den Geschäftsumfang aller Gesellschaften im Jahre r903 zurückzukommen.

An selbst abgeschlossenen Versicherungen liefen einschließlich der in Rückdeckung gegebenen Beträge:

Ende $1902 \quad$ Ende 1903

bei 17 Aktiengesellschaften ..... 327716 Vers. über $x 60 \times 29678 d \mathcal{A}, 348294$ Vers. über 171352872 h

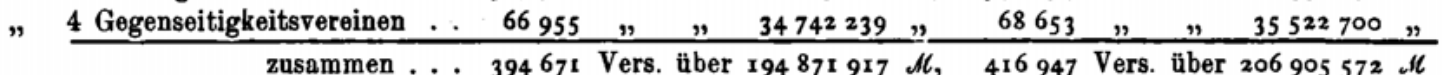

Demnach hat die Zahl der Versicherungen bei den Aktiengesellschaften um 20 อ̃78, bei den Gegenseitigkeitsvereinen um 1 698, bei allen Unternehmungen zusammen um 22276 zugenommen; die Versicherungssumme bei den Aktiengesellschaften um $11223194 \mathscr{N}$, bei den Gegenseitigkeitsvereinen um $810461 \mathrm{~N}$; insgesamt ist sie also um 12033655 gestiegen.

Der im Durchschnitt auf eine Versicherung entfallende Betrag belief sich bei den Aktiengesellschaften Ende r 902 auf $489 \mathscr{M}$ und Ende 1903 auf $492 \mathscr{H}$, bei den Gegenseitigkeitsvereinen auf 519 und $518 \mathscr{N}$, bei allen Unternehmungen auf 494 und $496 \mathscr{N}$. Außerdem hatten die Aktiengesellschaften noch Versicherungen in Höhe von 449887 A am Schlusse des Jahres 1902 und in Höhe von $394908 \mathscr{M}$ am Schlusse von 1903 in Rückdeckung übernommen, die Gegenseitigkeitsvereine solche in Höhe von $8925 \mathscr{M}$ und $4568 \mathscr{M}$, sodaß der gesamte Betrag der Versicherungen bei den Aktiengesellschaften Ende 1902 $160579565 \mathscr{M}$. und Ende r $9 \circ 3171747780 \mathscr{H}$, bei den Gegenseitigkeitsvereinen $34751164 \mathscr{M}$ und $35557268 \mathscr{A}$, bei allen Unternehmungen zusammen $195330729 \mathscr{N}$ und $207305048 \mathscr{H}$ betrug. Der Anteil des mittelbaren Geschäfts am Gesamtgeschäft ist danach ganz unbedeutend gewesen, er stellte sich bei den Aktiengesellschaften im Jahre 1902 auf 0,3 und im Jahre 1903 auf 0,2 Prozent, und war bei den Gegenseitigkeitsvereinen noch kleiner.

Die Tabellen V 2 und 3 zeigen, in welchem Umfange bei den deutschen Unternehmungen das inländische und ausländische Glasversicherungsgeschäft an dem in Tab. V 1 dargestellten Gesamtgeschäfte beteiligt ist.

Von den selbst abgeschlossenen Versicherungen derjenigen Unternehmungen, bei denen die Zahl der Versicherungen und die Versicherungssummen angegeben sind, kommen:

$$
\text { Ende } 1902 \quad \text { Ende } 1903
$$

bei 17 Aktiengesellschaften

auf das inländische Geschäft . . 309581 Vers. über ${ }_{151} 949678 \mathscr{M}, \quad 328724$ Vers. über $162251056 \mathscr{H}$

.auf das ausländische Geschäft. . $18135, " 8180000, \quad$ " $19570 \quad$ " 9101816,

bei 4 Gegenseitigkeitsvereinen

auf das inländische Geschäft . . 58042 Vers. über $28659796 \mathrm{Al}, \quad 59698$ Vers. über 29351539 At

auf das ausländische Geschäft. . 89 I $_{3}$ " " 6082443 " 8955 " "6201 161 "

Das inländische direkte Geschäft hat also bei den 17 Aktiengesellschaften um 19143 Versicherungen und 10301378 oth und bei 4 Gegenseitigkeitsvereinen um 1656 Versicherungen und $691743 \mathscr{M}$. zugenommen; das ausländische direkte Geschäft dagegen bei den Aktiengesellschaften um 1514 Versicherungen und 921816 ، $\%$, bei den Gegenseitigkeitsvereinen um 42 Versicherungen und $118718 \mathscr{M}$.

Der durchschnittlich auf eine selbst abgeschlossene Versicherung entfallende Betrag ist bei dem deutschen Geschäfte der Aktiengesellschaften von 491 auf 494 , // gestiegen, bei dem der Gegenseitigkeitsvereine von 494 auf 492 , gefallen. Bei dem ausländischen 
Geschäfte war die Änderung etwas größer, bei den Aktiengesellschaften ist or von 451 auf $465, \mathscr{N}$ und bei den Gegenseitigkeitsvereinen von 682 auf $692 \mathscr{M}$. angewachsen.

Die fraglichen Aktiengesellschaften hatten Ende 1902 noch 32473 \& . im inländischen und $417414 \mathscr{M}$ im ausländischen Geschäft in Rückdeckung übernommen, Ende r 903 31679 . $/$ im inländischen und 363229 , $\%$ im ausländischen Geschäfte. Die Gegenseitigkeitsvereine haben im Auslande keine mittelbaren Geschäfte gemacht, aus dem Inlande hatten sie $8925, \%$ am Ende des Jahres 1902 und nur $4568, \|$ am Ende des Jahres r 903 in Rückdeckung übernommen.

Zur weiteren Erläuterung des Verhältnisses des inländischen und des ausländischen Geschäfts zum Gesamtgeschäfte wird folgende Zusammenstellung dienen.

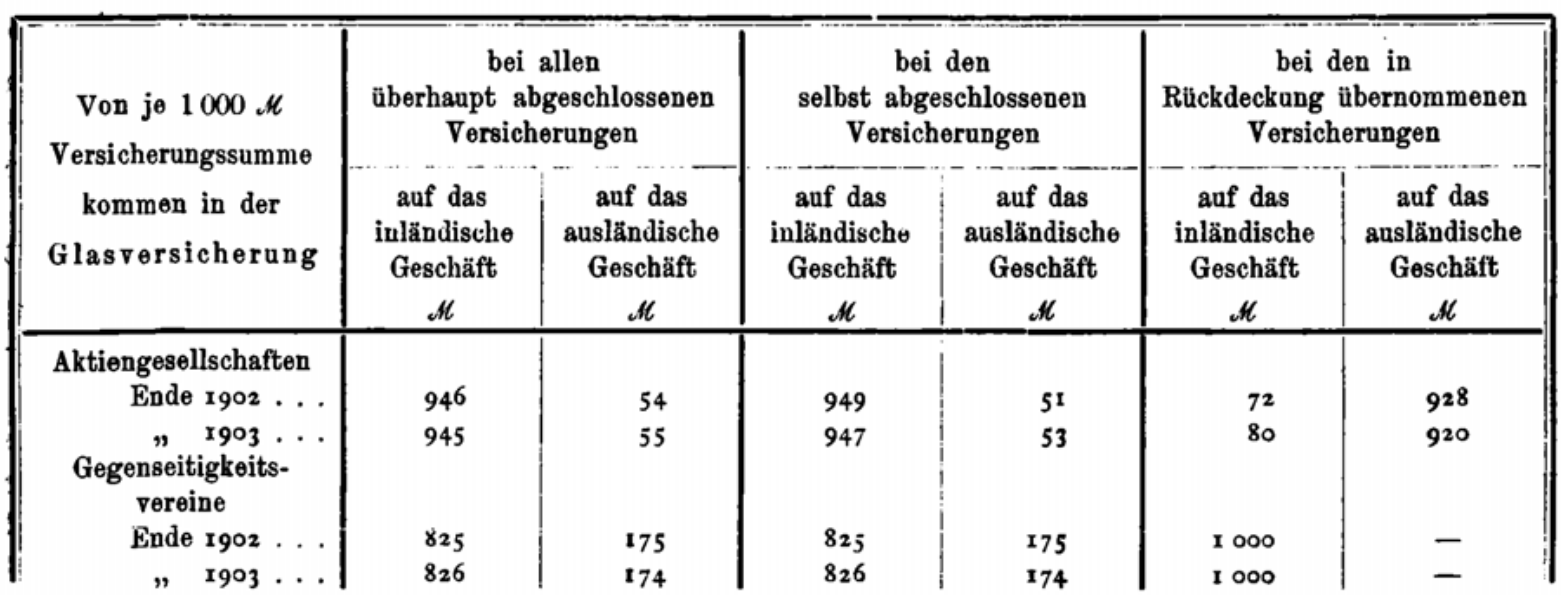

Der Umfang des deutschen Geschäfts der ausländischen Gesellschaften ist nur klein Umfaug (Tabelle V 4), es liegt ganz überwiegend in der Hand einer Gesellschaft, die Ende $1902 \underset{\text { deutschen }}{\text { des }}$ 8067 und Ende 19038279 Versicherungen laufen hatte, bei der aber für das Ende des Geschäts Berichtsjahrs die Versicherungssumme nicht angegeben werden kann. Bei den andern vier der ausGesellschaften waren Ende r902 2383 Versicherungen über 1174797 all und Ende r903 Unter2626 Versicherungen über $1238336 \mathscr{M}$ in Kraft; der durchschnittlich auf eine Versicherung ${ }^{\text {nehmungen }}$ kommende Betrag belief sich am Ende des Vorjahrs auf 493 ، $N$, am Ende des Berichtsjahrs auf 472 . $\%$. Das Geschäft ist von allen Gesellschaften nur unmittelbar betrieben worden.

Die Bruttoprämieneinnnahme im Geschäftsjahr und die im Geschäftsjahr entstandenen Die BruttoBruttoschäden sind für die deutschen Unternehmungen in Tabelle V 5 und für das deutsche $\begin{gathered}\text { pramien } \\ \text { und }\end{gathered}$ Geschäft der ausländischen Gesellschaften in Tabelle V 6 zusammengestellt; dabei können schăden alle Gesellschaften in Betracht gezogen werden.

In der Glasversicherung haben im Jahre 1903 die 19 deutschen Aktiengesellschaften 4332392 . $/$ und die 4 Gegenseitigkeitsvereine 841694 « , alle Unternehmungen zusammen also $5174086 \mathscr{A l}$ an Prämien eingenommen, und es sind in diesem Jahre den Aktiengesell-. schaften Schäden im Betrage von $2376531 \mathrm{~d}$, den Gegenseitigkeitsvereinen solche im Betrage von 485066 . /l, zusammen im Betrage von 2861597 , $/$ entstanden. Diese Schäden können aber nicht zu den Prämien in Beziehung gebracht werden, weil die Prämien nicht die Prämien für das Geschäftsjahr, sondern die im Geschäftsjahr überhaupt eingenommenen Beträge sind und mithin über das Berichtsjahr vielfach hinausreichen, wogegen die in früheren Jahren für im Jahre 1903 noch laufende Versicherungen gezahlten Prämien fehlen; aus demselben Grunde können die Prämien auch nicht zu den durchschnittlichen Versicherungssummen in Beziehung gebracht werden. 
Bei den Aktiengesellschaften kommen von der Prämieneinnahme 94,2 Prozent auf das inländische und 5,8 Prozent auf das ausländische Geschäft; die inländischen Prämien kommen so gut wie ganz auf selbst abgeschlossene Versicherungen, von den ausländischen Prämien fallen dagegen 96,0 Prozent auf das unmittelbare und 4,0 Prozent auf das mittelbare Geschäft. Von den überhaupt entstandenen Schadensummen der Aktiengesellschaften kommen 93,7 Prozent auf den inländischen und 6,3 Prozent auf den ausländischen Betrieb; die inländischen Schäden stammten bis auf den geringen Betrag von $1818 \%$, die ausländischen zu 94,4 Prozent aus selbst abgeschlossenen Versicherungen.

Der durchnittliche Betrag eines Schadens stellt sich für die Aktiengesellschaften im inländischen Betriebe beim unmittelbaren Geschäft auf $39, / 1$, beim mittelbaren auf 114 ، , im ausländischen Betrieb auf $38 \mathfrak{N}$ und auf $8 \mathrm{~d}$.

Bei den vier Gegenseitigkeitsvereinen kommen von der Prämieneinnahme 83,2 Prozent auf das inländische und 16,8 Prozent auf das ausländische Geschäft; von den inländischen Prämien kommt ein verschwindender Bruchteil auf die in Rückdeckung übernommenen Versicherungen, während die ausländischen vollständig aus dem direkten Geschäfte stammen. Die entstandenen Schäden rühren dementsprechend auch fast vollständig aus den unmittelbaren Abschlüssen her, auf den inländischen Betrieb kommen 86,0 Prozent der Schadenzahlungen, auf den ausländischen 14,0 Prozent.

Der durchschnittliche Betrag eines Schadens stellt sich für die deutschen Gegenseitigkeitsvereine im Inland auf $38 \mathcal{H}$, im Ausland auf $17 \mathscr{M}$.

Die fünf ausländischen Gesellschaften haben im Jahre 1903 aus dem deutschen Glasversicherungsgeschäfte (Tabelle V 6) insgesamt 159276 / Bruttoprämien eingenommen, für Schäden hatten sie $148643 \mathscr{N}$, oder durchschnittlich für einen Schadenfall $84 \mathfrak{A}$. bereit zu stellen.

Die Rech- Die meisten Gesellschaften, die auf dem Gebiete der Glasversicherung arbeiten, nungs- betreiben diese Versicherungsart als Nebengeschäft neben anderen Versicherungsgeschäften. ge- Dabei halten sie die verschiedenen Zweige nicht immer vollständig getrennt, sondern verrechnen gewisse Posten, namentlich den Gewinnvortrag und die Kapitalerträge einerseits und die Verwaltungskosten, Steuern, Abschreibungen und Verluste aus der Vermögensverwaltung andererseits, nur für das Gesamtgeschäft. Es sind deshalb, wie in den vorhergehenden Abschnitten für die anderen Versicherungszweige, so hier für die Glasversicherung, die Sonder-Einnahmen und Sonder-Ausgaben dieses Zweiges für sich berechnet (Tabelle V7), und es ist für jede Unternehmung nur eine Gewinn- und Verlustrechnung für das Gesamtgeschäft aufgestellt und bei derjenigen Gruppe nachgewiesen worden, zu der die Gesellschaft ihrem Hauptbetriebe nach - die Transportversicherung ausgeschlossen - gehört. Der Gleichförmigkeit wegen ist bei denjenigen Unternehmungen, die allein in der Glasversicherung tätig sind, ebenso verfahren worden.

Als Sonder-Einnahmen sind hier wie bei Gruppe IV die Prämien für eigene Rechnung, und Aus- die Nebenleistungen der Versicherten, die Ersparnis. aus der Schadenreserve des Vorjahrs gaben des und die Abnahme der Prämienüberträge, als Sonder-Ausgaben die Schäden des GeschäftsBerichts-.
jahrs jahrs (nach Abzug des Anteils der Rückversicherer, des Erlöses für Bruchglas und der Schadenerstattungen), der Zuschuß zur Schadenreserve und die Zunahme der Prämienüberträge zusammengefaßt.

Bei den deutschen Unternehmungen betrug in der Glasversicherung

\begin{tabular}{|c|c|c|c|}
\hline & $\begin{array}{l}\text { bei der Gesamtheit } \\
\text { der Unternehmungen }\end{array}$ & $\begin{array}{l}\text { bei } 19 \text { Aktien- } \\
\text { gesellschaften }\end{array}$ & $\begin{array}{c}\text { bei } 4 \text { Gegenseitigkeits } \\
\text { vereinen }\end{array}$ \\
\hline en & $5145172 \lll$ & 4269945 d & $875227 \mu$ \\
\hline Summe der Sonder-Ausgaben . . & $3111095::$ & $2579910 "$ & $531185 \%$ \\
\hline
\end{tabular}


Von je 1000 , /l der Sonder-Einnahmen kamen

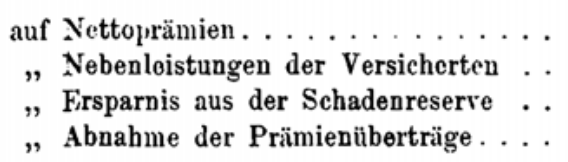

\begin{tabular}{|c|c|c|c|c|}
\hline $97 \mathrm{I}$ & " & $973 \cdot \mathscr{H}$ & 962 & $\mu$ \\
\hline 22 & $\because$ & $20 "$ & 30 & " \\
\hline 7 & $\eta$ & $7 \%$ & 8 & $"$ \\
\hline 0,1 & $"$ & $0,1 \quad$, & - & $"$ \\
\hline
\end{tabular}

Von 1000 .tl Sonder-Ausgaben kamen

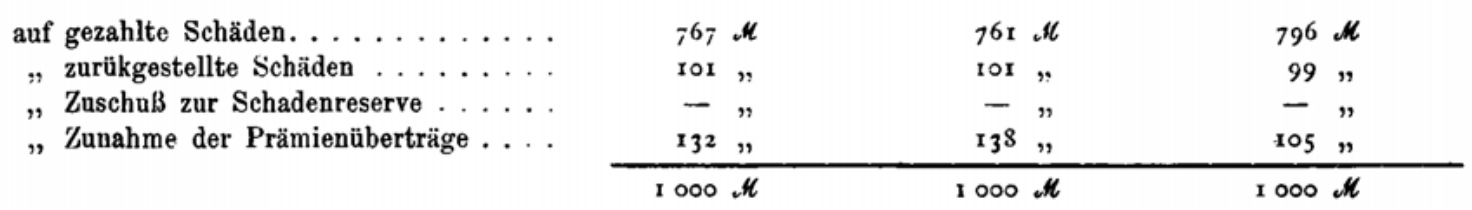

Von den in Geschäftsjahr entstandenen Schadensummen wurden durchschnittlich 88,4 Prozent noch im Geschäftsjahre gezahlt, bei den Aktiengesellschaften 88,3 Prozent, bei den Gegenseitigkeitsvereinen 88,9 Prozent; der Rest wurde der Schadenreserve überwicsen.

Die Gewinn- und Verlustrechnung soll nur das Ergebnis des Geschäftsjahrs darstellen, deshalb ist der Gewinnvortrag aus dem Vorjahre fortgelassen, und es sind hier, Betriebswie auch schon bei der Schadenreserve in Tabelle V 7, die Rücklagen nicht mit ihrem ganzen Betrag am Anfange des Jahres in Einnahme und am Schlusse des Jahres in Ausgabe nachgewiesen, sondern es sind nur ihre Änderungen, die der Geschäftsbetrieb des Jahres zur Folge hatte, verrechnet.

Die Gewinn- und Verlustrechnung ist in Tabelle V 8 nur noch für 6 Aktiengesellschaften und für 3 Gegenseitigkeitsvereine zu geben, für die übrigen Unternehmungen ist sie bereits bei den vorhergehenden Gruppen mitgeteilt.

Die Summe der Sonder-Einnahmen der 6 Aktiengesellschaften beträgt 4608286 , /l, davon stammen $2501127, \mathbb{N}$ aus der Glasversicherung und 2107159 , $/$. aus der Transportversicherung (allein des Deutschen Lloyd), die Summe der Sonder-Ausgaben boträgt $3084095 \mathrm{Al}$, wovon $1592792, / /$ aus der Glasversicherung und $1491303 \mathscr{N}$ aus der Transportversicherung herrühren.

Bei den 3 Gegenseitigkeitsvereinen kommt nur die Glasversicherung in Frage, die Summe der Sonder-Einnahmen stellt sich auf 870939 dl, die der Sonder-Ausgaben auf 527896 «t.

Aus der Gewinn- und Verlustrechnung ist zu entnehmen, daß betragen haben:

\begin{tabular}{|c|c|c|c|}
\hline & $\begin{array}{l}\text { bei der Gesamtheit } \\
\text { der Unternehmungen }\end{array}$ & $\begin{array}{l}\text { bei } 6 \text { Aktien- } \\
\text { gesellschaften }\end{array}$ & $\begin{array}{c}\text { bei } 3 \text { Gegenseitigkeits- } \\
\text { vereinen }\end{array}$ \\
\hline die Summe aller Einnahmen $\ldots \ldots$ & $5735064 M$ & $4820715 \cdot N$ & 914349 all \\
\hline die Summe aller Ausgaben ... & 5079427 & $4249 \times 73$ & $830254 "$ \\
\hline der Jahresüberschuß & 655637, & $571542 \#$ & $84095 n$ \\
\hline
\end{tabular}

Von den gesamten Einnahmen entfallen bei den 6 Aktiengesellschaften auf die Sonder-Einnahmen 4608286 , / oder 95,6 Prozent, auf den Ertrag der Kapitalanlagen 212392 .ll oder 4,4 Prozent, von den gesamten Ausgaben auf die Sonder-Ausgaben 3084095 ./l oder 72,6 Prozent, auf Verwaltungskosten und Steuern. $1110916 \mathrm{AH}$ oder 26,1 Prozent, auf Abschreibungen $9572 \mathscr{N}$ oder 0,2 Prozent und $4459 \mathscr{N}$. oder 1,1 Prozent auf sonstige Ausgaben.

Bei den 3 Gegenseitigkeitsvereinen kommen von den gesamten Einnahmen 870939 Al oder 95,3 Prozent auf die Sonder-Einnahmen, 38567 Ml oder 4,2 Prozent auf die Kapitalerträge und 4843 , // oder 0,5 Prozent auf sonstige Einnahmen; von den gesamten Ausgaben 527896 , th oder 63,6 Prozent auf die SonderAusgaben, 292672 ath oder 35,3 Prozent auf Ver- 
waltungskosten und Steuern, $4437, / /$ oder 0,5 Prozent auf Abschreibungen und 5249 ، oder 0,6 Prozent auf sonstige Ausgaben.

Alle Unternehmungen haben in dem Berichtsjahr einen Überschuß erzielt, mit Verlust hat keine von ihnen gearbeitet. Der Jahresüberschuß selbst ohne Gewinnvortrag. stellt sich bei den 3 Gegenseitigkeitsvereinen auf 9,7 Prozent, bei den 6 Aktiengesellschaften auf 12,4 Prozent der Sonder-Einnahmen.

Bei der geringen Bedeutung, welche den ausländischen Gesellschaften im Deutschen Reiche auf dem Gebiete der Glasversicherung zur Zeit zukommt, ist darauf verzichtet worden, ihre Betriebsrechnung und ihre Bilanz mitzuteilen.

Die Bilanz Die Bilanz der deutschen Unternehmungen ist in Tabelle V 9 für dieselben 6 Aktiengesellschaften und 3 Gegenseitigkeitsvereine gegeben, für welche auch die Gewinn- und Verlustrechnung mitgeteilt ist. Sie schließt für Ende ${ }_{1903}$ ab mit $10941521 \mathscr{M}$ bei den Aktiengesellschaften und mit 1207331 , t/ bei den Gegenseitigkeitsvereinen. Im übrigen mögen hier folgende Zahlen daraus zusammengestellt werden:

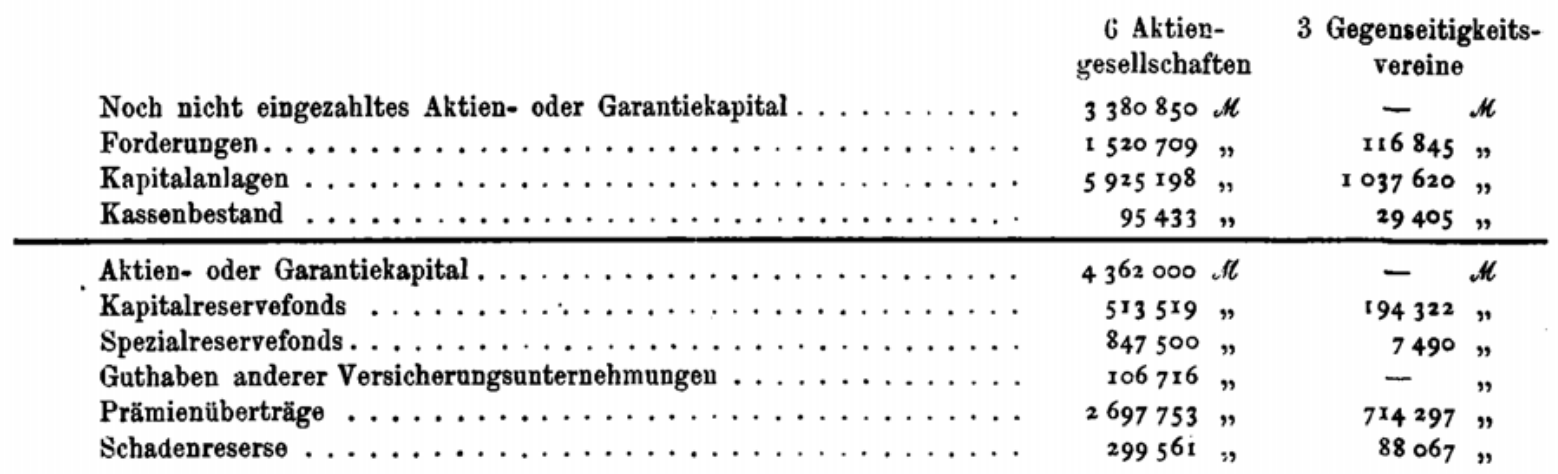

Bei den 6 Aktiengesellschaften kommen hiernach von 1000 / der Aktiva 309 dl. auf noch nicht eingezahltes Aktienkapital, 139 , ll auf Forderungen, $542 \mathcal{M}$ auf Kapitalanlagen und $9 \mathbb{N}$ auf Kassenbestand; von $1000 \mathrm{~N}$ der Passiva kommen $399 \mathscr{M}$ auf Aktienkapital, $47 \mathrm{~N}$ auf Kapitalreserve, $77 \mathrm{~N}$ auf Spezialreserven, $10 \mathscr{N}$ auf Guthaben anderer Versicherungsunternehmungen, $247 \mathrm{~N}$ auf Prämienüberträge und $27 \mathrm{cH}$ auf Schadenreserven. Bei den 3 Gegenseitigkeitsvereinen sind Garantiekapitale nicht vorhanden, im übrigen entfallen von 1000 , $/$ der Aktiva 97 . 1 . auf Forderungen, $859 \mathscr{H}$ auf Kapitalanlagen und 24 , $/$ auf Kassenbestand; von 1000 dt der Passiva entfallen 161 火. auf Kapitalreserve, $6 \%$ auf Spezialreserven, $592, \%$ auf Prämienüberträge und $73 \mathscr{M}$ auf Schadenreserve.

Die Kapitalanlagen (Tabelle V 10) verteilen sich auf folgende Wertarten:

\begin{tabular}{|c|c|c|c|c|c|c|c|}
\hline \multirow{6}{*}{$\begin{array}{l}\text { Grundbesitz } \ldots \ldots \ldots \ldots \\
\text { Hypotheken und Grundschulden } \ldots \ldots \\
\text { Wertpapiere. . . . . . . . . } \ldots \ldots \ldots \\
\text { Darlehen auf Wertpapiere } \ldots \ldots \ldots \ldots \\
\text { Wechsel } \ldots \ldots \ldots \ldots \ldots \ldots \ldots \ldots\end{array}$} & \multicolumn{3}{|c|}{$\begin{array}{l}\text { bei } 6 \text { Altien- } \\
\text { gesellschaften }\end{array}$} & \multicolumn{4}{|c|}{$\begin{array}{c}\text { bei } 3 \text { Gegenseitigkeits- } \\
\text { vereinen }\end{array}$} \\
\hline & I 293370 & $\mathscr{M}$ oder & $2 x, 8 \%$ & 46000 & 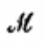 & oder & $4,4 \%$ \\
\hline & 4019130 & $"$ & 67,8, & 710600 & $"$ & ” & $68,5 \%$ \\
\hline & $5^{89} 151$ & $"$ & ro,o, & 281020 & $"$ & $"$ & 27,1, \\
\hline & 21008 & $"$ & $\circ, 4$, & - & $"$ & $"$ & $-n$ \\
\hline & 2539 & $"$ & ०,04 " & - & $"$ & $"$ & $-n$ \\
\hline
\end{tabular}

Die Kapitalanlagen sämtlicher 9 Unternehmungen haben Ende r 903 insgesamt $604114 \pi$ mehr betragen als am Schlusse des Vorjahrs, davon kamen $39370 \%$ auf Grundbesitz, $521880 \mathscr{N}$ auf Hypotheken, $30432 \mathrm{dl}$ auf Wertpapiere und $13145 \mathscr{M}$ auf Darlehen auf Wertpapiere, der Bestand an Wechseln ist um 713 dl zurückgegangen. 
Die Forderungen bestehen in:

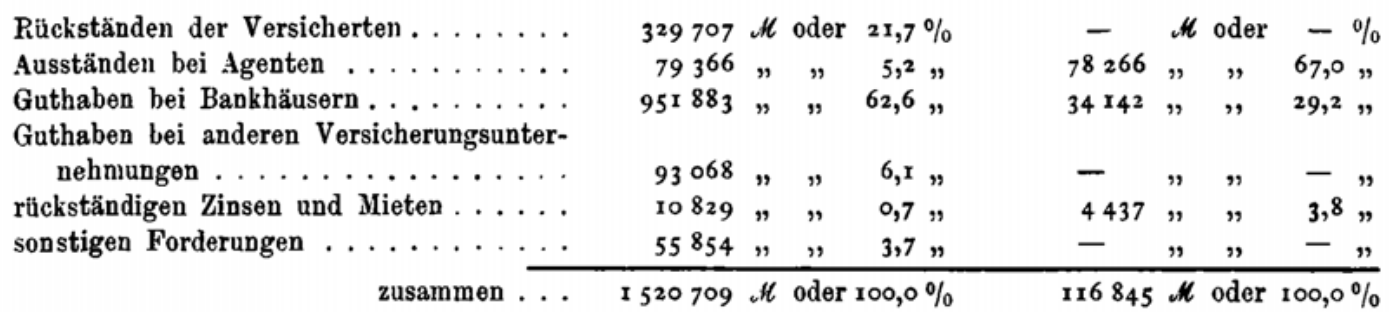

Der bilanzmäßige Gewinn ist nach Tab. V 11, wie folgt, verteilt worden:

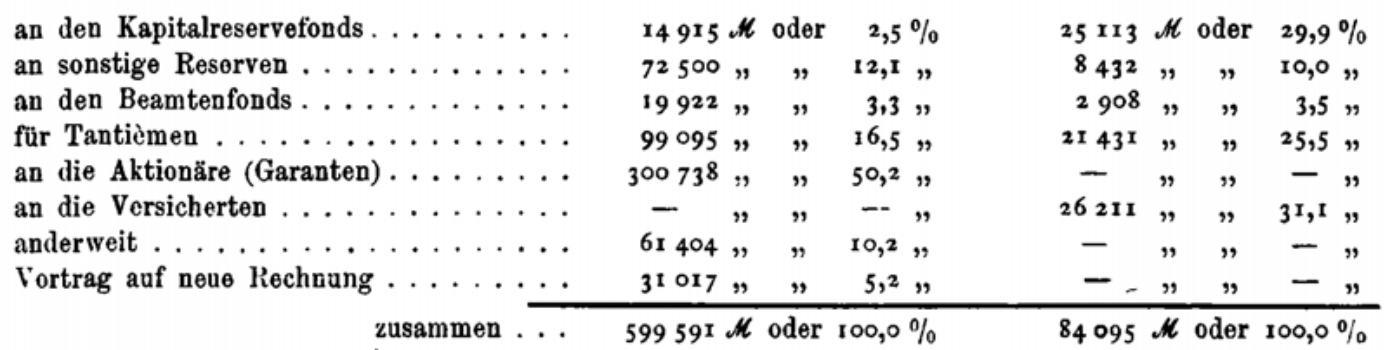

Der Kapitalreservefonds steht in der diesjährigen Bilanz höher als in der vorjährigen: bei den Aktiengesellchaften um $15336 \mathfrak{N l}$, bei den Gegenseitigkeitsvereinen um $21909 \mathscr{N}$. Die Spezialreserven sind bei den Aktiengesellschaften um $70161 \mathrm{~d} /$, bei den Gegenseitigkeitsvereinen um 2598 ell gewachsen.

\section{Kautions- und Garantieversicherung. '}

Dieser Versicherungszweig ist im Jahre 1903 nur nebenher betrieben worden; mit Geschäften von nennenswertem Umfange kommen dieselben beiden deutschen Gesellschaften wie in Vorjahr in Frage. Auf das kleine Geschäft, das der Atlas in Ludwigshafen und die Wilhelma in Magdeburg auf dem Gebiete der Kautionsversicherung erledigt haben, braucht hier nicht eingegangen zu werden (vgl. die Anmerkungen 4 und $5 \mathrm{zu}$ Tab. I 34 S. 109). Von ausländischen Gesellschaften waren zum Betriebe dieses Zweiges im Deutschen Reiche befugt, die Schweizerische Unfall-Versicherungs-Aktien-Gesellschaft in Winterthur und die Zürich, ihr deutsches Geschäft ist ebenfalls nur wenig umfangreich.

Über den Umfang des Geschäfts und seine Zusammensetzung aus inländischen und ausländischen sowie aus unmittelbaren und mittelbaren Abschlüssen gibt Tab. V 14 Aufschluß. Danach liefen bei den beiden deutschen Gesellschaften insgesamt an selbst abgeschlossenen Versicherungen einschließlich der in Rückdeckung gegebenen Beträge

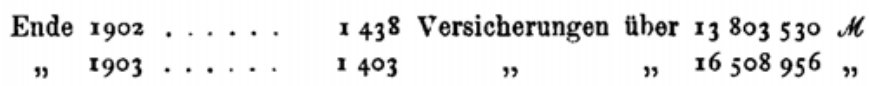

sodaß die Zahl der Versicherungen um 3ŏ abgenommen, die versicherten Summen aber um 2 71)5426 $\mathscr{N}$ zugenommen haben. Außerdem hatte eine der beiden Gesellschaften Ende 1903 noch 2420908 ä $\mathbb{N l}$ gegen 19025039 Al am Ende des Vorjahrs in Rückdeckung übernommen.

Von den selbst abgeschlossenen Versicherungen kamen

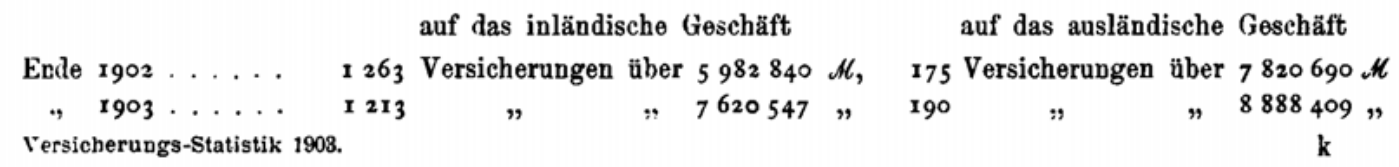


Das inländische direkte Geschäft hat also von einem Jahre zum andern um 50 Versicherungen abgenommen, die versicherte Summe ist aber um 1637707 , gewachsen, das ausländische Geschäft hat um 15 Versicherungen und 1067719 \% zugenommen. Außerordentlich groß ist der Unterschied der durchschnittlich auf eine Police versicherten Summe in inländischen und im ausländischen Geschäfte. Während dieser Betrag sich nämlich bei den deutschen Versicherungen Ende 1902 auf $4737 \mathrm{dl}$ und Ende 1903 auf 6282 .ll belief, machte er bei den ausländischen Versicherungen zu denselben Zeitpunkten 44690 und 46781 atl aus.

Die in Tab. V 14 über die Verteilung des indirekten Geschäfts auf Inland und Ausland gegebenen Zahlen weisen große Änderungen von einem Jahre zum andern auf. Diese Änderungen haben aber tatsächlich nicht in diesem Umfange stattgefunden, sie beruhen zum größten Teile darauf, daß die in Frage kommende Gesellschaft im Vorjahre nur diejenigen Versicherungen als deutsche behandelte, welche von den Erstversicherern oder, was bei weitem häufiger ist, von Rückversicherern als Versicherungen aus dem Deutschen Reiche bezeichnet waren, während sie jetzt alle von deutschen Gesellschaften in Rückdeckung übernommenen Versicherungen $\mathrm{zu}$ dem deutschen indirekten Geschäfte rechnet. Die für den Schluß der beiden Jahre eingetragenen Zahlen sind also in dieser Beziehung nicht mit einander vergleichbar.

Am Ende des Jahres r $90_{3}$ kamen von den versicherten Summen bei dem direkten Geschäfte 46,2 Prozent, bei dem indirekten dagegen 89,4 Prozent auf den inländischen, der Rest auf den ausländischen Betrieb.

Die Bruttoprämieneinnahme hat nach Tab. V 15 im Jahre rg०3 $_{3} 22408 \mathscr{M l}$ betragen, davon kommen $171811 \mathscr{H}$ oder 77,3 Prozent auf das inländische und $50597 \mathscr{H}$ oder 22,7 Prozent auf das ausländische Geschäft. Von den überhaupt entstandenen Schadensummen in Höhe von $125329 \mathrm{~d}$ kommen $80211 \mathscr{A l}$ oder 64,0 Prozent auf das Inland und $45118 \mathscr{A t}$ oder 36,0 Prozent auf das Ausland.

Von den Bruttoprämien sind ungefähr $102189 \mathscr{N}$ oder 45,9 Prozent als Rückversicherungsprämien weiter gegeben worden.

Die Sonder-Einnahmen und Ausgaben sind in Tab. V 16 zusammengestellt, sie sind bei Gruppe Il weiter verrechnet, wo die Gewinn- und Verlustrechnung und die Bilanz der hier hinsichtlich ihres Kautionsversicherungsgeschäfts behandelten Gesellschaften gegeben sind.

Die beiden ausländischen Gesellschaften hatten Ende r9o3 zusammen 2430300 , Nt. versichert, an Prämien haben sie im Berichtsjahr 31850 Al eingenommen und für Schäden 21468 .N vergütet.

\section{Kreditversicherung.}

Die Kreditversicherung ist in Jahre 1903 ebenfalls nur nebenher betrieben worden und zwar nur von der Hanseatischen See- und Allgemeinen Versicherungs - Gesellschaft und von einer ausländischen Gesellschaft. Es genügt hier, nur den Geschäftsumfang der deutschen Gesellschaft kurz zu skizzieren.

Das Geschäft ist nur direkt betrieben worden. Nach Tab. V 14 waren

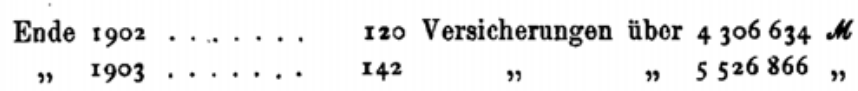

in Kraft. Es hat also die Zahl der Versicherungen um 22 und die versicherte Summe um 1220232 , $\mathbb{N}$ zugenommen.

Es kamen

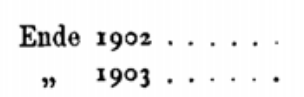

auf das inländische Geschäft

ro5 Versicherungen über 3979834 *t

$137 \quad$ " 5357066 , auf das ausländische Geschäft

15 Versicherungen über $326800 \Omega$

$5 \quad$ " 169800 , 
Der Zuwachs gebührt also sowohl was die Zahl der Versichernngen, wie auch was die Versicherungssummen betriflt, lediglich den deutschen Geschäfte, das ausländische ist zurückgegangen.

Die Bruttoprämieneinnahme hat nach Tab. V 1 õ im Jahre r9o3 308589 dl betragen, davon kommen 299548 At oder 97,1 Prozent auf das inländische und $9041 \mathrm{dl}$ oder 2,9 Prozent auf das ausländische Geschäft. Von den Schäden in einen Gesamtbetrage von $122597 \mathscr{M}$ entfallen 109693 all oder 89, a Prozent auf den inländischen und 12904 i $/$. oder 10, : Prozent auf den ausländischen Betrieb.

Von den Bruttoprämien sind angenähert 264987 \&/ oder 85,9 Prozent für Rückversicherung wieder ausgegeben worden.

Die Sonder-Einnahmen und Ausgaben sind für die Kreditversicherung in Tab. V 16 zusammengestellt, sie sind bei Gruppe IV weiter verrechnet, wo auch die Betriebsrechnung und die Bilanz der Gesellschaft gegeben sind.

\section{Maschinenversicherung.}

Die Versicherung. von Maschinen und maschinellen Vorrichtungen hat den Zweck die Schäden zu ersetzen, welche an den versicherten Gegenständen entstehen infolge von Unfällen durch den Betrieb; infolge Ungeschicklichkeit, Fahrlässigkeit oder Böswilligkeit der Arbeiter oder anderer Personen; durch Sturm, Wolkenbruch und Eisgang; infolge von Kurzschluß, oder endlich bei Montage und Demontage innerhalb des Betriebsgrundstücks.

Dieser Versicherungszweig ist im Berichtsjahre zuerst im Deutschen Reiche aufgenommen worden und zwar hat ihn in diesem Jahre nur eine Gesellschaft, die Stuttgarter Mit- und Rückversicherungs-Aktien-Gesellschaft betrieben. Es sind bisher nur direkte und nur deutsche Geschäfte abgeschlossen worden.

Am Schlusse des Jahres 1903 waren 86 Versicherungen über 8929261 , $/$ in Kraft. An Prämien wurden 20119 , / eingenommen und davon für Rückversicherung angenähert 5577 , /l oder 27,; Prozent ausgegeben. Für Schäden ohne Regulierungskosten waren $3794 . / /$ bereit zu stellen.

\section{Kaskoversicherung.}

Die Kaskoversicherung wurde im Jahre 1903 unter Reichsaufsicht nur von deutschen Unternehmungen betrieben, und zwar von 31 Gegenseitigkeitsvereinen und 4 eingetragenen Genossenschaften. Diese Gesellschaften sind zum Teil sehr klein und führen ihre Geschäfte in sehr einfachen Formen; da es nicht bei allen gelungen ist, für das Berichtsjahr befriedigende oder vergleichbare Ausweise über ihren Geschäftsbetrieb zu erhalten, mußten hier 3 Gegenseitigkeitsvereine und 1 Genossenschaft fortgelassen werden. In Vergleiche mit der vorjährigen Statistik weist die vorliegende 9 Unternehmungen mehr auf, nämlich den Schiffversicherungs-Kompakt Union zu Barßel; „Berolina“, Versicherungsverein auf Gegenseitigkeit in Charlottenburg; den Niederelbischen SchifferKompakt in Cuxhaven; die Dömitzer Flußfahrzeug-Versicherungs-Gesellschaft zu Dömitz; die Kasse zur Versicherung von Fischerfahrzeugen an der Unterweser in Geestemünde; die Schiffsversicherungs-Genossenschaft, eingetragene Genossenschaft mit unbeschränkter Haftpflicht zu Neckar-Steinach; die Kasse zur Versicherung von Fischerfahrzeugen in Ostfriesland zu Neuharlingersiel; den Kompakt „Einigkeit“ zu Oldersum und den Kompakt „Harmonie“ in Westrhauderfehn. 
Die Angaben über die Zahl und den Betrag der Versicherungen sind auch für das Berichtsjahr insofern mangelhaft, als zwar die Mehrzahl der Unternehmungen die Zahl der versicherten Fahrzeuge, einige aber statt dessen die Zahl der Mitglieder und eine Gesellschaft die Zahl der Policen mitgeteilt hat. Jedenfalls war die Zahl der versicherten Fahrzeuge Ende 1903 nicht kleiner als 6974. Die Angaben über die Versicherungssummen sind für den Schluß des Berichtsjahrs vollständig, für den Schluß des Vorjahrs fehlen sie nur bei zwei Vereinen. Nimmt man an, daß die Versicherungssumme bei diesen beiden Vereinen im Berichtsjahr ungeändert geblieben sei, so belief sich die Versicherungssumme nach Tabelle $\mathrm{V} 17$ bei 31 Unternehmungen Ende 1902 auf $42721119 \mathscr{A l}$ und Ende r 903 auf $45785568 \mathscr{M}$; sie hat also um $3064449 \mathscr{N}$ oder 7,2 Prozent zugenommen.

Die Summe der Einnahmen aller 31 Vereine hat im Jahre r903 $840452 \mathscr{M}$ betragen, die Summe der Ausgaben ist nahezu ebenso groß gewesen, nämlich $833730 \mathscr{N}$. Neunzehn Vereine haben einen Überschuß von $73714 \mathscr{H}$ erzielt und zehn einen Verlust von 66992 Nt. erlitten (Tabelle V 18).

Das Vermögen kann für den Schluß des Berichtsjahrs für einen, und für den Schluß des Vorjahrs für zwei Vereine nicht angegeben werden. Es betrug Ende $19{ }^{2}$ bei 30 Unternehmungen $1554131 \mathscr{M}$ und ist bei den 29 Vereinen, für die es für den Schluß beider Jahre bekannt ist, von $1287890 \mathscr{M}$ auf $1552430 \mathscr{M}$, also um 264540 , $\mathcal{H}$ oder 20,5 Prozent gestiegen.

Soweit Mitteilungen über die Vermögensanlagen vorhanden sind (Tabelle V 19), ergibt sich daraus, daß das Gesamtvermögen zu 23,0 Prozent in Sparkasseneinlagen, zu 47, 5 Prozent in Wertpapieren, zu 5,0 Prozent in Hypotheken, zu 5,2 Prozent in Kassenbestand und zu 19,3 Prozent in anderen Anlagen bestand. 